\title{
A fixed point theorem on noncompact manifolds
}

\author{
Peter Hochs* and Hang Wang ${ }^{\dagger}$
}

April 4, 2016

\begin{abstract}
We generalise the Atiyah-Segal-Singer fixed point theorem to noncompact manifolds. Using KK-theory, we extend the equivariant index to the noncompact setting, and obtain a fixed point formula for it. The fixed point formula is the explicit cohomological expression from Atiyah-Segal-Singer's result. In the noncompact case, however, we show in examples that this expression yields characters of infinite-dimensional representations. In one example, we realise characters of discrete series representations on the regular elements of a maximal torus, in terms of the index we define. Further results are a fixed point formula for the index pairing between equivariant K-theory and K-homology, and a non-localised expression for the index we use, in terms of deformations of principal symbols. The latter result is one of several links we find to indices of deformed symbols and operators studied by various authors.
\end{abstract}

\section{Contents}

1 Introduction 2

2 The fixed point formula 6

2.1 Localisation . . . . . . . . . . . . . . . . . . 6

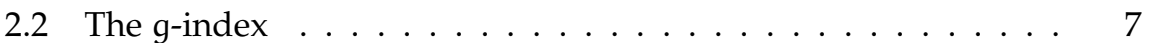

2.3 Properties of the g-index . . . . . . . . . . . . . . . 9

2.4 Fixed points . . . . . . . . . . . . . . . . . 10

2.5 The index pairing . . . . . . . . . . . . . . . 11

3 Localisation 11

3.1 Module structures . . . . . . . . . . . . . . . . . . . . . 12

3.2 Vanishing results . . . . . . . . . . . . . 13

3.3 Proofs of localisation results . . . . . . . . . . . . . . 16

${ }^{*}$ University of Adelaide, peter.hochseadelaide.edu . au

${ }^{\dagger}$ University of Adelaide, hang . wang01@adelaide.edu. au 
4 Kasparov's index theorem $\quad 17$

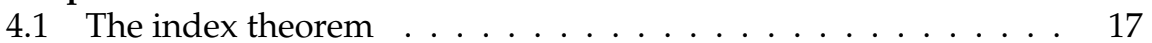

4.2 The Bott element . . . . . . . . . . . . . . . . 19

4.3 The Bott element and Dolbeault classes . . . . . . . . . . . . 20

4.4 The Atiyah-Singer index theorem . . . . . . . . . . 23

5 Proof of the fixed point formula 25

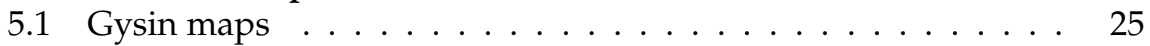

5.2 Localisation and Gysin maps .............. . . . . . . . . . . . . . .

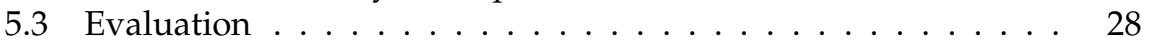

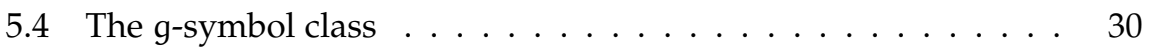

5.5 The index pairing ......................... 33

6 Examples and applications 34

6.1 Finite fixed point sets . . . . . . . . . . . . . . 34

6.2 A holomorphic linearisation theorem . . . . . . . . . . . . . 35

6.3 The circle acting on the plane ..................... 36

6.4 The circle acting on the two-sphere . . . . . . . . . . . 38

6.5 Discrete series characters . . . . . . . . . . . . . . . 39

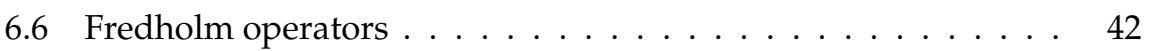

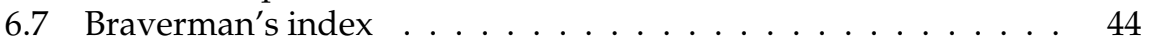

6.8 A relative index theorem . . . . . . . . . . . . . . 45

6.9 Some geometric consequences ............... 45

7 A non-localised index formula 46

\section{Introduction}

In the second part [4] of the Index of elliptic operators series, Atiyah and Segal proved a fixed point formula for compact groups and manifolds. This allows one to compute the equivariant index of an elliptic operator (or an elliptic complex) in terms of data on the fixed point sets of the group elements. In [6], a cohomological version of this formula was obtained, which we will call the Atiyah-Segal-Singer fixed point theorem. It has applications for example in representation theory. Indeed, in [2], Atiyah and Bott used a fixed point formula (which equals the Atiyah-Segal-Singer theorem in the case considered) to prove the Weyl character formula.

Our goals in this paper are to generalise the Atiyah-Segal-Singer theorem to noncompact manifolds, and to apply this generalisation in relevant situations.

\section{The main result and some applications}

We define an index on possibly noncompact manifolds, that generalises the equivariant index for compact groups and manifolds (see Definition 2.5). Assuming the fixed point set of a group element $g$ is compact, we show that this 
index is given by exactly the same cohomological expression as in the AtiyahSegal-Singer theorem. This is our main result, Theorem 2.9. We also obtain a fixed point formula for the index pairing between equivariant $\mathrm{K}$-theory and K-homology in Theorem 2.10. In the non-equivariant setting, very general expressions for this pairing were given in [17]; Theorem 2.10] is an equivariant version of these results for the operators considered here.

While the cohomological expression for the index is the same as in the compact case, in noncompact examples we see that it gives rise to characters of infinite-dimensional representations. These can never occur as indices of elliptic operators on compact manifolds, so that the theory really gives us something new. For example, we use the fixed point theorem in Subsection 6.5 to express the character of a representation in the discrete series of a semisimple Lie group in terms of our index, on the regular elements of a maximal torus. Other examples and applications are:

- a holomorphic linearisation theorem, related to [21, Chapter 4] and [12, Theorem 7.2];

- explicit computations for actions by the circle on the plane and the twosphere;

- a relation with kernels of Fredholm operators, in particular Callias-type Dirac operators [1, 14, 15, 16, 28];

- a relation with Braverman's index of deformed Dirac operators [12];

- a relative index theorem, in the spirit of [20, Theorem 4.18];

- some geometric consequences in the cases of the Hodge-Dirac and SpinDirac operators.

In all cases we consider, we find that the index can be expressed explicitly in terms of the kernel of a deformation of the operator in question. (In the discrete series example, the operator does not even have to be deformed.) On noncompact manifolds, one can often obtain a well-defined index of a Dirac operator by applying a deformation, with suitable growth behaviour. See for example [1, 12, 13, 15, 16, 24, 28, 30, 32]. This index then depends on the deformation used. While we do not use a deformation to define our index, we see in examples that it equals an index defined via a deformation. One could speculate that this means that the index we use implicitly includes a canonical choice of such a deformation. For the Callias-type operators studied in [1, 14, 15, 16, 28], their equivariant indices can be expressed as the index we define, plus a term representing the dependence on the deformation used, in terms of its behaviour "at infinity". (Previously, Callias-type operators were not studied in combination with group actions, so only non-equivariant indices were computed.)

The relation to index theory of deformed Dirac operators is strengthened in the last section of this paper, which is independent of the fixed point formula. There we give an expression for the index of elliptic operators involving deformations of their principal symbols.

Other generalised fixed point theorems include [9, Main Theorem 1] and [10, Theorem 20] (for transversally elliptic operators), [12, Theorem 7.5] (for 
deformed Dirac operators on noncompact manifolds), the results in [18] (for correspondences, generalising self-maps on manifolds), [19, Theorem 2.7] (for groupoids) and [34, Theorem 6.1] (for orbifolds).

\section{Idea of the proof}

Let us sketch some technical steps involved in defining the index and proving the fixed point formula. We consider a Riemannian manifold $M$, and an elliptic operator $\mathrm{D}$ on a vector bundle $\mathrm{E} \rightarrow \mathrm{M}$. Let $\mathrm{G}$ be a compact Lie group acting on $E$, preserving D. Under assumptions about grading and self-adjointness, we have a class $[D]$ in the equivariant $K$-homology group $K_{0}^{G}(M)$ of $M$. Let $\mathrm{g} \in \mathrm{G}$. Then we may replace $\mathrm{G}$ by the compact Abelian group generated by $\mathrm{g}$, and still retain all information about the action by the element $\mathrm{g}$. A localisation theorem in K-homology allows us to construct the g-index map

$$
\text { index }_{\mathrm{g}}: \mathrm{K}_{0}^{\mathrm{G}}(\mathrm{M}) \rightarrow \mathbb{C} \text {. }
$$

The g-index of the operator $D$ is defined as the g-index of its class [D] in $\mathrm{K}_{0}^{\mathrm{G}}(\mathrm{M})$. If $\mathrm{M}$ is compact, this is the usual equivariant index of $\mathrm{D}$, evaluated at $\mathrm{g}$.

If $M$ is compact, the principal symbol $\sigma_{D}$ of $D$ defines a class in the equivariant topological $\mathrm{K}$-theory group $\mathrm{K}_{\mathrm{G}}^{0}(\mathrm{TM})$. In our setting, $\mathrm{M}$ may be noncompact. Then we have a class

$$
\left[\sigma_{\mathrm{D}}\right] \in \mathrm{KK}_{\mathrm{G}}(\mathrm{M}, \mathrm{TM})
$$

in the equivariant KK-theory of the pair $\left(C_{0}(M), C_{0}(T M)\right)$. The DolbeaultDirac operator on TM defines a class

$$
\left[\mathrm{D}_{\mathrm{TM}}\right] \in \mathrm{KK}_{\mathrm{G}}(\mathrm{TM}, \mathrm{pt}) \text {. }
$$

An index theorem by Kasparov implies that, with respect to the Kasparov product $\otimes$ TM over $C_{0}(T M)$, we have

$$
[D]=\left[\sigma_{D}\right] \otimes_{T M}\left[D_{T M}\right] \quad \in K_{G}(M, p t)=K_{0}^{G}(M) .
$$

This generalises the Atiyah-Singer index theorem.

The proof of the fixed point formula for the $\mathrm{g}$-index of $\mathrm{D}$ is a KK-theoretic generalisation of the proof by Atiyah and Segal for the compact case in [4]. This generalisation involves Kasparov's index theorem, localisation theorems in KK-theory, and KK-theoretic versions of the Gysin wrong-way maps in Ktheory. Another ingredient is a class

$$
\sigma_{\mathrm{g}}^{\mathrm{D}} \in \mathrm{K}_{\mathrm{G}}^{0}(\mathrm{TM})_{\mathrm{g}}
$$

associated to $\sigma_{\mathrm{D}}$, in the equivariant topological K-theory of TM, localised (in the algebraic sense) at $\mathrm{g}$. Using these techniques, and keeping track of what happens in both entries in KK-theory, allows us to obtain an expression for the $g$-index of $D$ in terms of data on the fixed point set of $g$. While all constructions in the proof are KK-theoretic in nature, the end result is a purely cohomological expression. An explicit description of the class (1.1) in terms of a deformation of the symbol $\sigma_{D}$ allows us to prove a non-localised expression for the $g$-index, independent of the fixed point formula. 


\section{Outline}

The g-index is introduced in Section 2. It allows us to state the fixed point formula in Theorem 2.9

In Section 3, we prove the localisation results which imply that the g-index is well-defined. In Section 4 , we review an index theorem by Kasparov. This result, and related techniques, are used in the proof of the fixed point theorem in Section 5

The applications and examples mentioned above are discussed in Section 6 In Section 7 we obtain a non-localised expression for the $g$-index of an elliptic operator.

\section{Acknowledgements}

The authors are grateful to Gert Heckman, Bram Mesland, Yanli Song and Michèle Vergne for helpful discussions and comments. Special thanks go to Gennadi Kasparov for his explanations of the material in Section 4 This first author was supported by the European Union, through Marie Curie fellowship PIOF-GA-2011-299300.

\section{Notation}

If $A$ is a subset of a set $B$, then we will denote the inclusion map $A \hookrightarrow B$ by $j_{A}^{B}$. We denote the one-point set by pt. For any set $A$, we will write $p^{A}$ for the map from $A$ to pt.

If $U$ is an open subset of a locally compact Hausdorff space $X$, then we denote by $k_{\mathrm{U}}^{X}$ the inclusion map $\mathrm{C}_{0}(\mathrm{U}) \hookrightarrow \mathrm{C}_{0}(\mathrm{X})$ defined by extending functions by zero outside $U$. If $Y$ is another locally compact Hausdorff space, we will write

$$
\mathrm{KK}(\mathrm{X}, \mathrm{Y}):=\mathrm{KK}\left(\mathrm{C}_{0}(\mathrm{X}), \mathrm{C}_{0}(\mathrm{Y})\right)
$$

and similarly for equivariant KK-theory. The Kasparov product $\otimes_{C_{0}(X)}$ over $C_{0}(X)$ will also be denoted by $\otimes x$. If $X$ has a Borel measure, and $E \rightarrow X$ is a Hermitian vector bundle, then the $*$-homomorphism $\pi_{X}: C_{0}(X) \rightarrow \mathcal{B}\left(L^{2}(E)\right)$ is given by the pointwise multiplication on $\mathrm{L}^{2}$-sections of $\mathrm{E}$. If $\mathrm{G}$ is a locally compact group acting on $X$, and $H<G$ is a subgroup, we will write $G \times_{H} X$ for the quotient of $\mathrm{G} \times \mathrm{X}$ by the action by $\mathrm{H}$ given by

$$
h \cdot(g, x)=\left(g h^{-1}, h x\right),
$$

for $h \in H, g \in G$ and $x \in X$.

If $M$ is a manifold, its tangent bundle projection $T M \rightarrow M$ is denoted by $\tau_{M}$. If a Riemannian metric is given, we will often tacitly use it to identify the tangent bundle of $M$ with the cotangent bundle. The complexification of a vector space or vector bundle is denoted by a subscript $\mathbb{C}$. 


\section{The fixed point formula}

Our goal in this paper is to generalise the Atiyah-Segal-Singer fixed point theorem ([6. Theorem 3.9], based on [4, Theorem 2.12]) to noncompact manifolds, and to find interesting applications of this generalisation. This leads us to define the g-index in Subsection 2.2. The key to defining the g-index is a localisation theorem, which is stated in Subsection 2.1. The main result of this paper is the fixed point formula in Theorem 2.9, stated in Subsection 2.4 This formula is entirely cohomological, and does not involve KK-theory. Some properties of the g-index are given in Subsection 2.3.

Throughout this paper, $M$ will be a Riemannian manifold. We consider an isometric diffeomorphism $g$ from $M$ to itself. Suppose the closure of the powers of $g$ in the isometry group (with respect to the compact-open topology) is a compact group G. Equivalently, suppose $g$ is an element of a compact group $H$ acting isometrically on $M$, and let $G<H$ be the closed subgroup generated by $\mathrm{g}$. In any case, $\mathrm{G}$ is Abelian. Let $\mathrm{M}^{\mathrm{g}}$ be the fixed point set of $\mathrm{g}$.

Let $\mathrm{E}=\mathrm{E}^{+} \oplus \mathrm{E}^{-} \rightarrow \mathrm{M}$ be a $\mathbb{Z}_{2}$-graded, Hermitian vector bundle. Let $\mathrm{D}$ be an odd, essentially self-adjoint, elliptic differential operator, with principal symbol $\sigma_{\mathrm{D}}$. (For example, D can be a Dirac-type operator on a complete manifold.) We will also write $\mathrm{D}$ for the self-adjoint closure of $\mathrm{D}$. Then we have the element

$$
[D]:=\left[\mathrm{L}^{2}(\mathrm{E}), \frac{\mathrm{D}}{\sqrt{\mathrm{D}^{2}+1}}, \pi_{M}\right]
$$

of the equivariant $\mathrm{K}$-homology group $\mathrm{KK}_{\mathrm{G}}(\mathrm{M}, \mathrm{pt}):=\mathrm{KK}_{\mathrm{G}}\left(\mathrm{C}_{0}(\mathrm{M}), \mathbb{C}\right)$. Here $\pi_{M}: C_{0}(M) \rightarrow \mathcal{B}\left(L^{2}(E)\right)$ is given by pointwise multiplication. For background material on KK-theory, see [11, Chapter VIII].

\subsection{Localisation}

Let $R(G)_{g}:=R(G)_{I_{g}}$ be the localisation of the representation ring $R(G)$ at the prime ideal

$$
I_{g}:=\{\chi \in R(G) ; \chi(g)=0\} .
$$

For any module $\mathcal{M}$ over $R(G)$, we write $\mathcal{M}_{g}:=\mathcal{M}_{I_{g}}$ for the corresponding localised module over $R(G)_{g}$. Similarly, if $m \in \mathcal{M}$, and $\varphi: \mathcal{M} \rightarrow \mathcal{M}^{\prime}$ is a module homomorphism to another such module, we write $m_{g} \in \mathcal{M}_{g}$ and

$$
\varphi_{g}: \mathcal{M}_{g} \rightarrow \mathcal{M}_{g}^{\prime}
$$

for the corresponding localised versions.

For any two $G-C^{*}$ algebras $A$ and $B$, the group $K_{K}(A, B)$ is a module over the ring $R(G)=K K_{G}(\mathbb{C}, \mathbb{C})$, via the exterior Kasparov product. Fix a G-C* algebra $A$. The inclusion map

$$
\mathrm{j}_{M^{g}}^{M}: M^{g} \hookrightarrow M
$$

induces

$$
\left(j_{M^{g}}^{M}\right)_{g}^{*}: K_{G}\left(A, C_{0}(M)\right)_{g} \rightarrow K^{G}\left(A, C_{0}\left(M^{g}\right)\right)_{g} .
$$


Theorem 2.1. If $\mathrm{A}$ is separable, the map $\left(\mathrm{j}_{\mathrm{Mg}}^{\mathrm{M}}\right)_{\mathrm{g}}^{*}$ is an isomorphism of Abelian groups. This is still true if $M \backslash M^{9}$ is a manifold, rather than all of $M$.

Remark 2.2. If $A=\mathbb{C}$, then this reduces to [4, Theorem 1.1]. We need this more general statement, because in the noncompact case, principal symbols define classes in $\mathrm{KK}_{\mathrm{G}}\left(\mathrm{C}_{0}(\mathrm{M}), \mathrm{C}_{0}(\mathrm{TM})\right)$ as in (4.3), rather than in $\mathrm{KK}_{\mathrm{G}}\left(\mathbb{C}, \mathrm{C}_{0}(\mathrm{TM})\right.$ ) when $M$ is compact.

We will also use an analogue of Theorem 2.1 for the first entry in KK-theory. Its formulation is slightly more subtle.

Theorem 2.3. Suppose that $\mathrm{M}^{\mathrm{g}}$ is compact and that $\mathrm{A}$ is $\sigma$-unital. Let $\mathrm{U}, \mathrm{V} \subset \mathrm{M}$ be two G-invariant, relatively compact open neighbourhoods of $\mathrm{M}^{\mathrm{g}}$, such that $\overline{\mathrm{U}} \subset \mathrm{V}$. Then the map

$$
\left(\left(j \frac{\mathrm{V}}{\mathrm{U}}\right)_{*}\right)_{\mathrm{g}}: \mathrm{KK}_{\mathrm{G}}\left(\mathrm{C}_{0}(\overline{\mathrm{U}}), \mathrm{A}\right)_{\mathrm{g}} \rightarrow \mathrm{KK}_{\mathrm{G}}\left(\mathrm{C}_{0}(\mathrm{~V}), \mathrm{A}\right)_{\mathrm{g}}
$$

is an isomorphism of Abelian groups. This is still true if $\mathrm{M} \backslash \mathrm{M}^{\mathrm{g}}$ is a manifold, rather than all of $M$.

Theorems 2.1 and 2.3 will be proved in Section 3, for graded KK-theory, i.e. the combination of even and odd KK-theory. We will only apply the even versions, however. The cases where only $M \backslash M^{g}$ is a manifold were included because we will also apply Theorem 2.3 to one-point compactifications of manifolds.

\subsection{The g-index}

Suppose the fixed point set $\mathrm{M}^{\mathrm{g}}$ is compact. Let $\mathrm{U}, \mathrm{V}$ be as in Theorem 2.3 Consider the proper map $p^{\bar{u}}: \bar{U} \rightarrow p t$, and the inclusion map $k_{V}^{M}: C_{0}(V) \rightarrow$ $C_{0}(M)$ given by extending function by zero outside $V$. Let $A$ be a $\sigma$-unital $\mathrm{G}-\mathrm{C}^{*}$ algebra. By Theorem 2.3, we have the maps

$$
\begin{array}{r}
\mathrm{KK}_{\mathrm{G}}\left(\mathrm{C}_{0}(\mathrm{M}), A\right)_{\mathrm{g}} \stackrel{\left(\mathrm{K}_{\mathrm{V}}^{\mathrm{M}}\right)_{g}^{*}}{\longrightarrow} \mathrm{KK}_{\mathrm{G}}\left(\mathrm{C}_{0}(\mathrm{~V}), A\right)_{\mathrm{g}} \stackrel{\left(\left(j \frac{\mathrm{V}}{\mathrm{U}}\right)_{*}\right)_{g}^{-1}}{\longrightarrow} \mathrm{KK}_{\mathrm{G}}(\mathrm{C}(\overline{\mathrm{U}}), A)_{\mathrm{g}} \\
\stackrel{\left(\mathrm{p}_{*}^{\overline{\mathrm{u}}}\right)_{g}}{\longrightarrow} \mathrm{KK}_{\mathrm{G}}(\mathbb{C}, A)_{g} .
\end{array}
$$

Lemma 2.4. The composition (2.2) is independent of the sets $\mathrm{U}$ and $\mathrm{V}$.

Proof. To prove independence of $\mathrm{U}$, let $\mathrm{U}^{\prime}$ be a G-invariant, relatively compact neighbourhood of $M^{g}$ such that $\overline{\mathrm{U}^{\prime}} \subset \mathrm{U}$. Then we have the commutative diagram

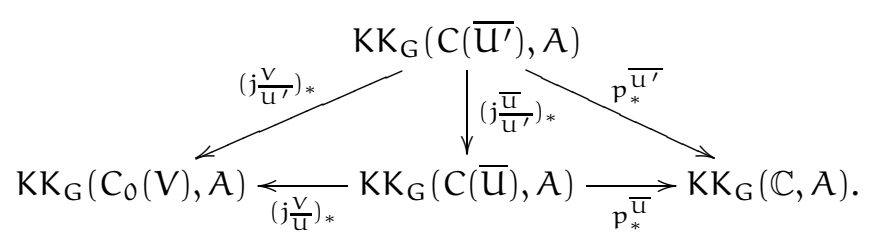


Commutativity of this diagram implies that

$$
\left(p_{*}^{\overline{u^{\prime}}}\right)_{g} \circ\left(\left(j \frac{V}{\mathrm{U}^{\prime}}\right)_{*}\right)_{g}^{-1}=\left(p_{*}^{\bar{u}}\right)_{g} \circ\left(\left(j \frac{V}{\mathrm{U}}\right)_{*}\right)_{g}^{-1} .
$$

So (2.2) is indeed independent of $U$.

To prove independence of $\mathrm{V}$, let $\mathrm{V}^{\prime}$ be a $\mathrm{G}$-invariant, relatively compact open subset of $M$ containing $V$. Then the following diagram commutes:

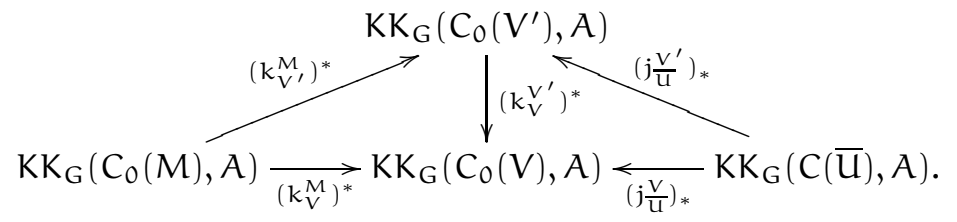

Therefore, we have

$$
\left(\left(j \frac{\mathrm{V}^{\prime}}{\mathrm{U}}\right)_{*}\right)_{\mathrm{g}}^{-1} \circ\left(\mathrm{k}_{\mathrm{V}^{\prime}}^{\mathrm{M}}\right)_{\mathrm{g}}^{*}=\left(\left(\mathrm{j} \frac{\mathrm{V}}{\mathrm{U}}\right)_{*}\right)_{\mathrm{g}}^{-1} \circ\left(\mathrm{k}_{\mathrm{V}}^{\mathrm{M}}\right)_{\mathrm{g}}^{*}
$$

so that (2.2) is independent of $\mathrm{V}$.

To define the g-index, we only need the case of Lemma 2.4 where $A=\mathbb{C}$. Later we will also use the general case, however.

Let

$$
\mathrm{ev}_{\mathrm{g}}: \mathrm{R}(\mathrm{G}) \rightarrow \mathbb{C}
$$

be defined by evaluating characters at $g$, i.e. $\operatorname{ev}_{g}(\chi):=\chi(g)$, for $\chi \in R(G)$. In view of Lemma 2.4, we obtain a well-defined index as follows.

Definition 2.5. The g-index is the map

$$
\text { index }_{\mathrm{g}}: \mathrm{KK}_{\mathrm{G}}(\mathrm{M}, \mathrm{pt}) \rightarrow \mathbb{C}
$$

defined as the composition

$\mathrm{KK}_{\mathrm{G}}(\mathrm{M}, \mathrm{pt}) \hookrightarrow \mathrm{KK}_{\mathrm{G}}(\mathrm{M}, \mathrm{pt})_{\mathrm{g}} \stackrel{\left(\mathrm{p}_{*}^{\overline{\mathrm{T}}}\right)_{\mathrm{g}} \circ\left(\left(j_{\mathrm{U}}^{\mathrm{V}}\right)_{*}\right)_{\mathrm{g}}^{-1} \circ\left(\mathrm{K}_{\mathrm{V}}^{\mathrm{M}}\right)_{\mathrm{g}}^{*}}{\longrightarrow} \mathrm{KK} \mathrm{K}_{\mathrm{G}}(\mathrm{pt}, \mathrm{pt})_{\mathrm{g}} \cong \mathrm{R}(\mathrm{G})_{\mathrm{g}} \stackrel{\left(\mathrm{ev}_{\mathrm{g}}\right)_{\mathrm{g}}}{\longrightarrow} \mathbb{C}$.

We will write

$$
\operatorname{index}_{g}(D):=\operatorname{index}_{g}[D]
$$

where $[D] \in K_{K_{G}}(M, p t)$ is the class (2.1).

Note that $\left(k_{V}^{M}\right)_{g}^{*}[D]_{g}$ is simply the localisation at $g$ of the $K$-homology class of the restriction of $\mathrm{D}$ to $\mathrm{V}$.

Remark 2.6. The g-index of D could also have been called the D-Lefschetz number of $g$. 


\subsection{Properties of the $g$-index}

If $M$ is compact, then we may take $U=V=M$ in Definition 2.5. Furthermore, the map $p^{M}: M \rightarrow p t$ is proper. In that case, the composition (2.2) simply equals the map

$$
\left(\mathrm{p}_{*}^{M}\right)_{\mathrm{g}}: \mathrm{KK}_{\mathrm{G}}\left(\mathrm{C}_{\mathrm{O}}(\mathrm{M}), A\right)_{\mathrm{g}} \rightarrow \mathrm{KK}_{\mathrm{G}}(\mathbb{C}, A)_{\mathrm{g}} .
$$

If $A=\mathbb{C}$, then it follows that for compact $M$, the $g$-index of $D$ equals

$$
\operatorname{index}_{g}(D)=\operatorname{index}_{G}(D)(g) \text {, }
$$

the usual equivariant index of $\mathrm{D}$ evaluated at $\mathrm{g}$. Note that on the right hand side of (2.4), G can be any compact Lie group acting isometrically on $M$, if the action lifts to $E$, commutes with $\mathrm{D}$, and contains $\mathrm{g}$.

In general, however, the g-indices on noncompact manifolds give us something more general than the equivariant index in the compact case. In the examples in Section 6, we will see that the g-index can be used to describe characters of infinite-dimensional representations. These cannot be realised as indices on compact manifolds. And even on compact manifolds, an equivariant index can be decomposed into $\mathrm{g}$-indices which individually correspond to infinite-dimensional representations. See Subsection 6.4

The $\mathrm{g}$-index has an excision property.

Lemma 2.7. Let $\mathrm{V}$ be a G-invariant, relatively compact, open neighbourhood of $\mathrm{M}^{\mathrm{g}}$. Suppose there is a $\mathrm{G}$-equivariant open embedding $\mathrm{V} \hookrightarrow \tilde{\mathrm{M}}$ into a $\mathrm{G}$-manifold $\tilde{\mathrm{M}}$. Suppose the action by $\mathrm{G}$ on $\tilde{\mathrm{M}}$ has no fixed points outside $\mathrm{V}$. Suppose there is a Hermitian, $\mathbb{Z}_{2}$-graded G-vector bundle $\tilde{\mathrm{E}} \rightarrow \tilde{\mathrm{M}}$ and an odd, self-adjoint, elliptic differential operator $\tilde{\mathrm{D}}$ on $\tilde{\mathrm{E}}$ such that $\left.\tilde{\mathrm{E}}\right|_{V}=\left.\mathrm{E}\right|_{V}$ and $\left.\tilde{\mathrm{D}}\right|_{V}=\left.\mathrm{D}\right|_{V}$. Then

$$
\operatorname{index}_{g}(D)=\operatorname{index}_{g}(\tilde{D}) \text {. }
$$

Proof. By Proposition 10.8.8 in [23], we have

$$
\left(k_{V}^{M}\right)^{*}[D]=\left(k_{V}^{\tilde{M}}\right)^{*}[\tilde{D}] \in K_{G}(V, p t) .
$$

This implies the claim.

Example 2.8. Suppose $M$ has a G-equivariant Spin-structure, and let $D$ be the Spin-Dirac operator. Let $M \hookrightarrow \tilde{M}$ be a G-equivariant open embeddng into a compact G-manifold $\tilde{M}$ with a G-equivariant Spin-structure. If $G$ is connected and $\operatorname{index}_{g}(D) \neq 0$, then $g$ must have a fixed point in $\tilde{M} \backslash M$. Indeed, Atiyah and Hirzebruch [3] showed that the $g$-index of the Spin-Dirac operator on $\tilde{M}$ is zero in this case. So the claim follows by Lemma 2.7

Another property of the $g$-index is multiplicativity. Let $D_{1}$ and $D_{2}$ be operators like $D$ on manifolds $M_{1}$ and $M_{2}$ respectively, and consider the product operator

$$
\mathrm{D}_{1} \times \mathrm{D}_{2}:=\mathrm{D}_{1} \otimes 1+1 \otimes \mathrm{D}_{2}
$$


on $M_{1} \times M_{2}$ (where graded tensor products are used). Then functoriality of the Kasparov product implies that

$$
\operatorname{index}_{g}\left(D_{1} \times D_{2}\right)=\operatorname{index}_{g}\left(D_{1}\right) \operatorname{index}_{g}\left(D_{2}\right) .
$$

In the index theory of deformed Dirac operators developed in [12], the deformation used means that an analogous multiplicativity property is highly nontrivial, see [25, 30, 32].

\subsection{Fixed points}

Having defined the g-index, we can state the main result of this paper. We will use the fact that the connected components of the fixed point set $M^{g}$ are smooth submanifolds of $M$, possibly of different dimensions.

Since $M^{g}$ is compact, the restriction to $\mathrm{TM}^{\mathrm{g}}$ of the principal symbol $\sigma_{\mathrm{D}}$ of $D$ defines a class

$$
\left[\left.\sigma_{\mathrm{D}}\right|_{\mathrm{TM}} \mathrm{g}\right] \in \mathrm{KK}_{\mathrm{G}}\left(\mathrm{pt}, \mathrm{TM}^{\mathrm{g}}\right)
$$

Let $\mathrm{N} \rightarrow \mathrm{M}^{\mathrm{g}}$ be the union of the normal bundles to each of the components of $M^{9}$. Consider the topological K-theory class

$$
\left[\wedge \mathrm{N}_{\mathbb{C}}\right]:=\left[\bigoplus_{j} \wedge^{2 j} \mathrm{~N} \otimes \mathbb{C}\right]-\left[\bigoplus_{j} \wedge^{2 j+1} \mathrm{~N} \otimes \mathbb{C}\right] \in \mathrm{KK}_{\mathrm{G}}\left(\mathrm{pt}, \mathrm{M}^{\mathrm{g}}\right)
$$

For any trivial G-space $X$, we have

$$
\mathrm{KK}_{\mathrm{G}}(\mathrm{pt}, X) \cong \mathrm{KK}(\mathrm{pt}, \mathrm{X}) \otimes \mathrm{R}(\mathrm{G}) .
$$

We can evaluate the factor in $R(G)$ of any class $a \in K K_{G}(p t, X)$ at $g$, to obtain $\mathrm{a}(\mathrm{g}) \in \mathrm{KK}(\mathrm{pt}, \mathrm{X}) \otimes \mathbb{C}$. In this way, evaluating the classes (2.5) and (2.6) at $\mathrm{g}$ gives the classes

$$
\left[\left.\sigma_{\mathrm{D}}\right|_{\mathrm{TM}} \mathrm{g}\right](\mathrm{g}) \in \mathrm{KK}\left(\mathrm{pt}, \mathrm{TM}^{\mathrm{g}}\right) \otimes \mathbb{C}
$$

and

$$
\left[\wedge N_{\mathbb{C}}\right](\mathrm{g}) \in \mathrm{KK}\left(\mathrm{pt}, \mathrm{M}^{\mathrm{g}}\right) \otimes \mathbb{C}
$$

respectively.

Consider the Chern characters

$$
\begin{aligned}
& \text { ch: } \mathrm{KK}\left(\mathrm{pt}, \mathrm{TM}^{\mathrm{g}}\right) \rightarrow \mathrm{H}^{*}\left(\mathrm{TM}^{\mathrm{g}}\right) ; \\
& \text { ch: } \mathrm{KK}\left(\mathrm{pt}, \mathrm{M}^{\mathrm{g}}\right) \rightarrow \mathrm{H}^{*}\left(\mathrm{M}^{\mathrm{g}}\right),
\end{aligned}
$$

defined on each smooth component of $\mathrm{M}^{\mathrm{g}}$ separately. By [4. Lemma 2.7], the K-theory class (2.7) is invertible, hence so is its Chern character. An explicit expression for the inverse

$$
\frac{1}{\operatorname{ch}\left(\left[\bigwedge N_{\mathbb{C}}\right](g)\right)} \in H^{*}\left(M^{g}\right) \otimes \mathbb{C}
$$

of this element is given in [6, eq. (3.8)]. The cohomology group $\mathrm{H}^{*}\left(\mathrm{M}^{9}\right)$ acts on $\mathrm{H}^{*}\left(\mathrm{TM}^{9}\right)$ via the pullback along the tangent bundle projection $\tau_{\mathrm{M}^{g}}$. Let $\operatorname{Todd}\left(\mathrm{TM}^{\mathrm{g}} \otimes \mathbb{C}\right)$ be the cohomology class on $\mathrm{M}^{\mathrm{g}}$ obtained by putting together the Todd-classes of the complexified tangent bundles to all components of $\mathrm{M}^{\mathrm{g}}$. 
Theorem 2.9 (Fixed point formula). The g-index of $\mathrm{D}$ equals

$$
\operatorname{index}_{g}(D)=\int_{\mathrm{TM}^{g}} \frac{\operatorname{ch}\left(\left[\left.\sigma_{\mathrm{D}}\right|_{\mathrm{TM}_{\mathrm{g}}}\right](\mathrm{g})\right) \operatorname{Todd}\left(\mathrm{TM}^{\mathrm{g}} \otimes \mathbb{C}\right)}{\operatorname{ch}\left(\left[\bigwedge \mathrm{N}_{\mathbb{C}}\right](\mathrm{g})\right)} .
$$

The integral in this expression is the sum of the integrals over all connected components of $\mathrm{TM}^{\mathrm{g}}$ of the integrand corresponding to each component.

If $M$ is compact, then (2.4) implies that Theorem 2.9 reduces to the AtiyahSegal-Singer fixed point formula [6, Theorem 3.9].

\subsection{The index pairing}

In the course of the proof of Theorem 2.9 , we will also find a fixed point formula for the index pairing (i.e. the Kasparov product)

$$
\mathrm{KK}_{\mathrm{G}}(\mathrm{pt}, \mathrm{M}) \times \mathrm{KK}_{\mathrm{G}}(\mathrm{M}, \mathrm{pt}) \rightarrow \mathrm{KK}_{\mathrm{G}}(\mathrm{pt}, \mathrm{pt}) .
$$

Note that any element of the equivariant topological $\mathrm{K}$-theory group $\mathrm{KK}_{\mathrm{G}}(\mathrm{pt}, \mathrm{M})$ can be represented by a formal difference $\left[\mathrm{F}_{0}\right]-\left[\mathrm{F}_{1}\right]$, for two G-equivariant vector bundles $F_{0}, F_{1} \rightarrow M$ that are equal outside a compact set. We will write $F:=F_{0} \oplus F_{1}$, with the $\mathbb{Z}_{2}$-grading where $F_{0}$ is the even part and $F_{1}$ the odd part, and $[\mathrm{F}]:=\left[\mathrm{F}_{0}\right]-\left[\mathrm{F}_{1}\right] \in \mathrm{KK}_{\mathrm{G}}(\mathrm{pt}, \mathrm{M})$.

Theorem 2.10 (Fixed point formula for the index pairing). We have

$$
\left([\mathrm{F}] \otimes_{\mathrm{M}}[\mathrm{D}]\right)(\mathrm{g})=\int_{\mathrm{TM}^{g}} \frac{\operatorname{ch}\left(\left[\left.\mathrm{F}\right|_{\mathrm{M}^{g}}\right](\mathrm{g})\right) \operatorname{ch}\left(\left[\left.\sigma_{\mathrm{D}}\right|_{\mathrm{TM}^{g}}\right](\mathrm{g})\right) \operatorname{Todd}\left(\mathrm{TM}^{\mathrm{g}} \otimes \mathbb{C}\right)}{\operatorname{ch}\left(\left[\wedge \mathrm{N}_{\mathbb{C}}\right](\mathrm{g})\right)} .
$$

Recall that $M^{g}$ was assumed to be compact, and that we use the action by the cohomology of $\mathrm{M}^{\mathrm{g}}$ on the cohomology of $\mathrm{TM}^{\mathrm{g}}$ via the pullback along $\tau_{\mathrm{M}^{g}}$.

Theorem 3.33 in [17] is a non-equivariant index formula for the index pairing in a more general context. Theorem 2.10 is an equivariant version of this result, for operators like $D$.

The proof of Theorem 2.10 is simpler than that of Theorem 2.9. because it does not involve localisation in the first entry of KK-theory. Theorem 2.9 is needed for the examples and applications in Section 6, such as the relation with characters of dicrete series representations. The reason for this is that Theorem 2.9 provides an expression for an index of the operator D itself, without the need to twist it by a K-theory class.

\section{Localisation}

We now turn to a proof of Theorems 2.1 and 2.3. This involves certain module structures discussed in Subsection 3.1, which are used to prove vanishing results in Subsection 3.2

In this section, we will consider graded KK-theory, i.e. the direct sum of even and odd KK-theory. 


\subsection{Module structures}

In this subsection only, let $\mathrm{G}$ be any locally compact group.

Proposition 3.1. Let $\mathrm{H}<\mathrm{G}$ be a compact subgroup. Let $\mathrm{Y}$ be a locally compact, Hausdorff, proper G-space for which there is an equivariant, continuous map $\mathrm{Y} \rightarrow$ $\mathrm{G} / \mathrm{H}$. Then for any $\mathrm{G}-\mathrm{C}^{*}$-algebra $\mathrm{A}$, the groups

$$
\mathrm{KK}_{\mathrm{G}}\left(\mathrm{A}, \mathrm{C}_{\mathrm{O}}(\mathrm{Y})\right) \text { and } \mathrm{KK}_{\mathrm{G}}\left(\mathrm{C}_{0}(\mathrm{Y}), \mathrm{A}\right)
$$

have structures of a unital $\mathrm{R}(\mathrm{H})$-modules.

Proposition 3.1 follows from the fact that vector bundles, even on noncompact spaces, define classes in KK-theory in the following way. This is probably well-known, but we include a proof for completeness' sake.

Let $X$ be a locally compact Hausdorff space, on which a locally compact group $\mathrm{G}$ acts properly. Let $\mathrm{E} \rightarrow \mathrm{X}$ be a Hermitian $\mathrm{G}$-vector bundle. The space $\Gamma_{0}(E)$ of continuous sections of $E$ vanishing at infinity is a right Hilbert $C_{0}(X)-$ module by pointwise multiplication and inner products. Let $\pi_{X}: C_{0}(X) \rightarrow$ $\mathcal{B}\left(\Gamma_{0}(E)\right)$ be given by pointwise multiplication.

Lemma 3.2. The triple

$$
\left(\Gamma_{0}(E), 0, \pi_{X}\right)
$$

is a $\mathrm{G}$-equivariant Kasparov $\left(\mathrm{C}_{0}(\mathrm{X}), \mathrm{C}_{0}(\mathrm{X})\right.$ )-cycle.

We will denote the class in $\mathrm{KK}_{\mathrm{G}}(\mathrm{X}, \mathrm{X})$ defined by (3.1) by $[\mathrm{E}]$.

Proof. We will show that for all $f \in C_{0}(X)$, the operator $\pi_{X}(f)$ on $\Gamma_{0}(E)$ is compact. This implies the claim.

Let $\mathrm{U} \subset \mathrm{X}$ be a relatively compact open subset admitting an orthonormal frame $\left\{e_{1}, \ldots, e_{r}\right\}$ of $\left.E\right|_{u}$. Let $s \in \Gamma_{0}(E)$. Then

$$
\left.s\right|_{\mathrm{u}}=\sum_{j=1}^{\mathrm{r}}\left(e_{j}, s\right)_{\mathrm{E}} e_{j} .
$$

Here $(-,-)_{E}$ is the metric on $E$. So if $f \in C_{0}(X)$ is supported inside $U$, then

$$
\pi_{X}(f) s=\sum_{j=1}^{r}\left(e_{j}, f s\right) e_{j}=\sum_{j=1}^{r}\left(\bar{f} e_{j}, s\right) e_{j} .
$$

By extending the sections $e_{j}$ outside $U$ to elements of $\Gamma_{0}(E)$, we find that $\pi_{X}(f)$ is a finite-rank operator.

For a general $f \in C_{c}(X)$, there is a finite open cover $\left\{U_{j}\right\}_{j=1}^{n}$ of $\operatorname{supp}(f)$ such that every set $U_{j}$ admits a local orthonormal frame for $E$. Let $\left\{\varphi_{j}\right\}_{j=1}^{n}$ be functions such that $\operatorname{supp}\left(\varphi_{j}\right) \subset \mathrm{U}_{j}$, and $\sum_{j=1}^{n} \varphi_{j}$ equals one on $\operatorname{supp}(f)$. Then, by the preceding argument,

$$
\pi_{X}(f)=\sum_{j=1}^{n} \pi_{X}\left(\varphi_{j} f\right)
$$

is a finite-rank operator. Hence for all $f \in C_{0}(X)$, the operator $\pi_{X}(f)$ on $\Gamma_{0}(E)$ is indeed compact. 
Now consider the situation of Proposition 3.1 Let $p: Y \rightarrow G / H$ be an equivariant, continuous map. Let $\mathrm{V}$ be a finite-dimensional representation space of $\mathrm{H}$. We have the $\mathrm{G}$-vector bundles

$$
\mathrm{G} \times{ }_{H} \mathrm{~V} \rightarrow \mathrm{G} / \mathrm{H}
$$

and

$$
E_{V}:=p^{*}\left(G \times_{H} V\right) \rightarrow Y .
$$

By Lemma 3.2, this vector bundle defines a class

$$
\left[\mathrm{E}_{\mathrm{V}}\right] \in \mathrm{KK}_{\mathrm{G}}(\mathrm{Y}, \mathrm{Y}) .
$$

Lemma 3.3. The map from $\mathrm{R}(\mathrm{H})$ to $\mathrm{KK}_{\mathrm{G}}(\mathrm{Y}, \mathrm{Y})$ given by

$$
[\mathrm{V}] \mapsto\left[\mathrm{E}_{\mathrm{V}}\right]
$$

as above, is a ring homomorphism.

Proof. This follows from the fact that, in the setting of Lemma 3.2, for any two Hermitian G-vector bundles $E, E^{\prime} \rightarrow X$, one has

$$
[E] \otimes X\left[E^{\prime}\right]=\left[E \otimes E^{\prime}\right] .
$$

The ring homomorphism of Lemma 3.3 defines the module structures sought in Proposition 3.1. which has therefore been proved. If $A=\mathbb{C}$ and $Y$ is compact, the $R(H)$-module structure on $\mathrm{KK}_{\mathrm{G}}\left(\mathbb{C}, \mathrm{C}_{0}(\mathrm{Y})\right)$ defined in this way is the one used in [4].

\subsection{Vanishing results}

We will prove Theorems 2.1 and 2.3 by generalising Atiyah and Segal's proof of [4, Theorem 1.1]. An important step is the following generalisation of [4, Corollary 1.4].

Proposition 3.4. Let $\mathrm{H}<\mathrm{G}$ be a closed subgroup such that $\mathrm{g} \notin \mathrm{H}$. Let $\mathrm{Y}$ be a compact G-space for which there is an equivariant map $\mathrm{Y} \rightarrow \mathrm{G} / \mathrm{H}$ and $\mathrm{A}$ a G-C*algebra. Then

$$
\mathrm{KK}_{\mathrm{G}}\left(\mathrm{A}, \mathrm{C}_{0}(\mathrm{Y})\right)_{\mathrm{g}}=\mathrm{KK}_{\mathrm{G}}\left(\mathrm{C}_{0}(\mathrm{Y}), \mathrm{A}\right)_{\mathrm{g}}=0 .
$$

Proof. By [4, Corollary 1.3], we have $\mathrm{R}(\mathrm{H})_{\mathrm{g}}=0$. As Atiyah and Segal argued below that corollary, it is therefore enough to show that $\mathrm{KK}_{\mathrm{G}}\left(\mathrm{A}, \mathrm{C}_{0}(\mathrm{Y})\right)$ and $\mathrm{KK}_{\mathrm{G}}\left(\mathrm{C}_{0}(\mathrm{Y}), \mathrm{A}\right)$ are unital $\mathrm{R}(\mathrm{H})$-modules. Hence the claim follows from Proposition 3.1 .

We will deduce Theorems 2.1 and 2.3 from the following special cases. 
Proposition 3.5. In the setting of Theorem 2.1] suppose $\mathrm{g}$ has no fixed points in $\mathrm{M}$. Then, if A is separable, we have

$$
\mathrm{KK}_{\mathrm{G}}\left(\mathrm{A}, \mathrm{C}_{\mathrm{O}}(\mathrm{M})\right)_{\mathrm{g}}=0 .
$$

If $\mathrm{A}$ is $\sigma$-unital, then for all $\mathrm{G}$-invariant, relatively compact open subsets $\mathrm{U} \subset \mathrm{M}$,

$$
\mathrm{KK}_{\mathrm{G}}\left(\mathrm{C}_{0}(\mathrm{U}), A\right)_{\mathrm{g}}=0 \text {. }
$$

If $A=\mathbb{C}$, then (3.2) is precisely [4. Proposition 1.5]. By a generalisation of the arguments in [4, Section 1], we will deduce Proposition 3.5] from Proposition 3.4

By Palais' slice theorem [31, Proposition 2.2.2], there is an open $\operatorname{cover}\left\{\mathrm{U}_{j}\right\}_{j=1}^{\infty}$ of $M$ by $G$-invariant open sets such that for all $j$,

$$
\overline{\mathrm{U}_{j}} \cong \mathrm{G} \times_{\mathrm{H}_{j}} \overline{\mathrm{S}_{\mathrm{j}}}
$$

(via the action map), for the stabiliser $\mathrm{H}_{j}<\mathrm{G}$ of a point in $\mathrm{U}_{j}$, and an $\mathrm{H}_{j}$ invariant submanifold $S_{j} \subset M$. Suppose that $g$ has no fixed points. Then it does not lie in any of the stabilisers $\mathrm{H}_{j}$. Therefore, Proposition 3.4 implies that

$$
\mathrm{KK}_{\mathrm{G}}\left(A, \mathrm{C}_{0}\left(\overline{\mathrm{U}_{j}}\right)\right)_{\mathrm{g}}=\mathrm{KK}_{\mathrm{G}}\left(\mathrm{C}_{0}\left(\overline{\mathrm{U}_{\mathrm{j}}}\right), A\right)_{\mathrm{g}}=0 .
$$

Let $X \subset M$ be any G-invariant, compact subset. The proof of Proposition 3.5 is based on the following fact.

Lemma 3.6. If $A$ is separable, then

$$
\mathrm{KK}_{\mathrm{G}}\left(\mathrm{A}, \mathrm{C}_{0}(\mathrm{X})\right)_{\mathrm{g}}=0
$$

If $\mathrm{A}$ is $\sigma$-unital, then

$$
\mathrm{KK}_{\mathrm{G}}\left(\mathrm{C}_{0}(\mathrm{X}), A\right)_{\mathrm{g}}=0 .
$$

Proof. We will use an induction argument based on exact sequences in KKtheory. We work out the details for (3.4). Then (3.5) can be proved in the same way, with exact sequences in the second entry in KK-theory replaced by the corresponding exact sequences in the first entry. The conditions that $A$ is separable or $\sigma$-unital imply that these exact sequences exist.

For $j, n \in \mathbb{N}$, write $X_{j}:={\overline{u_{j}}}_{X}$, and $Y_{n}:=X_{1} \cup \cdots \cup X_{n}$. Fix $n \in \mathbb{N}$, and consider the exact sequence of $\mathrm{C}^{*}$-algebras

$$
0 \rightarrow C_{0}\left(X_{n+1} \backslash Y_{n}\right) \rightarrow C_{0}\left(X_{n+1}\right) \rightarrow C_{0}\left(X_{n+1} \cap Y_{n}\right) \rightarrow 0 .
$$

It induces the exact triangle

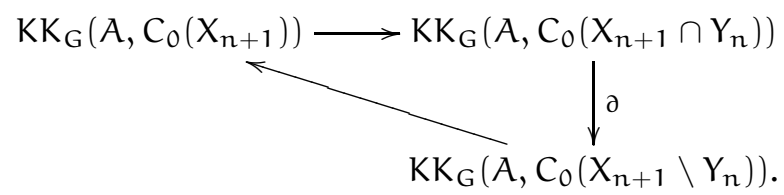


(See e.g. [11, Theorem 19.5.7].) By Proposition 3.4, we have

$$
K_{G}\left(A, C_{0}\left(X_{n+1}\right)\right)_{g}=K_{G}\left(A, C_{0}\left(X_{n+1} \cap Y_{n}\right)\right)_{g}=0 .
$$

Since localisation at $\mathrm{g}$ preserves exactness, we find that

$$
\mathrm{KK}_{\mathrm{G}}\left(A, \mathrm{C}_{0}\left(X_{n+1} \backslash Y_{n}\right)\right)_{\mathrm{g}}=0 .
$$

Using the exact sequence

$$
0 \rightarrow \mathrm{C}_{0}\left(\mathrm{Y}_{\mathrm{n}+1} \backslash \mathrm{Y}_{\mathrm{n}}\right) \rightarrow \mathrm{C}_{0}\left(\mathrm{Y}_{\mathrm{n}+1}\right) \rightarrow \mathrm{C}_{0}\left(\mathrm{Y}_{\mathrm{n}}\right) \rightarrow 0
$$

in a similar way, we obtain the exact triangle

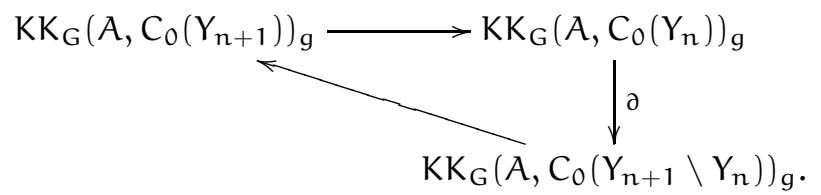

Since $Y_{n+1} \backslash Y_{n}=X_{n+1} \backslash Y_{n}$, the vanishing of (3.6) implies that

$$
\mathrm{KK}_{\mathrm{G}}\left(\mathrm{A}, \mathrm{C}_{0}\left(\mathrm{Y}_{\mathrm{n}+1}\right)\right)_{\mathrm{g}}=\mathrm{KK}_{\mathrm{G}}\left(\mathrm{A}, \mathrm{C}_{0}\left(\mathrm{Y}_{\mathrm{n}}\right)\right)_{\mathrm{g}} \text {. }
$$

Because $Y_{1}=X_{1}$, Proposition 3.4 implies that

$$
\mathrm{KK}_{\mathrm{G}}\left(A, \mathrm{C}_{0}\left(\mathrm{Y}_{1}\right)\right)_{\mathrm{g}}=0 .
$$

Since $X$ is compact, it can be covered by finitely many of the sets $X_{j}$. Hence the claim follows by induction on $n$.

Proof of Proposition 3.5 Let $\mathrm{U} \subset \mathrm{M}$ be a G-invariant, relatively compact open subset. Consider the exact sequence

$$
0 \rightarrow \mathrm{C}_{0}(\mathrm{U}) \rightarrow \mathrm{C}_{0}(\overline{\mathrm{U}}) \rightarrow \mathrm{C}_{0}(\mathrm{\partial u}) \rightarrow 0 .
$$

If $A$ is $\sigma$-unital, this induces the localised exact triangle

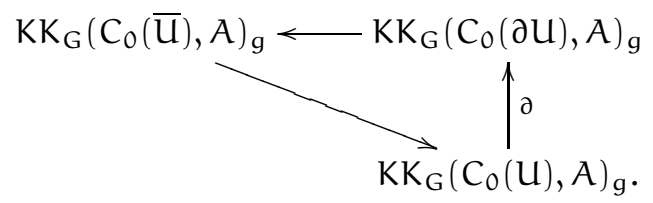

Lemma 3.6 implies that

$$
\mathrm{KK}_{\mathrm{G}}\left(\mathrm{C}_{0}(\overline{\mathrm{U}}), A\right)_{\mathrm{g}}=\mathrm{KK}_{\mathrm{G}}\left(\mathrm{C}_{0}(\partial \mathrm{U}), A\right)_{\mathrm{g}}=0 .
$$

So we find that $\mathrm{KK}_{\mathrm{G}}\left(\mathrm{C}_{0}(\mathrm{U}), A\right)_{\mathrm{g}}=0$.

Similarly, If $A$ is separable, we have the exact triangle

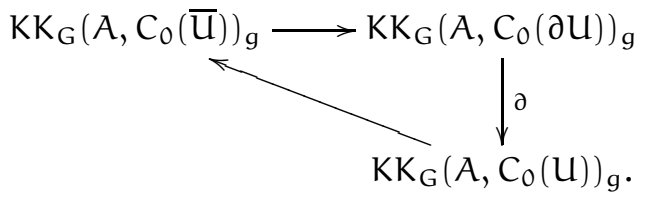


Applying Lemma 3.6 in the same way, we find that $\mathrm{KK}_{\mathrm{G}}\left(\mathrm{A}, \mathrm{C}_{0}(\mathrm{U})\right)_{\mathrm{g}}=0$. The equality (3.2) follows, because $M$ is the direct limit of sets $U$ as above, and because KK-theory commutes with direct limits in the second entry.

Remark 3.7. The reason why (3.3) does not hold if $U$ is replaced by $M$, and hence why Theorem 2.3 has to be stated more subtly than Theorem 2.1, is that KK-theory does not commute with direct limits in the first entry. For example, the domain of the analytic assembly map in the Baum-Connes conjecture [7] is the representable $\mathrm{K}$-homology group

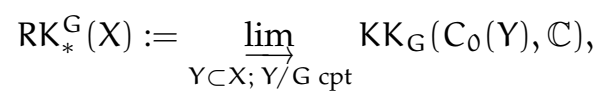

for a locally compact Hausdorff space $X$ on which a locally compact group $G$ acts properly. This does not equal the usual $\mathrm{K}$-homology group $\mathrm{KK}_{\mathrm{G}}\left(\mathrm{C}_{0}(\mathrm{X}), \mathbb{C}\right)$ in general.

\subsection{Proofs of localisation results}

Proof of Theorem 2.1 Consider the exact sequence

$$
0 \rightarrow C_{0}\left(M \backslash M^{g}\right) \rightarrow C_{0}(M) \stackrel{\left(j_{M g}^{M}\right)^{*}}{\longrightarrow} C_{0}\left(M^{g}\right) \rightarrow 0 .
$$

It induces the exact triangle

$$
\begin{aligned}
& \mathrm{KK}_{\mathrm{G}}\left(\mathrm{A}, \mathrm{C}_{\mathrm{O}}(\mathrm{M})\right) \stackrel{\left(\mathrm{j}_{\mathrm{M}^{\mathrm{g}}}^{\mathrm{M}}\right)^{*}}{\longrightarrow} \mathrm{KK} \mathrm{K}_{\mathrm{G}}\left(\mathrm{A}, \mathrm{C}_{\mathrm{O}}\left(\mathrm{M}^{\mathrm{g}}\right)\right) \\
& \downarrow^{2} \\
& \mathrm{KK}_{\mathrm{G}}\left(A, \mathrm{C}_{0}\left(M \backslash M^{\mathrm{g}}\right)\right) \text {. }
\end{aligned}
$$

After localisation at $\mathrm{g}$, the first part of Proposition 3.5 yields the exact triangle

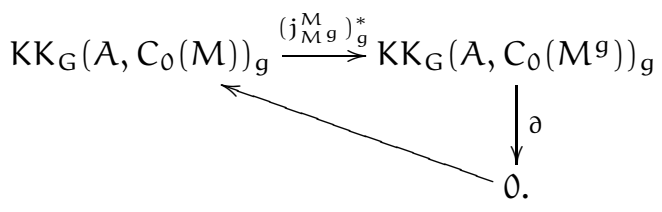

Proof of Theorem 2.3 Let $\mathrm{U}$ and $\mathrm{V}$ be as in Theorem 2.3. Similarly to the proof of Theorem 2.1, we have an exact triangle

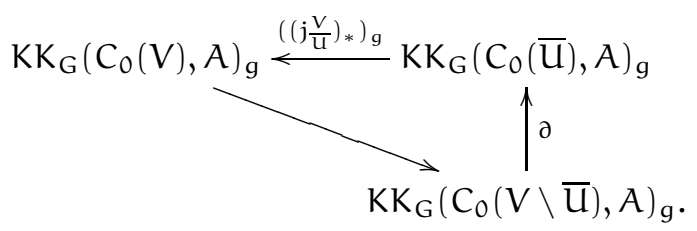

Because $V \backslash \bar{U}$ is a relatively compact subset of $M \backslash M^{g}$, the second part of Proposition 3.5 implies that the bottom localised KK-group in this triangle equals zero. 


\section{Kasparov's index theorem}

In the proof of the Atiyah-Segal-Singer fixed point theorem, the Atiyah-Singer index theorem is used to relate topological and analytical indices to each other. In the noncompact case discussed in this paper, a roughly similar role is played by an index theorem of Kasparov. We state Kasparov's index theorem in Subsection 4.1. In Subsection 4.2, we discuss the fibrewise Bott element for the normal bundle of a submanifold in KK-theory, which is dual to the class of the Dolbeault-Dirac operator in a sense. This Bott element will play an important role in the proof of Theorem 2.9 In Subsection 4.4. we show how the Bott element can be used to deduce the Atiyah-Singer index theorem from Kasparov's index theorem in the compact case. (The main step in the argument used there will be used in the proof of Theorem 2.9.)

Most of the material in this section is based on [6, 26], and explanations to the authors by Kasparov. Although the results here are not ours, we found it worthwhile to include the details, because they have not appeared in print yet.

\subsection{The index theorem}

To state the theorem, we recall the definition of the Dolbeault operator class

$$
\left[\mathrm{D}_{\mathrm{TM}}\right] \in \mathrm{KK}_{\mathrm{G}}(\mathrm{TM}, \mathbb{C})
$$

in [26. Definition 2.8]. The tangent bundle T(TM) of TM has a natural almost complex structure J. For $m \in M$ and $v \in \mathrm{T}_{\mathrm{m}} M$, we have

$$
\mathrm{T}_{v}(\mathrm{TM})=\mathrm{T}_{\mathrm{m}} M \oplus \mathrm{T}_{v}\left(\mathrm{~T}_{\mathrm{m}} M\right)=\mathrm{T}_{\mathrm{m}} M \oplus \mathrm{T}_{\mathrm{m}} M .
$$

With respect to this decomposition, the almost complex structure $J$ is given by the matrix $\left[\begin{array}{cc}0 & 1 \\ -1 & 0\end{array}\right]$. Let $\bar{\partial}+\bar{\partial}^{*}$ be the Dolbeault-Dirac operator on smooth sections of the vector bundle $\bigwedge^{0, *} \mathrm{~T}^{*}(\mathrm{TM}) \rightarrow \mathrm{TM}$, for this almost complex structure. We will identify this vector bundle with $\tau_{M}^{*} \wedge \mathrm{TM}_{\mathbb{C}} \rightarrow \mathrm{TM}$. The class (4.1) is the class of this operator, as in (2.1). In our arguments however, it will be more convenient to use the $\operatorname{Spin}^{\mathrm{c}}$-Dirac operator $\mathrm{D}_{\mathrm{TM}}$, on the same vector bundle. This defines the same K-homology class as $\bar{\partial}+\bar{\partial}^{*}$.

Definition 4.1. The topological index is the map

$$
\text { index }_{\mathrm{t}}: \mathrm{KK}_{\mathrm{G}}(\mathrm{M}, \mathrm{TM}) \rightarrow \mathrm{KK}_{\mathrm{G}}(\mathrm{M}, \mathrm{pt})
$$

given by the Kasparov product with $\left[\mathrm{D}_{\mathrm{TM}}\right]$.

Consider the principal symbol $\tilde{\sigma}_{\mathrm{D}}:=\frac{\sigma_{\mathrm{D}}}{\sqrt{\sigma_{\mathrm{D}}^{2}+1}}$ of the operator $\frac{\mathrm{D}}{\sqrt{\mathrm{D}^{2}+1}}$. For $\mathrm{f} \in \mathrm{C}_{0}(M)$, we have for all $\mathrm{m} \in M$ and $v \in \mathrm{T}_{\mathrm{m}} M$,

$$
f(m)\left(1-\tilde{\sigma}_{D}(v)^{2}\right)=f(m)\left(\sigma_{D}(v)^{2}+1\right)^{-1} .
$$

Since the operator D is elliptic and of positive order, this expression tends to zero as $m$ or $v$ tends to infinity. It therefore defines a compact operator on 
the Hilbert $C_{0}(T M)$-module $\Gamma_{0}\left(\tau_{M}^{*} E\right)$, analogously to the proof of Lemma 3.2 Therefore, the triple

$$
\left(\Gamma_{0}\left(\tau_{M}^{*} E\right), \tilde{\sigma}_{D}, \pi_{T M} \circ \tau_{M}^{*}\right)
$$

is a G-equivariant Kasparov $\left(C_{0}(M), C_{0}(T M)\right)$-cycle. Here $\pi_{T M}: C_{b}(T M) \rightarrow$ $\mathcal{B}\left(\Gamma_{0}\left(\tau_{M}^{*} E\right)\right)$ is given by pointwise multiplication. Denote by

$$
\left[\sigma_{\mathrm{D}}\right] \in \mathrm{KK}_{\mathrm{G}}(\mathrm{M}, \mathrm{TM}) .
$$

the class of (4.2). In view of the following lemma, this symbol class is a natural generalisation of the K-theory symbol class defined in [5] when $M$ is compact.

Lemma 4.2. If $M$ is compact, consider the map $\mathrm{p}^{M}$ from $M$ to a point. The image

$$
\mathrm{p}_{*}^{\mathrm{M}}\left[\sigma_{\mathrm{D}}\right] \in \mathrm{K}_{\mathrm{G}}^{*}(\mathrm{TM})
$$

is the usual symbol class.

Proof. Since $\pi_{\mathrm{TM}} \circ \tau_{M}^{*} \circ\left(\mathrm{p}^{M}\right)^{*}$ is the representation of $\mathbb{C}$ in $\Gamma_{0}\left(\tau_{M}^{*} E\right)$ by scalar multiplication, we have

$$
p_{*}^{M}\left[\sigma_{D}\right]=\left[\Gamma_{0}\left(\tau_{M}^{*} E\right), \tilde{\sigma}_{D}\right] \quad \in K K_{G}(p t, T M) .
$$

This is corresponds to the class

$$
\left[\sigma_{D^{+}}: \tau_{M}^{*} E^{+} \rightarrow \tau_{M}^{*} E^{-}\right] \in K_{G}^{0}(T M)
$$

in the sense of [29, Ch. III eq. (1.7)], where TM is identified with the open unit ball bundle $\mathrm{BM}$ over $M$. (Restricting $\sigma_{\mathrm{D}}+$ to $\mathrm{BM}$ and then identifying $\mathrm{BM} \cong$ TM has the same effect as normalising $\sigma_{\mathrm{D}^{+}}$.) The lemma is then proved.

We conclude this subsection by stating Kasparov's index theorem, which will be used to obtain a cohomological formula for the $g$-index.

Theorem 4.3 (Kasparov's index theorem [26, Theorem 4.2]). The K-homology class of the elliptic operator in (2.1) is equal to the topological index of its symbol class (4.3), i.e.,

$$
[\mathrm{D}]=\operatorname{index}_{\mathrm{t}}\left[\sigma_{\mathrm{D}}\right] \in \mathrm{KK}_{\mathrm{G}}(\mathrm{M}, \mathbb{C}) \text {. }
$$

Remark 4.4. In [26, Theorem 4.2], the operator in question is assumed to be properly supported, which is not true for the operator $\frac{D}{\sqrt{1+D^{2}}}$ in general. However, let $\left\{\chi_{j}\right\}_{j=1}^{\infty}$ be sequence of G-invariant, compactly supported functions, such that $\left\{\chi_{j}^{2}\right\}_{j=1}^{\infty}$ is a partition of unity. (This exists since $G$ is compact.) Then the operator

$$
\sum_{j=1}^{\infty} x_{j} \frac{D}{\sqrt{1+D^{2}}} x_{j}
$$

is properly supported, and also satisfies the other assumptions of [26, Theorem 4.2]. Since this operator defines the same K-homology class as $\frac{D}{\sqrt{1+D^{2}}}$, we can apply [26, Theorem 4.2] to the class of the latter operator in this way. 


\subsection{The Bott element}

If $S$ is a closed (as a topological subspace, i.e. not necessarily compact), Ginvariant submanifold of $M$, then the Dolbeault operator classes on TS and on a tubular neighbourhood of TS in TM are related by a (fibrewise) Bott element. This is a technical tool that will be used several times in the paper. The material here is analogous to Definition 2.6 and Theorem 2.7 in [26].

Consider the tangent bundle projections

$$
\begin{gathered}
\tau_{S}: \mathrm{TS} \rightarrow S ; \\
\tau_{\mathrm{N}}: \mathrm{TN} \rightarrow \mathrm{N} .
\end{gathered}
$$

Denote by $\pi: N \rightarrow S$ the normal bundle of $S$ in $M$. Let T $\pi: T N \rightarrow$ TS be the tangent map of $\pi$. It again defines a vector bundle. The following diagram commutes:

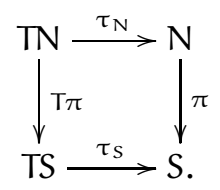

This defines a vector bundle $\mathrm{TN} \rightarrow \mathrm{S}$. Consider the vector bundle

$$
\bigwedge \widetilde{\mathrm{N}}_{\mathbb{C}}:=\mathrm{T} \pi^{*}\left(\tau_{\mathrm{S}}^{*} \bigwedge \mathrm{N} \otimes \mathbb{C}\right) \rightarrow \mathrm{TN}
$$

Let $s \in \mathrm{S}$. Then

$$
\begin{aligned}
(\mathrm{TN})_{s} & :=\mathrm{T}^{-1}\left(\tau_{\mathrm{S}}^{-1}(\mathrm{~s})\right) \\
& =\tau_{\mathrm{N}}^{-1}\left(\mathrm{~N}_{\mathrm{s}}\right) \\
& =\mathrm{T}_{\mathrm{s}} \mathrm{S} \times \mathrm{N}_{\mathrm{s}} \times \mathrm{N}_{\mathrm{s}} .
\end{aligned}
$$

Let $w \in(\mathrm{TN})_{s}$, and let $(\eta, \zeta) \in \mathrm{N}_{\mathrm{s}} \times \mathrm{N}_{\mathrm{s}}$ be the projection of $w$ according to this decomposition. Note that

$$
\left(\bigwedge \widetilde{N}_{\mathbb{C}}\right)_{w}=\bigwedge N_{s} \otimes \mathbb{C}
$$

We define the vector bundle endomorphism $B$ of $\wedge \widetilde{N}_{\mathbb{C}}$ by

$$
\mathrm{B}_{w}=\operatorname{ext}(\zeta+\sqrt{-1} \eta)-\operatorname{int}(\zeta+\sqrt{-1} \eta)
$$

for all $s, w, \eta$ and $\zeta$ as above. Here ext denotes the wedge product, and int denotes contraction. With respect to the grading of $\wedge \widetilde{N}_{\mathbb{C}}$ by even and odd exterior powers, the operator $B$ is odd.

As $B$ is fibrewise selfadjoint, we have the bounded operator $B\left(1+B^{2}\right)^{-\frac{1}{2}}$ on $\Gamma_{0}\left(\mathbb{T N}, \wedge \widetilde{N}_{\mathbb{C}}\right)$. The space $\Gamma_{0}\left(\mathbb{T N}, \wedge \widetilde{N}_{\mathbb{C}}\right)$ is a right Hilbert $C_{0}(T N)$-module in the usual way, with respect to pointwise multiplication by functions and the pointwise inner product. Consider the representation

$$
\tilde{\pi}_{\mathrm{TS}}:=\pi_{\mathrm{TN}} \circ \mathrm{T} \pi^{*}: \mathrm{C}_{0}(\mathrm{TS}) \rightarrow \mathcal{B}\left(\Gamma_{0}\left(\mathrm{TN}, \wedge \widetilde{\mathrm{N}}_{\mathbb{C}}\right)\right),
$$

where $\pi_{\mathrm{TN}}$ is given by pointwise multiplication by functions in $C_{b}(T N)$. 
Lemma 4.5. The triple

$$
\left(\Gamma_{0}\left(\mathrm{TN}, \wedge \widetilde{N}_{\mathbb{C}}\right), \mathrm{B}\left(1+\mathrm{B}^{2}\right)^{-\frac{1}{2}}, \tilde{\pi}_{\mathrm{TS}}\right)
$$

is a $\mathrm{G}$-equivariant Kasparov ( $\left.\mathrm{C}_{0}(\mathrm{TS}), \mathrm{C}_{0}(\mathrm{TN})\right)$-cycle.

Proof. Let $\mathrm{f} \in \mathrm{C}_{0}$ (TS). Since $\mathrm{B}\left(1+\mathrm{B}^{2}\right)^{-\frac{1}{2}}$ is a vector bundle endomorphism, it commutes with $\tilde{\pi}_{\mathrm{TS}}(\mathrm{f})$. Moreover, we have for all $w \in(\mathrm{TN})_{\mathrm{s}}$ as above,

$$
\left(\tilde{\pi}_{\mathrm{TS}}(\mathrm{f})\left(1-\left[\mathrm{B}\left(1+\mathrm{B}^{2}\right)^{-\frac{1}{2}}\right]^{2}\right)\right)_{w}=\frac{\mathrm{f}(v)}{1+\|\eta\|^{2}+\|\zeta\|^{2}}
$$

with $v:=\mathrm{T} \pi(w) \in \mathrm{T}_{\mathrm{s}} S$. This defines a function in $\mathrm{C}_{0}(\mathrm{TN})$, and hence acts on the Hilbert $C_{0}(T N)$-module $\Gamma_{0}\left(\mathbb{T N}, \wedge \widetilde{N}_{\mathbb{C}}\right)$ as a compact operator. As $g$ preserves the metric $T N$, the operator $B\left(1+B^{2}\right)^{-\frac{1}{2}}$ is G-equivariant.

Definition 4.6. The (fibrewise) Bott element of the normal bundle $N \rightarrow S$ is the class

$$
\beta_{N} \in K K_{G}(T S, T N)
$$

of the cycle (4.6).

\subsection{The Bott element and Dolbeault classes}

The Bott element is useful to us because of the following property. This was used in [26, bottom paragraph on p. 30]; we work out some details of the proof in this subsection.

Proposition 4.7. Under the Kasparov product $\mathrm{KK}_{\mathrm{G}}(\mathrm{TS}, \mathrm{TN}) \times \mathrm{KK}_{\mathrm{G}}(\mathrm{TN}, \mathrm{pt}) \rightarrow$ $\mathrm{KK}_{\mathrm{G}}(\mathrm{TS}, \mathrm{pt})$, one has

$$
\beta_{\mathrm{N}} \otimes_{\mathrm{TN}}\left[\mathrm{D}_{\mathrm{TN}}\right]=\left[\mathrm{D}_{\mathrm{TS}}\right] .
$$

To prove this proposition, one can use the part $D_{1}$ of the $\operatorname{Spin}^{\mathrm{c}}$-Dirac operator $D_{T N}$ acting in the fibre directions of $\mathrm{TN} \rightarrow T S$. For $s \in S$ and $v \in T_{S} S$, we have $T \pi^{-1}(v)=N_{s} \oplus T_{v} N$. Let a be the rank of $N$, and let $\left\{f_{1}, \ldots, f_{a}\right\}$ be a local orthonormal frame of $\mathrm{N} \rightarrow \mathrm{S}$. This defines coordinate functions $\kappa_{j}$ and $\lambda_{j}$ on the parts $N_{s}$ and $T_{v} N$ of the fibres $T \pi^{-1}(v)$ of $T N \rightarrow T S$, respectively. For $j=1, \ldots, a$, consider the vector bundle endomorphisms

$$
e_{j}:=\operatorname{ext}\left(f_{j}\right)-\operatorname{int}\left(f_{j}\right) \quad \text { and } \quad \epsilon_{j}:=\operatorname{ext}\left(f_{j}\right)+\operatorname{int}\left(f_{j}\right)
$$

of $\bigwedge N \otimes \mathbb{C} \rightarrow S$, pulled back along (4.5) to endomorphisms of $\bigwedge \widetilde{N}_{\mathbb{C}} \rightarrow T N$. Then $D_{1}$ is the operator

$$
D_{1}:=\sum_{j=1}^{a} e_{j} \frac{\partial}{\partial \kappa_{j}}+\sqrt{-1} \epsilon_{j} \frac{\partial}{\partial \lambda_{j}}
$$

on $\Gamma^{\infty}\left(\mathrm{TN}, \wedge \widetilde{\mathrm{N}}_{\mathbb{C}}\right)$. This can be viewed as a family of operators on the fibres of $\mathrm{TN} \rightarrow \mathrm{TS}$. 
It defines a class in KK-theory as follows. Let $\Gamma_{\mathcal{c}}\left(T N, \wedge \widetilde{N}_{\mathbb{C}}\right)$ be the space of continuous compactly supported sections of $\Lambda \widetilde{N}_{\mathbb{C}}$. Let $\varepsilon_{0}$ be the completion of this space into a Hilbert $\mathrm{C}_{0}(\mathrm{TS})$-module with respect to the following $\mathrm{C}_{0}$ (TS)valued inner product:

$$
\langle f, h\rangle(v):=\int_{T^{-1}(v)} \overline{f(t)} h(t) d t
$$

for $\mathrm{f}, \mathrm{h} \in \Gamma_{\mathrm{c}}\left(\mathrm{TN}, \wedge \widetilde{\mathrm{N}}_{\mathbb{C}}\right)$ and $v \in \mathrm{TS}$. The operator $\mathrm{D}_{1}$ gives rise to the class

$$
\left[\mathrm{D}_{1}\right]:=\left[\mathcal{E}_{0}, \mathrm{D}_{1}\left(1+\mathrm{D}_{1}^{2}\right)^{-\frac{1}{2}}, \pi_{\mathrm{TN}}\right] \in \mathrm{KK}_{\mathrm{G}}(\mathrm{TN}, \mathrm{TS}) .
$$

Lemma 4.8. We have

$$
\left[\mathrm{D}_{1}\right] \otimes \mathrm{TS}_{\mathrm{TS}}\left[\mathrm{D}_{\mathrm{TS}}\right]=\left[\mathrm{D}_{\mathrm{TN}}\right] \quad \in \mathrm{KK}_{\mathrm{G}}(\mathrm{TN}, \mathrm{pt}) .
$$

Proof. Regarding $\mathrm{N}$ as an open subset of $\mathrm{M}$, we identify their tangent bundles when restricted to $S$, i.e., $\left.T N\right|_{S}=\left.T M\right|_{S}$. Therefore, as vector bundles over $\mathrm{TN}$, we have

$$
\begin{aligned}
\Lambda \widetilde{N}_{\mathbb{C}} \otimes T \pi^{*} \tau_{\mathrm{S}}^{*} \bigwedge T S_{\mathbb{C}} & =T \pi^{*} \tau_{\mathrm{S}}^{*} \bigwedge \mathrm{N}_{\mathbb{C}} \otimes T \pi^{*} \tau_{\mathrm{S}}^{*} \wedge \mathrm{TS} S_{\mathbb{C}} \\
& =\mathrm{T} \pi^{*} \tau_{\mathrm{S}}^{*} \bigwedge(\mathrm{N} \oplus \mathrm{TS})_{\mathbb{C}}=\mathrm{T} \pi^{*} \tau_{\mathrm{S}}^{*} \bigwedge\left(\left.\mathrm{TM}\right|_{\mathrm{S}}\right)_{\mathbb{C}} \\
& =\mathrm{T} \pi^{*} \tau_{\mathrm{S}}^{*} \bigwedge\left(\left.\mathrm{TN}\right|_{\mathrm{S}}\right)_{\mathbb{C}}=\tau_{\mathrm{N}}^{*} \wedge \mathrm{T} \mathrm{N}_{\mathbb{C}} .
\end{aligned}
$$

The last equality follows from commutativity of (4.5). Thus, as Hilbert spaces with representations of $C_{0}(T N)$,

$$
\varepsilon_{0} \otimes \mathrm{C}_{0}(\mathrm{TS}) \mathrm{L}^{2}\left(\mathrm{TS}, \tau_{\mathrm{S}}^{*} \wedge \mathrm{TS}_{\mathbb{C}}\right) \cong \mathrm{L}^{2}\left(\mathrm{TN}, \tau_{\mathrm{N}}^{*} \wedge \mathrm{TN}_{\mathbb{C}}\right) .
$$

Under this identification, we have

$$
\mathrm{D}_{1} \otimes 1+1 \otimes \mathrm{D}_{\mathrm{TS}}=\mathrm{D}_{\mathrm{TN}} .
$$

(Here we use graded tensor products.) Consider the bounded operator

$$
\mathrm{F}:=\frac{\mathrm{D}_{1} \otimes 1+1 \otimes \mathrm{D}_{\mathrm{TS}}}{\sqrt{1+\mathrm{D}_{1}^{2} \otimes 1+1 \otimes \mathrm{D}_{\mathrm{TS}}^{2}}}
$$

on $\mathcal{E}_{0} \otimes \mathrm{C}_{0}(\mathrm{TS}) \mathrm{L}^{2}\left(\mathrm{TS}, \tau_{\mathrm{S}}^{*} \wedge \mathrm{TS}_{\mathbb{C}}\right)$. Write

$$
\mathrm{F}_{1}:=\frac{\mathrm{D}_{1}}{\sqrt{1+\mathrm{D}_{1}^{2}}} ; \quad \mathrm{F}_{\mathrm{TS}}:=\frac{\mathrm{D}_{\mathrm{TS}}}{\sqrt{1+\mathrm{D}_{\mathrm{TS}}^{2}}} .
$$

We can verify that $\mathrm{F}$ is an $1 \otimes \mathrm{F}_{\mathrm{TS}}$-connection, and the graded commutator $\left[\mathrm{F}_{1} \otimes 1, \mathrm{~F}\right]$ is positive modulo compact operators. Therefore, by [11, Definition 18.4.1] the Kasparov product $\left[\mathrm{D}_{1}\right] \otimes_{\mathrm{TS}}\left[\mathrm{D}_{\mathrm{TS}}\right]$ is represented by the operator $\mathrm{F}$ on $\mathrm{L}^{2}\left(\mathrm{TN}, \tau_{\mathrm{N}}^{*} \wedge \mathrm{TN}_{\mathbb{C}}\right)$. The lemma is then proved. 
Lemma 4.9. The product

$$
\beta_{\mathrm{N}} \otimes_{\mathrm{TN}}\left[\mathrm{D}_{1}\right] \quad \in \mathrm{KK}_{\mathrm{G}}(\mathrm{TS}, \mathrm{TS}) .
$$

is the ring identity.

Proof. The idea is that in this product, we pair fibrewise Bott classes and Dolbeault classes, and thus obtain the trivial line bundle over TS. To see this, observe first the isomorphism

$$
\Gamma_{\mathrm{c}}\left(\mathrm{TN}, \wedge \widetilde{N}_{\mathbb{C}}\right) \otimes_{\mathrm{C}_{\mathrm{c}}(\mathrm{TN})} \Gamma_{\mathrm{c}}\left(\mathrm{TN}, \wedge \widetilde{N}_{\mathbb{C}}\right) \cong \Gamma_{\mathrm{c}}\left(\mathrm{TN}, \wedge \widetilde{N}_{\mathbb{C}} \otimes \wedge \widetilde{N}_{\mathbb{C}}\right)
$$

as $\mathrm{C}_{\mathrm{c}}(\mathrm{TS})$-modules. Denote by $\mathcal{E}^{\prime}$ the completion of the right-hand side under the $\mathrm{C}_{0}$ (TS)-valued inner product defined in a similar way as (4.7). It can be checked that

$$
F_{0}:=\frac{B \otimes 1+1 \otimes D_{1}}{\sqrt{1+B^{2} \otimes 1+1 \otimes D_{1}^{2}}}
$$

is a $1 \otimes \frac{D_{1}}{\sqrt{1+D_{1}^{2}}}$-connection, and that for all a in $C_{0}(T S)$, the operator

$$
\tilde{\pi}_{\mathrm{TS}}(\mathrm{a})\left[\mathrm{B}\left(1+\mathrm{B}^{2}\right)^{-\frac{1}{2}} \otimes 1, \mathrm{~F}_{0}\right] \tilde{\pi}_{\mathrm{TS}}(\mathrm{a})^{*}
$$

is positive modulo compact operators on $\mathcal{E}^{\prime}$. Hence, the Kasparov product of $\beta_{N}$, given by (4.6), and the class $\left[D_{1}\right]$, given by (4.8), is equal to

$$
\left[\mathcal{E}^{\prime}, \mathrm{F}_{0}, \tilde{\pi}_{\mathrm{TS}}\right] \in \mathrm{KK}_{\mathrm{G}}(\mathrm{TS}, \mathrm{TS}) \text {. }
$$

As in the proof of [26. Theorem 2.7 (2)], we apply the rotation homotopy

$$
F_{t}:=\frac{\left(B+\sin (t) D_{1}\right) \otimes 1+1 \otimes \cos (t) D_{1}}{\sqrt{1+\left(B^{2}+\sin (t)^{2} D_{1}^{2}\right) \otimes 1+1 \otimes \cos (t)^{2} D_{1}^{2}}},
$$

for $t \in[0, \pi / 2]$. Then the operator $F_{0}$ in the cycle (4.13) is transformed into $\mathrm{F}_{\pi / 2}=\mathrm{F}^{\prime} \otimes 1$, where

$$
F^{\prime}:=\left(B+D_{1}\right)\left(1+B^{2}+D_{1}^{2}\right)^{-\frac{1}{2}} .
$$

In summary, $\beta_{N}$ and $\left[D_{1}\right]$ are families of operators indexed by TS whose Kasparov product is represented by $F^{\prime}$. At every $v \in T_{s} S$, the square of $B+D_{1}$ is the harmonic oscillator operator

$$
\sum_{j=1}^{a}\left(\kappa_{j}^{2}+\lambda_{j}^{2}-\frac{\partial}{\partial \kappa_{j}^{2}}-\frac{\partial^{2}}{\partial \lambda_{j}^{2}}\right)+2 \operatorname{deg}-a
$$

on $T^{-1}(v) \cong N_{s} \times N_{s}$. (Here deg is the degree in $\bigwedge N$.) It has a one dimensional kernel, concentrated in degree zero, generated by

$$
(\eta, \zeta) \mapsto e^{-\frac{\|\eta\|^{2}+\|\zeta\|^{2}}{2}} \in \mathrm{C}_{0}\left(\mathrm{~N}_{s} \times \mathrm{N}_{s}\right) .
$$

Thus, over each fibre, $F^{\prime}$ is a Fredholm operator with index 1 , and $\beta_{N} \otimes_{T N}\left[D_{1}\right]$ is equal to the exterior product of this Fredholm operator in $\mathrm{KK}_{\mathrm{G}}(\mathbb{C}, \mathbb{C})$ and $\left[\mathrm{C}_{0}\right.$ (TS), $\left.0, \pi_{\mathrm{TS}}\right] \in \mathrm{KK}_{\mathrm{G}}$ (TS, TS) both representing the respective ring identities. Hence the claim follows. 
Proof of Proposition 4.7 Using Lemmas 4.8 and 4.9, and associativity of the Kasparov product, we find that

$$
\beta_{\mathrm{N}} \otimes_{\mathrm{TN}}\left[\mathrm{D}_{\mathrm{TN}}\right]=\left(\beta_{\mathrm{N}} \otimes_{\mathrm{TN}}\left[\mathrm{D}_{1}\right]\right) \otimes_{\mathrm{TS}}\left[\mathrm{D}_{\mathrm{TS}}\right]=\left[\mathrm{D}_{\mathrm{TS}}\right] .
$$

This finishes the proof.

We will later need the restriction of the Bott element to TS. Consider the class

$$
\left[\tau_{S}^{*} \wedge N_{\mathbb{C}}\right]:=\left[\bigoplus_{j} \wedge^{2 j} \tau_{S}^{*} N \otimes \mathbb{C}\right]-\left[\bigoplus_{j} \wedge^{2 j+1} \tau_{S}^{*} N \otimes \mathbb{C}\right] \in K_{G}(T S, T S),
$$

defined as in Lemma3.2.

Lemma 4.10. We have

$$
\left(j_{\mathrm{TS}}^{\mathrm{TN}}\right)^{*} \beta_{\mathrm{N}}=\left[\tau_{\mathrm{S}}^{*} \wedge \mathrm{N}_{\mathbb{C}}\right] \quad \in \mathrm{KK}_{\mathrm{G}}(\mathrm{TS}, \mathrm{TS}) .
$$

Proof. The Hilbert $C_{0}(T S)$-module in $\left(j_{T S}^{T N}\right)^{*} \beta_{N}$ is $\Gamma_{0}\left(T S, \tau_{S}^{*} \wedge N_{\mathbb{C}}\right)$. Because $\left.B\right|_{T S}$ is the zero operator, the claim follows.

\subsection{The Atiyah-Singer index theorem}

Suppose for now that $M$ is compact and $G$ is trivial. Then Kasparov's index theorem reduces to the Atiyah-Singer index theorem, see [26, Remark 4.5]. We provide the details of this implication here, because these will used in the proof of Theorem 2.9.

Consider the Atiyah-Singer topological index map

$$
\operatorname{index}_{t}^{\mathrm{AS}}: \mathrm{KK}(\mathrm{pt}, \mathrm{TM}) \rightarrow \mathbb{Z},
$$

which maps a class $\sigma \in \mathrm{KK}(\mathrm{pt}, \mathrm{TM})$ to

$$
\int_{\mathrm{TM}} \operatorname{ch}(\sigma) \operatorname{Todd}(\mathrm{TM} \otimes \mathbb{C}) .
$$

Note that we do not have the factor $(-1)^{\operatorname{dim} M}$ in (4.15) as in [6, Theorem 2.12], because we use a different almost complex structure on TM than in [6, p. 554], giving the opposite orientation.

Lemma 4.11. As a map

$$
\mathrm{KK}(\mathrm{pt}, \mathrm{TM}) \rightarrow \mathrm{KK}(\mathrm{pt}, \mathrm{pt}),
$$

right multiplication by $\left[\mathrm{D}_{\mathrm{TM}}\right]$ is the Atiyah-Singer topological index.

Because of Lemma 4.11, Theorem 4.3 implies the Atiyah-Singer index theorem. Indeed, since $M$ is compact, the map $\mathrm{p}^{M}: M \rightarrow$ pt is proper. By functoriality of the Kasparov product, Lemma 4.11implies that the following diagram commutes:

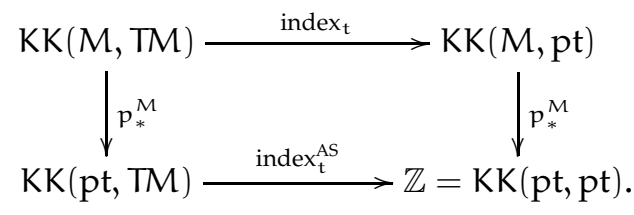


By Lemma 4.2, applying the map $p_{*}^{M}$ to both sides of (4.4), and using commutativity of the above diagram, one obtains the Atiyah-Singer index theorem.

Proof of Lemma 4.11 The proof is a reformulation of the arguments in [6], using KK-theory.

There are embeddings $M \hookrightarrow \mathbb{R}^{n}$ with normal bundle $N$ of rank a, and $\mathrm{TM} \hookrightarrow \mathrm{TR}^{n}=\mathbb{C}^{\mathrm{n}}$ with normal bundle $\mathrm{TN}$. As $\mathrm{N}$ is homeomorphic to a tubular neighbourhood of $M$ in $\mathbb{R}^{n}$, we can identify $\mathbb{T N}$ with an open neighbourhood of $T M$ in $\mathbb{C}^{n}$. (Note that here, the submanifold $S$ of $M$ in Subsection 4.2 is replaced by the submanifold $M$ of $\mathbb{R}^{n}$.)

Denote by

$$
\beta_{\mathrm{N}} \in \mathrm{KK}(\mathrm{TM}, \mathrm{TN})
$$

the fibrewise Bott element over TM in TN, in the sense of Definition 4.6 Then by Proposition 4.7

$$
\left[\mathrm{D}_{\mathrm{TM}}\right]=\beta_{\mathrm{N}} \otimes\left[\mathrm{D}_{\mathrm{TN}}\right] .
$$

The Chern character is compatible with the pairing of K-theory and K-homology. The Chern character of the Bott generator $\beta$ of $K^{0}\left(\mathbb{R}^{2}\right)$ is a generator of $\mathrm{H}^{2}\left(\mathbb{R}^{2}\right)$. As the Dolbeault class $\left[D_{\mathbb{R}^{2}}\right]$ of $\mathbb{R}^{2}$ is dual to $\beta$, its Chern character is the Poincaré dual of $\operatorname{ch}(\beta)$. So $\operatorname{ch}\left[D_{\mathbb{R}^{2}}\right]$ is the fundamental class $\left[\mathbb{R}^{2}\right]$ of $\mathbb{R}^{2}$. Similarly, working with the exterior Kasparov product of $n$ copies of $\beta$, we conclude that $\operatorname{ch}\left[D_{\mathbb{R}^{2 n}}\right]=\left[\mathbb{R}^{2 n}\right]$. Noting that $T \mathbb{R}^{n}=\mathbb{R}^{2 n}$, then by functoriality of the Chern character, we have

$$
\operatorname{ch}\left[D_{T N}\right]=\operatorname{ch}\left(\left(k_{T N}^{T \mathbb{R}^{n}}\right)_{*}\left[D_{T \mathbb{R}^{n}}\right]\right)=\left(k_{T N}^{T \mathbb{R}^{n}}\right)_{*} \operatorname{ch}\left[D_{T \mathbb{R}^{n}}\right]=\left(k_{T N}^{T \mathbb{R}^{n}}\right)_{*}\left[T \mathbb{R}^{n}\right]=[T N]
$$

Thus, the Chern character of $\left[D_{T N}\right]$ is the fundamental class $[T N] \in H_{2 n}(T N)$. Let $\sigma \in \mathrm{KK}(\mathrm{pt}, \mathrm{TM})$ be given. Then (4.16) and (4.17) imply that

$$
\sigma \otimes_{\mathrm{TM}}\left[\mathrm{D}_{\mathrm{TM}}\right]=\int_{\mathrm{TN}} \operatorname{ch}(\sigma) \wedge \operatorname{ch}\left(\beta_{\mathrm{N}}\right) .
$$

The Thom isomorphism $\psi_{\mathrm{TN}}: \mathrm{H}^{*}(\mathrm{TM}) \rightarrow \mathrm{H}^{*}(\mathrm{TN})$ (mapping between compactly supported cohomologies) is an isomorphism of $\mathrm{H}^{*}(\mathrm{TM})$-modules. So we can rewrite the integral (4.18) as

$$
\begin{aligned}
& \int_{\mathrm{TN}} \operatorname{ch}(\sigma) \wedge \operatorname{ch}\left(\beta_{\mathrm{N}}\right)=\int_{\mathrm{TM}} \psi_{\mathrm{TN}}^{-1}(\operatorname{ch}(\sigma)\left.\wedge \operatorname{ch}\left(\beta_{\mathrm{N}}\right)\right) \\
&=\int_{\mathrm{TM}} \operatorname{ch}(\sigma) \wedge \psi_{\mathrm{TN}}^{-1}\left(\operatorname{ch}\left(\beta_{\mathrm{N}}\right)\right) .
\end{aligned}
$$

To calculate $u:=\psi_{\mathrm{TN}}^{-1}\left(\operatorname{ch}\left(\beta_{\mathrm{TN}}\right)\right)$, we make use of the following diagram:

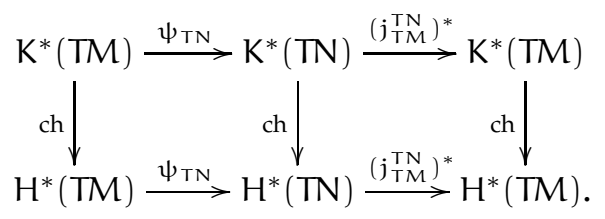


Note that in the second line, the composition is equal to the exterior product by the Euler class $e(T N)$. In the above diagram, we have by Lemma 4.10 ,

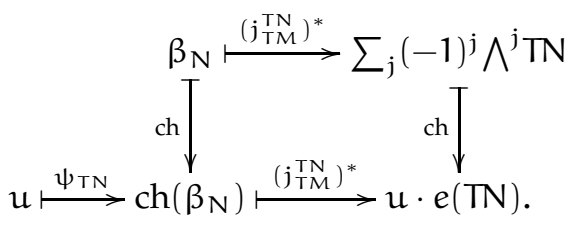

As the above square commutes by functoriality of the Chern character, and since $\mathbb{T N}=\tau_{M}^{*} N_{\mathbb{C}}$ and $N_{\mathbb{C}} \oplus(T M \otimes \mathbb{C})=M \times \mathbb{C}^{n}$, we obtain

$u=\frac{\operatorname{ch}\left(\sum_{j}(-1)^{j} \bigwedge^{j} T N\right)}{e(T N)}=\tau_{M}^{*}\left(\frac{e(T M)}{\left.\operatorname{ch}\left(\sum_{j}(-1)^{j} \bigwedge^{j} T M\right)\right)}\right)=\tau_{M}^{*}(\operatorname{Todd}(T M \otimes \mathbb{C}))$.

Therefore, together with (4.18) and (4.19) one has

$$
\sigma_{\mathrm{TM}} \otimes\left[\mathrm{D}_{\mathrm{TM}}\right]=\int_{\mathrm{TM}} \operatorname{ch}(\sigma) \operatorname{Todd}(\mathrm{TM} \otimes \mathbb{C}),
$$

and the lemma is proved.

\section{Proof of the fixed point formula}

After proving Theorems 2.1 and 2.3 and discussing Kasparov's index theorem, we are ready to prove Theorem 2.9

We start in Subsection 5.1, by generalising Gysin maps, or wrong-way functoriality maps in K-theory, that play a key role in [5]. We use these generalised Gysin maps in Subsection 5.2 to set up the commutative diagrams we need. We discuss a map defined by evaluating characters at $g$ in Subsection 5.3. Then we introduce a class in the topological K-theory of TM, localised at $\mathrm{g}$, defined by the principal symbol of $\mathrm{D}$. The properties of that class allow us to finish the proof of Theorem 2.9 .

\subsection{Gysin maps}

Let $S \subset M$ be a G-invariant submanifold, with inclusion map $j_{S}^{M}: S \hookrightarrow M$. (In the applications of what follows, $S$ will be a connected component of the fixed point set $M^{g}$.) Let $N \rightarrow S$ be the normal bundle of $S$ in $M$. The inclusion map $j_{\mathrm{TS}}^{\mathrm{TN}}: \mathrm{TS} \hookrightarrow \mathrm{TN}$ induces a map

$$
\left(\mathrm{j}_{\mathrm{TS}}^{\mathrm{TN}}\right)^{*}: \mathrm{C}_{0}(\mathrm{TN}) \rightarrow \mathrm{C}_{0}(\mathrm{TS})
$$

by restriction. We identify $\mathrm{TN}$ with an open neighbourhood of TS in TM, via a G-equivariant embedding $\mathrm{TN} \hookrightarrow \mathrm{TM}$. In this way, we have the injective map

$$
k_{T N}^{T M}: C_{0}(T N) \hookrightarrow C_{0}(T M),
$$

defined by extending functions by zero. 
Definition 5.1. Let $A$ be any $G-C^{*}$ algebra. The map $\left(j_{T S}^{T M}\right)_{!}: K_{G}\left(A, C_{0}(T S)\right) \rightarrow$ $\mathrm{KK}_{\mathrm{G}}\left(\mathrm{A}, \mathrm{C}_{\mathrm{O}}(\mathrm{TM})\right)$ is the composition

$$
\mathrm{KK}_{\mathrm{G}}\left(\mathrm{A}, \mathrm{C}_{0}(\mathrm{TS})\right) \stackrel{-\otimes \mathrm{C}_{0}(\mathrm{TS}) \beta_{\mathrm{N}}}{\longrightarrow} \mathrm{KK}_{\mathrm{G}}\left(\mathrm{A}, \mathrm{C}_{0}(\mathrm{TN})\right) \stackrel{\left(\mathrm{k}_{\mathrm{TN}}^{\mathrm{TM}}\right)_{*}}{\longrightarrow} \mathrm{KK}_{\mathrm{G}}\left(\mathrm{A}, \mathrm{C}_{0}(\mathrm{TM})\right) .
$$

Here $\beta_{N} \in K_{K}(T S, T N)$ is the Bott element, as in Definition 4.6 .

We also have the usual map

$$
\left(j_{\mathrm{TS}}^{\mathrm{TM}}\right)^{*}: \mathrm{KK}_{\mathrm{G}}\left(\mathrm{A}, \mathrm{C}_{\mathrm{O}}(\mathrm{TM})\right) \rightarrow \mathrm{KK}_{\mathrm{G}}\left(\mathrm{A}, \mathrm{C}_{\mathrm{O}}(\mathrm{TS})\right) .
$$

Lemma 5.2. The map

$$
\left(j_{\mathrm{TS}}^{\mathrm{TM}}\right)^{*} \circ\left(\mathrm{j}_{\mathrm{TS}}^{\mathrm{TM}}\right)_{!}: \mathrm{KK}_{\mathrm{G}}\left(\mathrm{A}, \mathrm{C}_{\mathrm{o}}(\mathrm{TS})\right) \rightarrow \mathrm{KK}_{\mathrm{G}}\left(\mathrm{A}, \mathrm{C}_{0}(\mathrm{TS})\right)
$$

is given by the Kasparov product from the right with

$$
\left(j_{T S}^{\mathrm{TN}}\right)^{*} \beta_{\mathrm{N}} \in \mathrm{KK}_{\mathrm{G}}\left(\mathrm{C}_{0}(\mathrm{TS}), \mathrm{C}_{0}(\mathrm{TS})\right) \text {. }
$$

Proof. For all $x \in \mathrm{KK}_{\mathrm{G}}\left(\mathrm{A}, \mathrm{C}_{0}(\mathrm{TS})\right)$, functoriality of the Kasparov product implies that

$$
\begin{aligned}
\left(j_{\mathrm{TS}}^{\mathrm{TM}}\right)^{*} \circ\left(j_{\mathrm{TS}}^{\mathrm{TM}}\right)_{!}(x) & =\left(j_{\mathrm{TS}}^{\mathrm{TM}}\right)^{*} \circ\left(k_{\mathrm{TN}}^{\mathrm{TM}}\right)_{*}\left(x \otimes_{\mathrm{C}_{\mathrm{o}}(\mathrm{TS})} \beta_{\mathrm{N}}\right) \\
& =x \otimes_{\mathrm{C}_{0}(\mathrm{TS})}\left(\left(j_{\mathrm{TS}}^{\mathrm{TM}}\right)^{*} \circ\left(k_{\mathrm{TN}}^{\mathrm{TM}}\right)_{*} \beta_{\mathrm{N}}\right) .
\end{aligned}
$$

Since $\left(j_{T S}^{T M}\right)^{*} \circ\left(k_{T N}^{T M}\right)_{*}=\left(j_{T S}^{T N}\right)^{*}$, the claim follows.

Lemma 5.3. For any $\mathrm{G}$-invariant closed subset $\mathrm{X} \subset \mathrm{M}$, and any $\mathrm{G}$-invariant neighbourhood $\mathrm{V}$ of $\mathrm{X}$, the following diagram commutes:

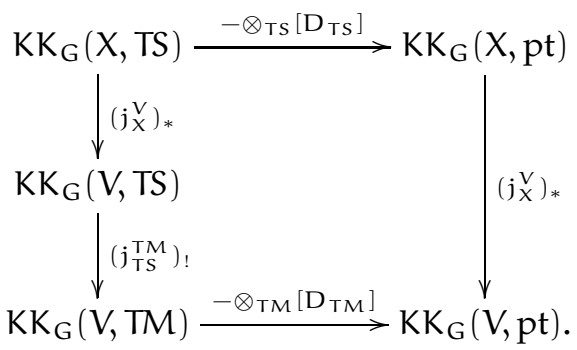

Proof. For all $a \in \mathrm{KK}_{\mathrm{G}}(\mathrm{X}, \mathrm{TS})$, functoriality and associativity of the Kasparov product imply that

$$
\begin{aligned}
\left(\left(j_{\mathrm{TS}}^{\mathrm{TM}}\right)_{!} \circ\left(j_{\mathrm{X}}^{\mathrm{V}}\right)_{*}(\mathrm{a})\right) \otimes_{\mathrm{TM}}\left[\mathrm{D}_{\mathrm{TM}}\right] & =\left(k_{\mathrm{TN}}^{\mathrm{TM}}\right)_{*}\left(\left(j_{\mathrm{X}}^{\mathrm{V}}\right)_{*}(\mathrm{a}) \otimes_{\mathrm{TS}} \beta_{\mathrm{N}}\right) \otimes_{\mathrm{TM}}\left[\mathrm{D}_{\mathrm{TM}}\right] \\
& =\left(j_{\mathrm{X}}^{\mathrm{V}}\right)_{*}(\mathrm{a}) \otimes_{\mathrm{TS}}\left(\left(k_{\mathrm{TN}}^{\mathrm{TM}}\right)_{*}\left(\beta_{\mathrm{N}}\right) \otimes_{\mathrm{TM}}\left[\mathrm{D}_{\mathrm{TM}}\right]\right) .
\end{aligned}
$$

$\operatorname{Now}\left(k_{\mathrm{TN}}^{\mathrm{TM}}\right)^{*}\left[\mathrm{D}_{\mathrm{TM}}\right]=\left[\mathrm{D}_{\mathrm{TN}}\right]$, so

$$
\begin{aligned}
\left(k_{\mathrm{TN}}^{\mathrm{TM}}\right)_{*}\left(\beta_{\mathrm{N}}\right) \otimes_{\mathrm{TM}}\left[\mathrm{D}_{\mathrm{TM}}\right] & =\beta_{\mathrm{N}} \otimes_{\mathrm{TN}}\left(k_{\mathrm{TN}}^{\mathrm{TM}}\right)^{*}\left[\mathrm{D}_{\mathrm{TM}}\right] \\
& =\beta_{\mathrm{N}} \otimes_{\mathrm{TN}}\left[\mathrm{D}_{\mathrm{TN}}\right] \\
& =\left[\mathrm{D}_{\mathrm{TS}}\right],
\end{aligned}
$$

where the last equality was proved in Proposition 4.7 


\subsection{Localisation and Gysin maps}

Let $\mathrm{U}$ and $\mathrm{V}$ be as in Theorem 2.3 . Consider the diagram

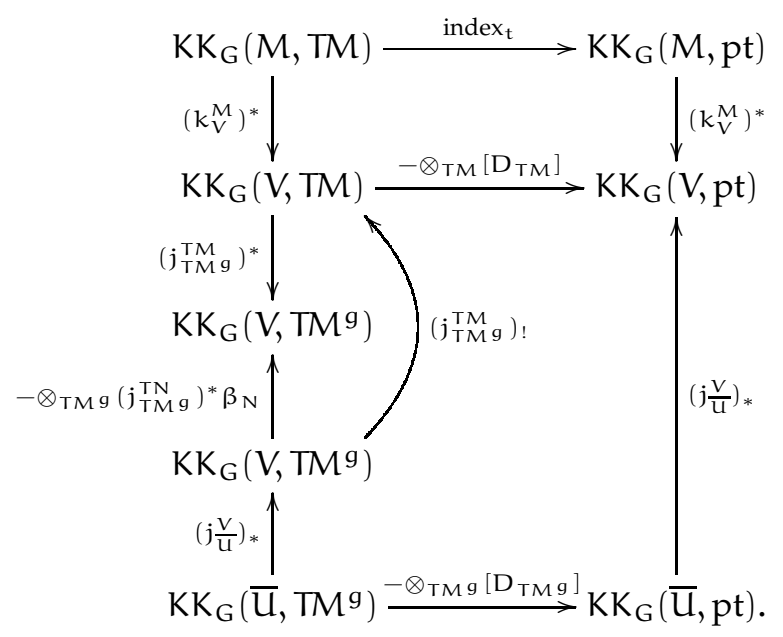

The top part of this diagram commutes because of functoriality of the Kasparov product. The part with the product with $\left(j_{\mathrm{TM}^{9}}^{\mathrm{TM}}\right) * \beta_{\mathrm{N}}$ in it commutes by Lemma 5.2, applied with $A=C_{0}(V)$, and $S$ running over the connected components of $\mathrm{M}^{\mathrm{g}}$. The remaining part of the diagram commutes by Lemma 5.3 , applied in a similar way with $S$ a connected component of $M^{g}$, and $X=\bar{U}$.

Diagram (5.1) can be extended as follows.

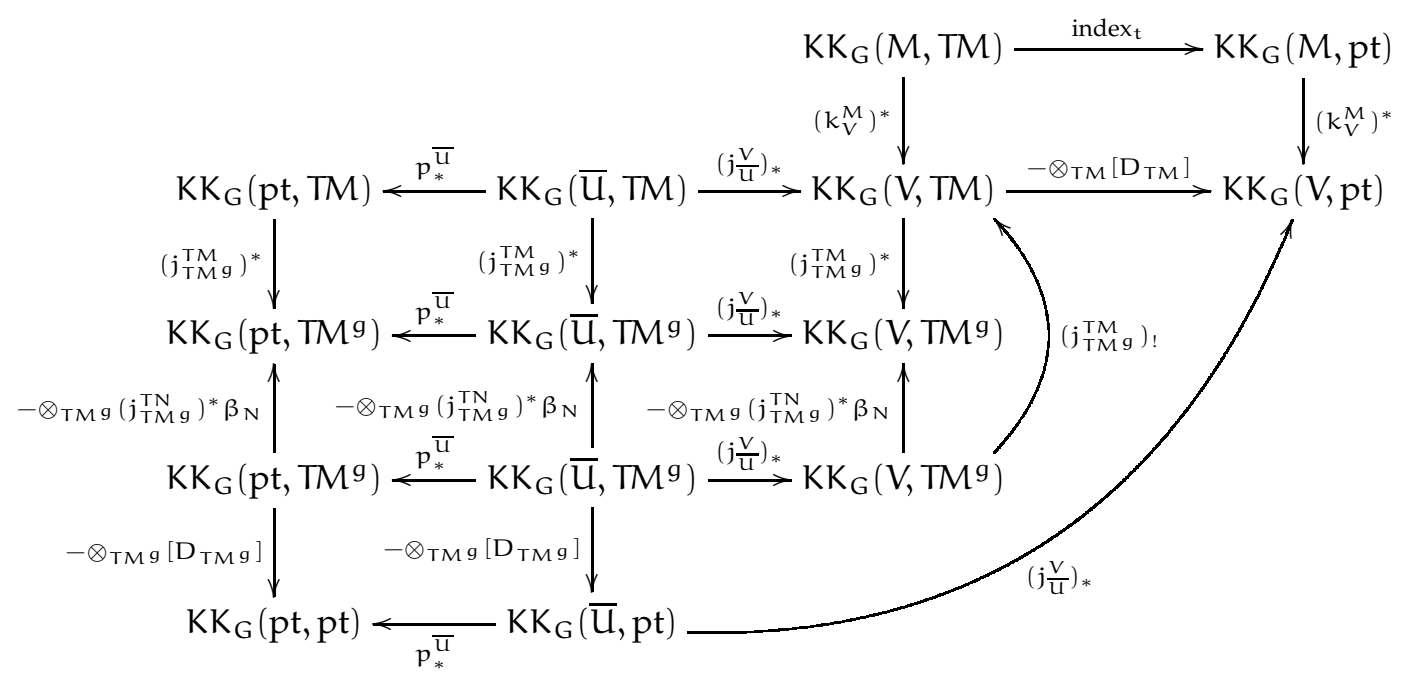

The right hand part of this diagram is diagram (5.1), and hence commutes. The other parts commute by functoriality of KK-theory and the Kasparov product. 
Theorem 2.3 implies that the maps $\left(j \frac{\mathrm{V}}{\mathrm{u}}\right)_{*}$ become invertible after localisation at $\mathrm{g}$. We will also use inverses of the localised classes

$$
\left(\left(j_{\mathrm{TM} g}^{\mathrm{TN}}\right)^{*} \beta_{\mathrm{N}}\right)_{\mathrm{g}} \in \mathrm{KK}_{\mathrm{G}}\left(\mathrm{TM}^{\mathrm{g}}, \mathrm{TM}^{\mathrm{g}}\right)_{\mathrm{g}}
$$

Lemma 5.4. The element (5.3) is invertible.

Proof. By Lemma 4.10, we have

$$
\left(j_{\mathrm{TM} g}^{\mathrm{TN}}\right)^{*} \beta_{\mathrm{N}}=\left[\tau_{M_{9}}^{*} \wedge \mathrm{N}_{\mathbb{C}}\right] .
$$

Atiyah and Segal showed in [4, Lemma 2.7] that $\left[\Lambda N_{\mathbb{C}}\right]$ is invertible in $K_{G}^{0}\left(M^{g}\right)_{g}$. The map

$$
\tau_{M^{g}}^{*}: K_{G^{\prime}}^{0}\left(M^{g}\right) \rightarrow K^{G}\left(\mathrm{TM}^{g}, \mathrm{TM}^{\mathrm{g}}\right)
$$

sending a class $[E] \in \mathrm{K}_{\mathrm{G}^{\prime}}^{0}\left(\mathrm{M}^{9}\right)$ to $\left[\tau_{\mathrm{M}^{g}}^{*} \mathrm{E}\right]$, is a unital ring homomorphism. Hence so is its localisation at $\mathrm{g}$. Therefore, the class

$$
\left[\tau_{M^{g}}^{*} \wedge N_{\mathbb{C}}\right]_{g}=\left(\tau_{M^{g}}^{*}\right)_{g}\left[\wedge N_{\mathbb{C}}\right]_{g} \quad \in K_{K_{G}}\left(\mathrm{TM}^{g}, \mathrm{TM}^{\mathrm{g}}\right)_{\mathrm{g}}
$$

is invertible.

\subsection{Evaluation}

Let $X$ and $Y$ be locally compact Hausdorff spaces with trivial actions by a compact group $\mathrm{G}$. Then the exterior Kasparov product

$$
\mathrm{KK}(\mathrm{X}, \mathrm{Y}) \times \mathrm{KK}_{\mathrm{G}}(\mathrm{pt}, \mathrm{pt}) \rightarrow \mathrm{KK}_{\mathrm{G}}(\mathrm{X}, \mathrm{Y})
$$

defines an isomorphism

$$
K K(X, Y) \otimes R(G) \cong K_{G}(X, Y) .
$$

If $\mathrm{X}$ is a point, this is a classical fact. We will also apply this isomorphism to the class $\left[\mathrm{D}_{\mathrm{TM}^{\mathrm{g}}}\right] \in \mathrm{KK}_{\mathrm{G}}\left(\mathrm{TM}^{\mathrm{g}}, \mathrm{pt}\right)$. There it is trivial, since $\mathrm{G}$ acts trivially on the Hilbert space in question. In the only other case we will use the isomorphism (5.4), we have $X=Y$, and this space has finitely many connected components. (To be precise, we will have $X=Y=\mathrm{TM}^{\mathrm{g}}$.) Let us work out the isomorphism explicitly in that case, for the cycles we will apply it to. These are G-equivariant Kasparov $\left(C_{0}(X), C_{0}(X)\right)$-cycles of the form $\left(\Gamma_{0}(E), F, \pi\right)$, where $\mathrm{E} \rightarrow \mathrm{X}$ is a vector bundle (of finite rank). Let $\mathrm{a} \in \mathrm{KK}_{\mathrm{G}}(\mathrm{X}, \mathrm{X})$ be the class of $\mathrm{a}$ cycle of this form, and let $b \in K K(X, X)$ be the class defined by the same cycle, where the group action is ignored. As $G$ acts trivially on $X$, each fibre of $E$ is a representation space of $G$. Suppose for simplicity that $X$ is connected; the general case follows by applying the arguments to its connected components. (This works since there are finitely many of them.) Since $X$ is connected, the representations by $\mathrm{G}$ on all fibres of $\mathrm{E}$ are equivalent. Let $\mathrm{V}$ be any one of these fibres, viewed as a representation space of $G$. Denote by $1_{G}$ the ring identity 
of $R(G)$, i.e. the trivial representation of $G$. Let $E_{0}:=X \times V \rightarrow X$ be the trivial bundle with fibre $\mathrm{V}$. Consider the representations

$$
\begin{aligned}
\pi_{X}^{X}: C_{0}(X) & \rightarrow \mathcal{B}\left(C_{0}(X)\right) ; \\
\pi_{X}^{E_{0}}: C_{0}(X) & \rightarrow \mathcal{B}\left(\Gamma_{0}\left(E_{0}\right)\right)
\end{aligned}
$$

defined by pointwise multiplication. Then

$$
\left(\left[\mathrm{C}_{0}(\mathrm{X}), 0, \pi_{\mathrm{X}}^{\mathrm{X}}\right] \otimes[\mathrm{V}]\right)+\left(\mathrm{b} \otimes 1_{\mathrm{G}}\right)=\left(\left[\Gamma_{0}\left(\mathrm{E}_{0}\right), 0, \pi_{\mathrm{X}}^{\mathrm{E}_{0}}\right] \otimes 1_{\mathrm{G}}\right)+\mathrm{a} \quad \in \mathrm{KK}_{\mathrm{G}}(\mathrm{X}, \mathrm{X}) .
$$

In fact, both sides of (5.5) are represented by the cycle

$$
\left(\Gamma_{0}\left(\mathrm{E}_{0} \oplus \mathrm{E}\right), 0 \oplus \mathrm{F}, \pi_{\mathrm{X}}^{\mathrm{E}_{0}} \oplus \pi\right),
$$

but, initially, with different G-actions. Namely, for the left-hand side of (5.5), G acts on the first summand $E_{0}$ in (5.6), while for the right-hand side of (5.5), G acts on the second summand $E$ in (5.6). As $G$ acts trivially on $X$, representations of $\mathrm{G}$ commute with those of $\mathrm{C}_{0}(\mathrm{X})$. Since, in addition, $\mathrm{F}$ is $\mathrm{G}$-invariant, these two actions by $\mathrm{G}$ can be connected by a rotation homotopy, so (5.5) follows. In that equality, $a$ is represented as an element of $K K(X, Y) \otimes R(G)$.

In general, using (5.4), one can apply the evaluation $\mathrm{ev}_{\mathrm{g}}=1 \otimes \mathrm{ev}_{\mathrm{g}}$ as a map

$$
\mathrm{ev}_{\mathrm{g}}: \mathrm{KK}_{\mathrm{G}}(\mathrm{X}, \mathrm{Y}) \rightarrow \mathrm{KK}(\mathrm{X}, \mathrm{Y}) \otimes \mathbb{C} .
$$

This map is compatible with localisation at $\mathrm{g}$, in the sense that the following diagram commutes:

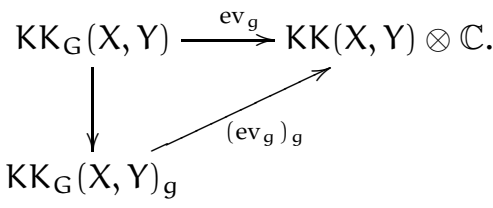

If $a \in K_{G}(X, Y)$, we will also write

$$
\mathrm{a}(\mathrm{g}):=\mathrm{ev}_{\mathrm{g}}(\mathrm{a}) \quad \in \mathrm{KK}(\mathrm{X}, \mathrm{Y}) \otimes \mathbb{C} .
$$

The evaluation map (5.7) is compatible with Kasparov products. This follows from the facts that the isomorphism (5.4) is compatible with the product, that Kasparov products in $R(G)$ coincide with tensor products of representations, and that the character of the tensor product of two finite-dimensional representations is the product of the characters of the individual representations.

Hence we can attach the following commutative diagram to the lower left 
hand side of (5.2):

$$
\begin{aligned}
& \mathrm{KK}\left(\mathrm{pt}, \mathrm{TM}^{\mathrm{g}}\right) \otimes \mathbb{C} \stackrel{\mathrm{ev}_{\mathrm{g}}}{\longleftarrow} \mathrm{KK}_{\mathrm{G}}\left(\mathrm{pt}, \mathrm{TM}^{\mathrm{g}}\right) \\
& \left(-\otimes_{T M g}\left(j_{T M}^{T M}\right)^{*} \beta_{N}(g)\right) \otimes 1 \uparrow \quad-\otimes_{T M g}\left(j_{T M}^{T N}\right)^{*} \beta_{N} \uparrow \\
& \mathrm{KK}\left(\mathrm{pt}, \mathrm{TM}^{\mathrm{g}}\right) \otimes \mathbb{C} \stackrel{\mathrm{ev}_{g}}{\longleftarrow} \mathrm{KK}_{\mathrm{G}}\left(\mathrm{pt}, \mathrm{TM}^{\mathrm{g}}\right) \\
& \left(-\otimes_{\text {TM }}\left[D_{\text {TM } g}\right]\right) \otimes 1 \mid
\end{aligned}
$$

Here, $\left[D_{\mathrm{TM}^{g}}\right] \in \mathrm{KK}\left(\mathrm{TM}^{\mathrm{g}}, \mathrm{pt}\right)$ is identified with $\left[\mathrm{D}_{\mathrm{TM}^{\mathrm{g}}}\right] \otimes 1 \in \mathrm{KK}\left(\mathrm{TM}^{\mathrm{g}}, \mathrm{pt}\right) \otimes$ $R(G)$, so that $e_{g}\left(\left[D_{T_{M} g}\right]\right)=\left[D_{T_{M} g}\right] \otimes 1$. In particular, when $M^{g}=p t$, the vertical map on the lower left corner is the identity.

By Lemma 4.11 and compactness of $M^{9}$, the map

$$
-\otimes_{\mathrm{TM}^{g}}\left[\mathrm{D}_{\mathrm{TM}^{\mathrm{g}}}\right]: \mathrm{KK}\left(\mathrm{pt}, \mathrm{TM}^{\mathrm{g}}\right) \rightarrow \mathrm{KK}(\mathrm{pt}, \mathrm{pt})
$$

is the Atiyah-Singer topological index map index $x_{t}^{A S}$. We will use the same notation for its extension to a map KK $\left(\mathrm{pt}, \mathrm{TM}^{\mathrm{g}}\right) \otimes \mathbb{C} \rightarrow \mathbb{C}$.

Using commutativity of (5.2) and (5.8), and invertibility of the localised maps $\left(\left(j \frac{V}{\mathrm{U}}\right)_{*}\right)_{g}$ and classes (5.3), we obtain the commutative diagram

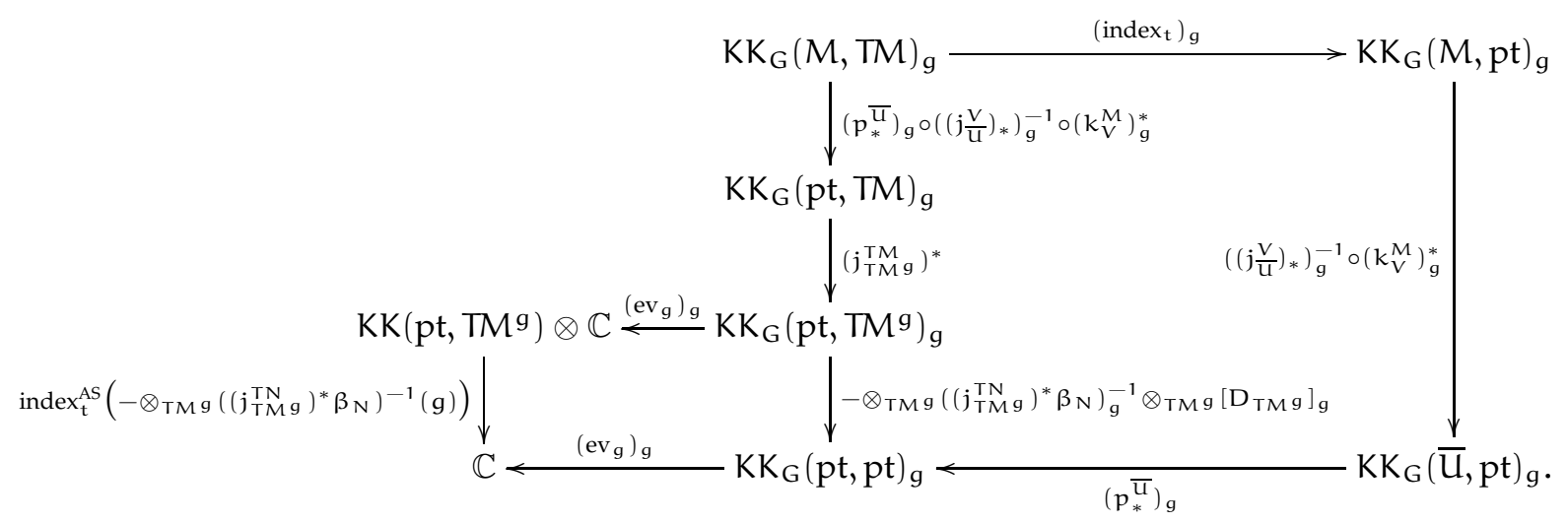

\subsection{The g-symbol class}

Recall that in (4.3) we defined the class

$$
\left[\sigma_{\mathrm{D}}\right] \in \mathrm{KK}_{\mathrm{G}}(\mathrm{M}, \mathrm{TM}) .
$$

The last ingredient of the proof of Theorem 2.9 is a class defined by $\sigma_{\mathrm{D}}$ in the topological K-theory of TM, localised at $\mathrm{g}$. In Section 7 we will describe this class more explicitly, and use it to obtain another expression for the $\mathrm{g}$-index. 
Definition 5.5. The g-symbol class class of $\mathrm{D}$ is the class $\sigma_{g}^{\mathrm{D}}$ in the localised topological K-theory of TM defined by

$$
\sigma_{g}^{\mathrm{D}}:=\left(\mathrm{p}_{*}^{\overline{\mathrm{u}}}\right)_{\mathrm{g}} \circ\left(\left(\mathrm{j} \frac{\mathrm{V}}{\mathrm{U}}\right)_{*}\right)_{\mathrm{g}}^{-1} \circ\left(\mathrm{k}_{\mathrm{V}}^{\mathrm{M}}\right)_{\mathrm{g}}^{*}\left[\sigma_{\mathrm{D}}\right]_{\mathrm{g}} \quad \in \mathrm{KK}_{\mathrm{G}}(\mathrm{pt}, \mathrm{TM})_{\mathrm{g}} .
$$

The g-symbol class generalises the usual symbol class in the compact case.

Lemma 5.6. If $\mathrm{M}$ is compact, then $\sigma_{\mathrm{g}}^{\mathrm{D}}$ is the localisation at $\mathrm{g}$ of the usual class of $\sigma_{\mathrm{D}}$ in $\mathrm{KK}_{\mathrm{G}}(\mathrm{pt}, \mathrm{TM})$.

Proof. If $M$ is compact, then we can choose $U=V=M$. Then, since the map $\mathrm{p}^{M}: M \rightarrow \mathrm{pt}$ is proper, we have

$$
\sigma_{g}^{\mathrm{D}}=\left(\mathrm{p}_{*}^{\mathrm{M}}\left[\sigma_{\mathrm{D}}\right]\right)_{\mathrm{g}},
$$

which is the usual symbol class by Lemma 4.2 .

We now prove some properties of the g-symbol class that will be used in the proof of Theorem 2.9] As before, we write $\tilde{\sigma}_{D}:=\frac{\sigma_{D}}{\sqrt{\sigma_{D}^{2}+1}}$.

Lemma 5.7. The class

$$
\left(k_{V}^{M}\right)_{g}^{*}\left[\sigma_{D}\right]_{g} \in K_{G}(V, T M)_{g}
$$

is the localisation at $\mathrm{g}$ of the class

$$
\left[\left.\sigma_{\mathrm{D}}\right|_{\mathrm{V}}\right]_{\mathrm{TM}}:=\left[\Gamma_{\mathrm{O}}\left(\tau_{\mathrm{V}}^{*}\left(\left.\mathrm{E}\right|_{\mathrm{V}}\right)\right),\left.\tilde{\sigma}_{\mathrm{D}}\right|_{\mathrm{TV}}, \pi_{\mathrm{V}}\right] \in \mathrm{KK}_{\mathrm{G}}(\mathrm{V}, \mathrm{TM}) .
$$

Here the $\mathrm{C}_{0}(\mathrm{TM})$ valued inner product on $\Gamma_{0}\left(\left.\mathrm{E}\right|_{V}\right)$ is defined by the natural $\mathrm{C}_{0}(\mathrm{TV})$ valued inner product, composed with the inclusion $\mathrm{k}_{\mathrm{TV}}^{\mathrm{TM}}$.

Proof. The class

$$
\left(k_{V}^{M}\right)^{*}\left[\sigma_{D}\right] \in \mathrm{KK}_{\mathrm{G}}(\mathrm{V}, \mathrm{TM})
$$

is represented by the Kasparov cycle

$$
\begin{aligned}
\left(\Gamma_{0}\left(\tau_{M}^{*} E\right), \tilde{\sigma}_{D},\left(k_{V}^{M}\right)^{*} \pi_{M}\right)=\left(\Gamma _ { 0 } \left(\tau_{V}^{*}\left(\left.E\right|_{V}\right)\right.\right. & \left.,\left.\tilde{\sigma}_{D}\right|_{T V}, \pi_{V}\right) \\
& \oplus\left(\Gamma_{0}\left(\tau_{M \backslash V}^{*}\left(\left.E\right|_{M \backslash V}\right)\right),\left.\tilde{\sigma}_{D}\right|_{T M \backslash T V}, 0\right) .
\end{aligned}
$$

The second term on the right hand side is a degenerate cycle, so the claim follows.

Consider the class

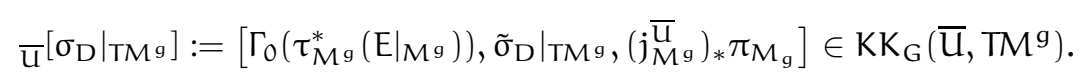

Lemma 5.8. We have

$$
\left(j \frac{\mathrm{V}}{\mathrm{U}}\right)_{*}\left(\overline{\mathrm{u}}\left[\left.\sigma_{\mathrm{D}}\right|_{\mathrm{TM}^{g}}\right]\right)=\left(j_{\mathrm{TM}^{\mathrm{g}}}^{\mathrm{TM}}\right)^{*}\left[\left.\sigma_{\mathrm{D}}\right|_{\mathrm{V}}\right]_{\mathrm{TM}} \in \mathrm{KK}_{\mathrm{G}}\left(\mathrm{V}, \mathrm{TM}^{\mathrm{g}}\right) .
$$


Proof. By definition,

$$
\left(j_{T_{M} g}^{T M}\right)^{*}\left[\left.\sigma_{D}\right|_{V}\right]_{T M}=\left[\Gamma_{0}\left(\tau_{V}^{*}\left(\left.E\right|_{V}\right)\right) \otimes_{j_{T M}^{T M}}^{T M} C_{0}\left(T^{g}\right),\left.\tilde{\sigma}_{D}\right|_{V} \otimes 1, \pi_{V} \otimes 1\right] .
$$

The map

$$
\Gamma_{0}\left(\tau_{V}^{*}\left(\left.E\right|_{V}\right)\right) \otimes_{j_{T M}^{T M}} C_{0}\left(T^{g}\right) \rightarrow \Gamma_{0}\left(\tau_{M^{g}}^{*}\left(\left.E\right|_{M^{g}}\right)\right)
$$

that maps $s \otimes \varphi$ to $\left.\varphi s\right|_{\mathrm{TM}^{g}}$, for $s \in \Gamma_{0}\left(\tau_{V}^{*}\left(\left.\mathrm{E}\right|_{V}\right)\right)$ and $\varphi \in \mathrm{C}_{0}\left(\mathrm{TM}^{g}\right)$, is an isomorphism of Hilbert $C_{0}\left(\mathrm{TM}^{\mathrm{g}}\right)$-modules. It intertwines the operators $\left.\tilde{\sigma}_{\mathrm{D}}\right|_{V} \otimes 1$ and $\left.\tilde{\sigma}_{\mathrm{D}}\right|_{T_{M} g}$, and the representations $\pi_{V} \otimes 1$ and

$$
\left(j_{M^{g}}^{V}\right)_{*} \pi_{M^{g}}=\left(j \frac{V}{U}\right)_{*}\left(j_{M^{g}}^{\bar{u}}\right)_{*} \pi_{M^{g}} .
$$

The lemma is then proved.

Proposition 5.9. The class

$$
\left(j_{\mathrm{TM}^{g}}^{\mathrm{TM}}\right)_{\mathrm{g}}^{*} \sigma_{g}^{\mathrm{D}} \in \mathrm{KK} K_{\mathrm{G}}\left(\mathrm{pt}, \mathrm{TM}^{\mathrm{g}}\right)_{\mathrm{g}}
$$

is the localisation at $\mathrm{g}$ of the usual class $\left[\left.\sigma_{\mathrm{D}}\right|_{\mathrm{TM}^{\mathrm{g}}}\right]$ in the equivariant topological $\mathrm{K}$ theory of $\mathrm{TM}^{\mathrm{g}}$.

Proof. By commutativity of (the top left part of) diagram (5.2), we have

$$
\left(j_{\mathrm{TM}^{g}}^{\mathrm{TM}}\right)_{\mathrm{g}}^{*} \sigma_{\mathrm{g}}^{\mathrm{D}}=\left(\mathrm{p}_{*}^{\overline{\mathrm{u}}}\right)_{\mathrm{g}} \circ\left(\left(j \frac{\mathrm{V}}{\mathrm{u}}\right)_{*}\right)_{\mathrm{g}}^{-1} \circ\left(j_{\mathrm{TM}}^{\mathrm{TM}}\right)_{\mathrm{g}}^{*} \circ\left(k_{\mathrm{V}}^{\mathrm{M}}\right)_{\mathrm{g}}^{*}\left[\sigma_{\mathrm{D}}\right]_{\mathrm{g}} .
$$

By Lemma 5.7, we have

$$
\left(k_{V}^{M}\right)_{g}^{*}\left[\sigma_{D}\right]_{g}=\left(\left[\sigma_{D} \mid v\right]_{T M}\right)_{g} .
$$

By Lemma 5.8 we have

$$
\left(\left(j \frac{\mathrm{V}}{\mathrm{U}}\right)_{*}\right)_{\mathrm{g}}^{-1} \circ\left(j_{\mathrm{TM}^{g}}^{\mathrm{TM}}\right)_{\mathrm{g}}^{*}\left(\left[\left.\sigma_{\mathrm{D}}\right|_{\mathrm{V}}\right]_{\mathrm{TM}}\right)_{g}=\overline{\mathrm{u}}\left[\left.\sigma_{\mathrm{D}}\right|_{\mathrm{TM}^{g}}\right]_{g} .
$$

By Lemma 4.2, we have

$$
p_{*}^{\bar{U}}\left(\overline{\mathrm{u}}\left[\left.\sigma_{\mathrm{D}}\right|_{\mathrm{TM}^{g}}\right]\right)=\left[\left.\sigma_{\mathrm{D}}\right|_{\mathrm{TM}}\right] \quad \in \mathrm{KK}_{\mathrm{G}}\left(\mathrm{pt}, \mathrm{TM}^{\mathrm{g}}\right) .
$$

So the claim follows.

We have now finished all preparation needed to prove Theorem 2.9

Proof of Theorem 2.9] Using Kasparov's index theorem, Theorem 4.3 , and commutativity of (5.9), we find that

$$
\begin{aligned}
\operatorname{index}_{g}(D) & =\left(\operatorname{ev}_{g}\right)_{g} \circ\left(p_{*}^{\bar{u}}\right)_{g} \circ\left(\left(j \frac{V}{U}\right)_{*}\right)_{g}^{-1} \circ\left(k_{V}^{M}\right)_{g}^{*}[D] \\
& =\left(\operatorname{ev}_{g}\right)_{g} \circ\left(p_{*}^{\bar{u}}\right)_{g} \circ\left(\left(j \frac{V}{U}\right)_{*}\right)_{g}^{-1} \circ\left(k_{V}^{M}\right)_{g}^{*} \circ\left(\operatorname{index}_{t}\right)_{g}\left[\sigma_{D}\right]_{g} \\
& =\operatorname{index}_{t}^{A S}\left(\left(\left(j M_{T^{g}}^{\mathrm{TM}}\right)^{*} \sigma_{g}^{D}\right)(g) \otimes_{T^{g}}\left(\left(j_{T_{M} g}^{T N}\right)^{*} \beta_{N}\right)^{-1}(g)\right) .
\end{aligned}
$$

By Lemma 4.10 and Proposition [5.9, the latter expression equals

$$
\operatorname{index}_{t}^{A S}\left(\left[\left.\sigma_{D}\right|_{T_{M} g}\right](g) \otimes_{T_{M} g}\left[\Lambda N_{\mathbb{C}}\right]^{-1}(g)\right) .
$$


Furthermore,

$$
\left[\left.\sigma_{D}\right|_{T_{M g}}\right](g) \otimes_{T_{M} g}\left[\tau_{M^{g}}^{*} \wedge N_{\mathbb{C}}\right]^{-1}(g)=\left[\left.\sigma_{D}\right|_{T_{M} g}\right](g) \cdot\left[\Lambda N_{\mathbb{C}}\right]^{-1}(g),
$$

where the dot means the right $\mathrm{K}_{\mathrm{G}}^{0}\left(\mathrm{M}^{\mathrm{g}}\right)$-module structure of $\mathrm{K}_{\mathrm{G}}^{0}\left(\mathrm{TM}^{\mathrm{g}}\right)$. We conclude that

$$
\operatorname{index}_{g}(D)=\operatorname{index}_{t}^{A S}\left(\left[\left.\sigma_{D}\right|_{T M g}\right](g) \cdot\left[\wedge N_{\mathbb{C}}\right]^{-1}(g)\right) .
$$

Theorem 2.9 now follows from the definition of the topological index map (4.15), and multiplicativity of the Chern character.

\subsection{The index pairing}

The arguments used to prove Theorem 2.9 also imply Theorem 2.10 about the index pairing. In fact, the parts of the proof of Theorem 2.9 about localisation in the first entry of KK-theory are not needed in the proof Theorem 2.10 .

The key step is a localisation property of the K-homology class of D, localised at $\mathrm{g}$.

Proposition 5.10. We have

$$
[\mathrm{D}]_{\mathrm{g}}=\left(j_{\mathrm{TM}^{\mathrm{g}}}^{\mathrm{TM}}\right)_{\mathrm{g}}^{*}\left[\sigma_{\mathrm{D}}\right]_{\mathrm{g}} \otimes_{\mathrm{TM}^{g}}\left[\tau_{\mathrm{M}^{g}}^{*} \wedge \mathrm{N}_{\mathbb{C}^{-}}\right]_{\mathrm{g}}^{-1} \otimes_{\mathrm{TM}^{g}}\left[\mathrm{D}_{\mathrm{TM}^{g}}\right]_{\mathrm{g}} \quad \in \mathrm{KK}_{\mathrm{G}}(\mathrm{M}, \mathrm{pt})_{\mathrm{g}} .
$$

Proof. Lemmas 5.2 and 5.3 imply that the following diagram commutes:

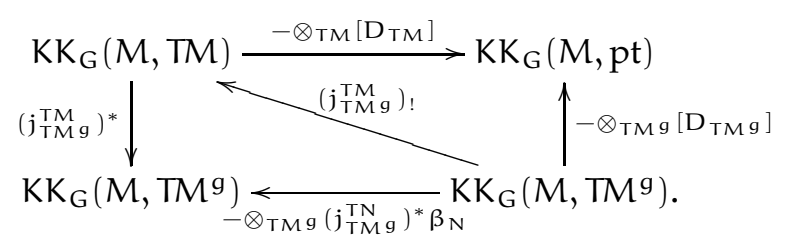

Therefore, the claim follows from Lemmas 4.10 and 5.4 , and Theorem 4.3

Proof of Theorem 2.10 Let $[\mathrm{F}] \in \mathrm{KK}_{\mathrm{G}}(\mathrm{pt}, \mathrm{M})$ be as in Subsection 2.5. By compatibility of the Kasparov product with localisation and evaluation, Proposition 5.10 implies that

$$
\begin{aligned}
\left([\mathrm{F}] \otimes_{\mathrm{M}}[\mathrm{D}]\right)(\mathrm{g}) & =\left([\mathrm{F}]_{\mathrm{g}} \otimes_{\mathrm{M}}[\mathrm{D}]_{\mathrm{g}}\right)(\mathrm{g}) \\
& =\left([\mathrm{F}]_{\mathrm{g}} \otimes_{\mathrm{M}}\left(j_{\mathrm{TM}^{g}}^{\mathrm{TM}}\right)_{\mathrm{g}}^{*}\left[\sigma_{\mathrm{D}}\right]_{\mathrm{g}}\right)(\mathrm{g}) \otimes_{\mathrm{TM}^{g}}\left[\tau_{\mathrm{M}^{g}}^{*} \wedge \mathrm{N}_{\mathbb{C}}\right](g)^{-1} \otimes_{\mathrm{TM}^{g}}\left[\mathrm{D}_{\mathrm{TM}^{g}}\right](\mathrm{g}) .
\end{aligned}
$$

Now

$$
\left([\mathrm{F}]_{\mathrm{g}} \otimes_{\mathrm{M}}\left(\mathrm{j}_{\mathrm{TM}^{\mathrm{g}}}^{\mathrm{TM}}\right)_{\mathrm{g}}^{*}\left[\sigma_{\mathrm{D}}\right]_{\mathrm{g}}\right)(\mathrm{g})=\left[\tau_{M^{g}}^{*}\left(\left.\mathrm{~F}\right|_{M^{g}}\right)\right](\mathrm{g}) \otimes\left[\left.\sigma_{\mathrm{D}}\right|_{\mathrm{TM}^{g}}\right](\mathrm{g}) \quad \in \mathrm{KK}\left(\mathrm{pt}, \mathrm{TM}^{\mathrm{g}}\right) \otimes \mathbb{C},
$$

where on the right hand side, the tensor product denotes the ring structure on the topological K-theory of $\mathrm{TM}^{\mathrm{g}}$. Therefore, and because $\left[\mathrm{D}_{\mathrm{TM}^{\mathrm{g}}}\right](\mathrm{g})=$ $\left[\mathrm{D}_{\mathrm{TM}^{\mathrm{g}}}\right] \otimes 1 \in \mathrm{KK}\left(\mathrm{TM}^{\mathrm{g}}, \mathrm{pt}\right) \otimes \mathbb{C}$, the claim follows from Lemma 4.11. 


\section{Examples and applications}

The g-index was defined in terms of KK-theory, but Theorem 2.9 allows us to express it entirely in cohomological terms. Using this theorem, we can compute the g-index explicitly in examples, and show how it is related to other indices.

For finite fixed point sets, Theorem 2.9 has a simpler form, as discussed in Subsection 6.1. In Subsection 6.2, we give a linearisation theorem for the $\mathrm{g}$-index of a twisted Dolbeault-Dirac operator on a complex manifold, in the case of a finite fixed point set. We then work out the example of the DolbeaultDirac operator on the complex plane, acted on by the circle, in Subsection 6.3 An illustration of the linearisation theorem is given in Subsection 6.4, where we apply it to the two-sphere, to decompose the usual equivariant index. In Subsection 6.5. we realise characters of discrete series representations of semisimple Lie groups on regular points of a maximal torus, in terms of the g-index. For Fredholm operators, and in particular Callias-type deformations of Dirac operators, we describe the relation between the g-index and the character of the action by $\mathrm{g}$ on the kernel of such an operator, in Subsection 6.6. We then give a relation with an index studied by Braverman in Subsection 6.7 and a relative index theorem along the lines of work by Gromov and Lawson in Subsection 6.8. In Subsection 6.9, we mention some geometric consequences of the vanishing or nonvanishing of the g-index of a Hodge-Dirac or Spin-Dirac operator.

\subsection{Finite fixed point sets}

If the fixed point set $\mathrm{M}^{\mathrm{g}}$ is 0 -dimensional, then $\mathrm{TM}^{\mathrm{g}}=\mathrm{M}^{\mathrm{g}}, \tau_{\mathrm{M}^{g}}$ is the identity map, $\operatorname{Todd}\left(\mathrm{TM}^{\mathrm{g}} \otimes \mathbb{C}\right)$ is trivial and

$$
\operatorname{ch}\left(\left[\left.\sigma_{\mathrm{D}}\right|_{\mathrm{TM}^{g}}\right](g)\right)=\operatorname{Tr}\left(\left.g\right|_{E^{+}}\right)-\operatorname{Tr}\left(\left.g\right|_{E^{-}}\right) .
$$

Furthermore, since $\mathrm{M}^{\mathrm{g}}$ only consists of isolated points, we have

$$
\mathrm{K}^{0}\left(M^{g}\right)=\bigoplus_{m \in M^{g}} \mathbb{Z}=H^{*}\left(M^{g}\right)
$$

and the Chern character is the identity map. So we now have, at a fixed point $\mathrm{m} \in \mathrm{M}^{\mathrm{g}}$,

$$
\operatorname{ch}\left(\left[\Lambda N_{\mathbb{C}}\right](g)\right)_{m}=\operatorname{ch}\left(\left[\left.\Lambda M_{\mathbb{C}^{\prime}}\right|_{M^{g}}\right](g)\right)_{m}=\operatorname{det}_{\mathbb{R}}\left(1-\left.g\right|_{T_{m} M}\right) .
$$

The last equality is obtained by evaluating the virtual character of $\wedge T_{m} M_{\mathbb{C}}$ at g, so one obtains

$$
\operatorname{Tr}_{\mathbb{C}}\left(\left.g\right|_{\bigwedge^{\text {even }} T_{m} M_{\mathbb{C}}}\right)-\operatorname{Tr}_{\mathbb{C}}\left(\left.g\right|_{\bigwedge^{\text {odd }}}{T_{m}}_{M_{\mathbb{C}}}\right) .
$$

Therefore, Theorem 2.9 implies the following generalisation of Atiyah-Bott's fixed point theorem [2, Theorem A] to noncompact manifolds, but for compact G. 
Corollary 6.1. When $\mathrm{M}^{\mathrm{g}}$ is a finite set of points,

$$
\operatorname{index}_{g}(D)=\sum_{m \in M^{g}} \frac{\operatorname{Tr}\left(\left.g\right|_{E_{m}^{+}}\right)-\operatorname{Tr}\left(\left.g\right|_{E_{m}^{-}}\right)}{\operatorname{det}_{\mathbb{R}}\left(1-\left.g^{-1}\right|_{T_{m} M}\right)} .
$$

Remark 6.2. In the statement of Atiyah-Bott fixed point theorem, the denominator is $\left|\operatorname{det}_{\mathbb{R}}\left(1-\left.g\right|_{T_{m} M}\right)\right|$. In our case, $g$ is contained in a compact group $G$, so the real eigenvalues of $g$ are 1 or -1 . Thus $\operatorname{det}_{\mathbb{R}}\left(1-\left.g^{-1}\right|_{T_{m} M}\right)$ is always positive. See also page 186 in [8]. Also, the fact that $g$ acts orthogonally on $T_{m} M$ implies that $\operatorname{det}_{\mathbb{R}}\left(1-\left.g^{-1}\right|_{T_{m} M}\right)=\operatorname{det}_{\mathbb{R}}\left(1-\left.g\right|_{T_{m} M}\right)$

Now suppose $M$ is a complex manifold, and suppose $g$ is holomorphic. Let $\mathrm{F} \rightarrow \mathrm{M}$ be a holomorphic vector bundle, and consider the Dolbeault-Dirac operator $\bar{\partial}_{\mathrm{F}}+\bar{\partial}_{\mathrm{F}}^{*}$ on $M$, coupled to $\mathrm{F}$.

Corollary 6.3. If $\mathrm{M}^{\mathrm{g}}$ is a finite set of points, then

$$
\operatorname{index}_{g}\left(\bar{\partial}_{\mathrm{F}}+\bar{\partial}_{\mathrm{F}}^{*}\right)=\sum_{m \in M^{g}} \frac{\operatorname{Tr}_{\mathbb{C}}\left(\left.g\right|_{\mathrm{F}_{m}}\right)}{\operatorname{det}_{\mathbb{C}}\left(1-\left.g^{-1}\right|_{T_{m} M}\right)} .
$$

For equivalent expressions, note that

$$
\operatorname{det}_{\mathbb{C}}\left(1-\left.g^{-1}\right|_{T_{m}^{1,0} M}\right)=\operatorname{det}_{\mathbb{C}}\left(1-\left.g^{-1}\right|_{T_{m} M}\right)=\operatorname{det}_{\mathbb{C}}\left(1-\left.g\right|_{T_{m}^{O, 1} M}\right)
$$

in (6.2).

Proof. In Theorem 4.12 of [2], it is shown that in this situation, the right hand side of (6.1) equals the right hand side of (6.2). The key observation is that the supertrace of $\left.g\right|_{\wedge^{*}\left(T^{0,1} M\right)}$ is cancelled by the second factor in

$$
\operatorname{det}_{\mathbb{R}}\left(1-\left.g^{-1}\right|_{T_{m} M}\right)=\operatorname{det}_{\mathbb{C}}\left(1-\left.g^{-1}\right|_{T_{m}^{1,0} M}\right) \operatorname{det}_{\mathbb{C}}\left(1-\left.g^{-1}\right|_{T_{m}^{o, 1} M}\right) .
$$

(See also [8, Corollary 6.8].)

\subsection{A holomorphic linearisation theorem}

A tool used in some index problems is a linearisation theorem, relating an index to indices on vector spaces. See for example Chapter 4 of [21] and Theorem 7.2 in [12]. A version for Callias-type operators can be decuced from Theorem 2.16 in [14]. In those references, cobordism arguments are used to prove linearisation theorems. We will use the excision property of the g-index to obtain an analogous result. (So we do not use Theorem[2.9 here.) We will state and prove this result in the setting of Corollary 6.3, where $M$ is a complex manifold, $D$ is the Dolbeault-Dirac operator coupled to a holomorphic vector bundle $F \rightarrow M$, and $M^{g}$ is finite. A more general statement, where $M^{9}$ is not finite or $D$ is not a Dolbeault-Dirac operator, is possible, but would be less explicit.

Under these assumptions, for any $m \in M^{g}$, let $\bar{\partial}^{T_{m}} M$ be the Dolbeault operator on the complex vector space $T_{m} M$. 
Corollary 6.4 (Holomorphic linearisation theorem). We have

$$
\operatorname{index}_{g}\left(\bar{\partial}_{F}+\bar{\partial}_{F}^{*}\right)=\sum_{m \in M} \operatorname{Tr}_{\mathbb{C}}\left(\left.g\right|_{F_{m}}\right) \operatorname{index}_{g}\left(\bar{\partial}^{T_{m} M}+\left(\bar{\partial}^{T_{m} M}\right)^{*}\right) .
$$

Proof. By Lemma 2.7, the g-index of $\bar{\partial}_{\mathrm{F}}+\bar{\partial}_{\mathrm{F}}^{*}$ equals the g-index of the DolbeaultDirac operator on the union over $m \in M^{g}$ of the tangent spaces $T_{m} M$, coupled to the vector bundle which on every space $T_{m} M$ is trivial with fibre $F_{m}$. It follows directly from the definition that the $\mathrm{g}$-index is additive with respect to disjoint unions. Hence

$$
\begin{aligned}
\operatorname{index}_{g}\left(\bar{\partial}_{\mathrm{F}}+\bar{\partial}_{\mathrm{F}}^{*}\right) & =\sum_{m \in M^{g}} \operatorname{index}_{g}\left(\bar{\partial}^{T_{m} M} \otimes 1_{F_{m}}+\left(\bar{\partial}^{T_{m} M}\right)^{*} \otimes 1_{F_{m}}\right) \\
& =\sum_{m \in M^{g}} \operatorname{Tr}_{\mathbb{C}}\left(\left.g\right|_{F_{m}}\right) \operatorname{index}_{g}\left(\bar{\partial}^{T_{m} M}+\left(\bar{\partial}^{T_{m} M}\right)^{*}\right) .
\end{aligned}
$$

An example on computing and explicitly realising an index of the form

$$
\operatorname{index}_{g}\left(\bar{\partial}^{T_{m} M}+\left(\bar{\partial}^{T_{m} M}\right)^{*}\right)
$$

as in Corollary 6.4 is given in the next subsection. An example showing that the linearisation theorem gives a natural result if $M$ is compact is given in Subsection 6.4

\subsection{The circle acting on the plane}

Consider the usual action by the circle $\mathbb{T}^{1}=U(1)$ on the complex plane $\mathbb{C}$, and the (untwisted) Dolbeault-Dirac operator $\bar{\partial}+\bar{\partial}^{*}$ on $\mathbb{C}$. We will compute the distribution $\Theta$ on $\mathbb{T}^{1}$ given by the function

$$
g \mapsto \operatorname{index}_{g}\left(\bar{\partial}+\bar{\partial}^{*}\right) .
$$

This function is defined on the set of elements $g \in \mathbb{T}^{1}$ with dense powers, i.e. the elements of the form $g=e^{\sqrt{-1} \alpha}$, where $\alpha \in \mathbb{R} \backslash 2 \pi \mathbb{Q}$. So the function is defined almost everywhere.

By Corollary 6.3, we have for such $g$,

$$
\operatorname{index}_{g}\left(\bar{\partial}+\bar{\partial}^{*}\right)=\frac{1}{1-g^{-1}}
$$

So the function (6.3) is given by $g \mapsto 1 /\left(1-g^{-1}\right)$ almost everywhere. One can deduce that the sum of functions

$$
\sum_{k=0}^{\infty}\left(g \mapsto g^{-k}\right)
$$

converges as a distribution on $\mathbb{T}^{1}$ to $\Theta$. 
This allows us to describe the g-index of $\bar{\partial}+\bar{\partial}^{*}$ in terms of its kernel. Indeed, consider the Euclidean density $\mathrm{d} z=\mathrm{d} x \mathrm{~d} y$ on $\mathbb{C}$, and the corresponding space $\mathrm{L}^{2}(\mathbb{C})$. Let $\mathcal{O}(\mathbb{C})$ be the space of holomorphic functions on $\mathbb{C}$. Let $\psi \in \mathrm{C}^{\infty}(\mathbb{C})$ be a positive, $\mathbb{T}^{1}$-invariant function. Let $\mathrm{L}^{2}(\mathbb{C}, \psi)$ be the completion of $\mathrm{C}_{\mathrm{c}}^{\infty}(\mathbb{C})$ to a Hilbert space with respect to the inner product

$$
\left(f_{1}, f_{2}\right)_{\psi}:=\left(\psi f_{1}, \psi f_{2}\right)_{L^{2}(\mathbb{C})} .
$$

Let $\pi$ be the representation of $\mathbb{T}^{1}$ in $L^{2}(\mathbb{C}, \psi)$ given by

$$
(\pi(g) f)(z)=f\left(g^{-1} z\right)
$$

for all $g \in \mathbb{T}^{1}, f \in \mathrm{L}^{2}(\mathbb{C}, \psi)$ and $z \in \mathbb{C}$.

Set

$$
\mathcal{O}_{\mathrm{L}^{2}}(\mathbb{C}, \psi):=\mathcal{O}(\mathbb{C}) \cap \mathrm{L}^{2}(\mathbb{C}, \psi) .
$$

For $k \in \mathbb{Z}_{\geq 0}$, let $e^{k} \in \mathcal{O}(\mathbb{C})$ be the function $z \mapsto z^{k}$. Then for all $k \in \mathbb{Z}_{\geq 0}$ and $z \in \mathbb{C}$,

$$
\pi(\mathrm{g}) \mathrm{e}^{\mathrm{k}}=\mathrm{g}^{-\mathrm{k}} \mathrm{e}^{\mathrm{k}} .
$$

Suppose $\psi$ was chosen so that $e^{k} \in \mathrm{L}^{2}(\mathbb{C}, \psi)$ for all $k$. For example, one can take $\psi(z)=e^{-|z|^{2} / 2}$.

Let $\Omega_{\mathrm{T}_{2}}^{0, *}(\mathbb{C})$ be the Hilbert space of square-integrable forms of type $(0, *)$. Let $\Omega_{\mathrm{L}^{2}}^{0, *}(\mathbb{C}, \psi)$ be the analogous Hilbert space with the inner product weighted by $\psi$ as in (6.5). Set

$$
\operatorname{ker}_{\mathrm{L}^{2}, \psi}\left(\bar{\partial}+\bar{\partial}^{*}\right)^{ \pm}:=\operatorname{ker}\left(\bar{\partial}+\bar{\partial}^{*}\right)^{ \pm} \cap \Omega_{\mathrm{L}^{2}}^{0, *}(\mathbb{C}, \psi) .
$$

We can realise the distribution $\Theta$ given by the $g$-indices of $\bar{\partial}+\bar{\partial}^{*}$ in terms of the representation of $\mathbb{T}^{1}$ in this space.

Proposition 6.5. The restriction of the representation $\pi$ of $\mathbb{T}^{1}$ to $\operatorname{ker}_{\mathrm{L}^{2}, \psi}\left(\bar{\partial}+\bar{\partial}^{*}\right)^{ \pm}$ has a distributional character $\chi^{ \pm}$, and we have

$$
\Theta=\chi^{+}-\chi^{-} \in \mathcal{D}^{\prime}\left(\mathbb{T}^{1}\right) \text {. }
$$

Proof. First note that

$$
\begin{aligned}
& \operatorname{ker}\left(\bar{\partial}+\bar{\partial}^{*}\right)^{+}=\mathcal{O}(\mathbb{C}) ; \\
& \operatorname{ker}\left(\bar{\partial}+\bar{\partial}^{*}\right)^{-}=0 .
\end{aligned}
$$

So we only need to consider the even part of $\operatorname{ker}_{L^{2}, \psi}\left(\bar{\partial}+\bar{\partial}^{*}\right)$, which equals

$$
\operatorname{ker}_{\mathrm{L}^{2}, \psi}\left(\bar{\partial}+\bar{\partial}^{*}\right)^{+}=\mathcal{O}_{\mathrm{L}^{2}}(\mathbb{C}, \psi) .
$$

The functions $\left\{e^{k}\right\}_{k \geq 0}$ form an orthogonal basis of $\mathcal{O}_{\mathrm{L}^{2}}(\mathbb{C}, \psi)$. By (6.6), the character of the representation $\pi$ on the space (6.7) equals the series (6.4), which converges to $\Theta$. 
Remark 6.6. The $L^{2}(\mathbb{C}, \psi)$-kernel of $\bar{\partial}+\bar{\partial}^{*}$ can be identified as the $L^{2}-$ kernel of a deformed operator. For example, let $\psi(z)=e^{-|z|^{2} / 2}$. Recall that $\bar{\partial}+\bar{\partial}^{*}$ is an operator on $\Omega^{0, *}(\mathbb{C})$, given by

$$
\bar{\partial}+\bar{\partial}^{*}=c(d z) \frac{\partial}{\partial z}+c(d \bar{z}) \frac{\partial}{\partial \bar{z}},
$$

where now $c(d \bar{z})=\frac{1}{\sqrt{2}} \operatorname{ext}(d \bar{z})$ and $c(d z)=-\frac{1}{\sqrt{2}} \operatorname{int}(d z)$. (See [8, Section 3.6].) Set

$$
\mathrm{b}:=\frac{1}{2} z \mathrm{c}(\mathrm{d} \bar{z}) \text {. }
$$

Then $\mathrm{b}^{*}=-\frac{1}{2} \bar{z} \mathrm{c}(\mathrm{d} z)$. We have the deformed operator

$$
\begin{aligned}
\bar{\partial}+b & =c(d \bar{z})\left(\frac{\partial}{\partial \bar{z}}+\frac{z}{2}\right): \Omega^{0,0}(\mathbb{C}) \rightarrow \Omega^{0,1}(\mathbb{C}) \\
(\bar{\partial}+b)^{*} & =c(d z)\left(\frac{\partial}{\partial z}-\frac{\bar{z}}{2}\right): \Omega^{0,1}(\mathbb{C}) \rightarrow \Omega^{0,0}(\mathbb{C}) .
\end{aligned}
$$

The operator $\mathrm{U}: \Omega^{0, *}(\mathbb{C}) \rightarrow \Omega^{0, *}(\mathbb{C}, \psi)$ given by $\mathrm{U}(\alpha)=\psi^{-1} \alpha$ is a unitary isomorphism. We have

$$
\bar{\partial} u(f)=\bar{\partial}\left(\psi^{-1} f\right)=\psi^{-1}\left(\bar{\partial}+\frac{z}{2}\right) f=u\left(\left(\bar{\partial}+\frac{z}{2}\right) f\right) .
$$

Similarly, $\mathrm{U}$ intertwines $\bar{\partial}^{*}$ and $(\bar{\partial}+b)^{*}$. It then follows that

$$
\begin{aligned}
& \operatorname{ker}_{\mathrm{L}^{2}}(\bar{\partial}+\mathrm{b}) \cong \operatorname{ker}_{\mathrm{L}^{2}, \psi}(\bar{\partial}) ; \\
& \operatorname{ker}_{\mathrm{L}^{2}}(\bar{\partial}+\mathrm{b})^{*} \cong \operatorname{ker}_{\mathrm{L}^{2}, \psi}\left(\bar{\partial}^{*}\right)=0 .
\end{aligned}
$$

\subsection{The circle acting on the two-sphere}

As in Subsection 6.3, we consider the circle group $\mathbb{T}^{1}$, this time acting by rotations on the two-sphere $S^{2}$. In this compact setting, the usual index theory, and the Atiyah-Segal-Singer theorem apply. But we can use the g-index to decompose indices in this case.

We embed $\mathbb{T}^{1} \cong \mathrm{SO}(2)$ into $\mathrm{SO}(3)$ in the top-left corner. Then $\mathrm{S}^{2}=\mathrm{SO}(3) / \mathbb{T}^{1}$. Identifying this space with $\mathbb{P}^{1}(\mathbb{C})$, we obtain a complex structure on it. Fix $n \in \mathbb{Z}_{\geq 0}$. Let $\mathbb{C}_{n}$ be the space of complex numbers, on which $\mathbb{T}^{1}$ acts by

$$
g \cdot z=g^{n} z
$$

for $g \in \mathbb{T}^{1}$ and $z \in \mathbb{C}_{n}$. We have the line bundle

$$
\mathrm{L}_{\mathrm{n}}:=\mathrm{SO}(3) \times_{\mathbb{T}^{1}} \mathbb{C}_{\mathrm{n}} \rightarrow \mathrm{S}^{2} .
$$

Let $\bar{\partial}_{n}+\bar{\partial}_{n}^{*}$ be the Dolbeault-Dirac operator on $S^{2}$, coupled to $L_{n}$. Since $S^{2}$ is compact, we have the equivariant index

$$
\operatorname{index}_{\mathrm{SO}(3)}\left(\bar{\partial}_{n}+\bar{\partial}_{n}^{*}\right) \in \mathrm{R}(\mathrm{SO}(3)) .
$$


By the Borel-Weil-Bott theorem, this index is the irreducible representation $V_{n}$ of $\mathrm{SO}(3)$ with highest weight $n$ (with respect to the positive root corresponding to the identification of $S^{2}$ with $\mathbb{P}^{1}(\mathbb{C})$ ).

Fix $g \in \mathbb{T}^{1}$ with dense powers. By the Atiyah-Segal-Singer theorem, or Corollary 6.3, the character of $V_{n}$ evaluated at $g$ equals

$$
\operatorname{index}_{\mathbb{T}^{1}}\left(\bar{\partial}_{n}+\bar{\partial}_{n}^{*}\right)(g)=\frac{g^{n}}{1-g^{-1}}+\frac{g^{-n}}{1-g} .
$$

The two terms on the right hand side correspond to the two fixed points of the action by $\mathbb{T}^{1}$. This expression can be rewritten as the finite sum

$$
\sum_{j=0}^{2 n} g^{j-n} .
$$

This is the usual decomposition of $\left.V_{n}\right|_{\mathbb{T}^{1}}$ into irreducible representations of $\mathbb{T}^{1}$.

So far, we have done nothing new in this example. But let $\bar{\partial}^{\mathbb{C}}+\left(\bar{\partial}^{\mathbb{C}}\right)^{*}$ be the Dolbeault-Dirac operator on $\mathbb{C}$. Then the linearisation theorem, Corollary 6.4 . implies that

$$
\operatorname{index}_{\mathbb{T}^{1}}\left(\bar{\partial}_{\mathfrak{n}}+\bar{\partial}_{n}^{*}\right)(g)=\operatorname{index}_{g}\left(\bar{\partial}^{\mathbb{C}}+\left(\bar{\partial}^{\mathbb{C}}\right)^{*}\right) g^{n}+\operatorname{index}_{g^{-1}}\left(\bar{\partial}^{\mathbb{C}}+\left(\bar{\partial}^{\mathbb{C}}\right)^{*}\right) g^{-n} \text {. }
$$

As we saw in Subsection 6.3, Corollary 6.3 implies that

$$
\operatorname{index}_{g}\left(\bar{\partial}^{\mathbb{C}}+\left(\bar{\partial}^{\mathbb{C}}\right)^{*}\right)=\frac{1}{1-g^{-1}},
$$

and similarly with $g$ replaced by $\mathrm{g}^{-1}$. This agrees with (6.8). Using Proposition 6.5, we can realise the latter index as the character of the representation of $\mathbb{T}^{1}$ in

$$
\operatorname{ker}_{\mathrm{L}^{2}, \psi}\left(\bar{\partial}+\bar{\partial}^{*}\right)^{+}
$$

with $\psi$ as in Subsection 6.3

\subsection{Discrete series characters}

Let $\mathrm{G}$ be a connected, semisimple Lie group. Let $\mathrm{T}<\mathrm{G}$ be a maximal torus, and suppose it is a Cartan subgroup of $G$, i.e. $G$ has discrete series representations. Let $\mathrm{K}<\mathrm{G}$ be a maximal compact subgroup containing $\mathrm{T}$. We denote the normalisers of $T$ in $G$ and $K$ by $N_{G}(T)$ and $N_{K}(T)$, respectively.

Lemma 6.7. The fixed point set of the action by $T$ on $G / T$ is $N_{K}(T) / T$, the Weyl group $W_{c}$ of $\left(\mathfrak{k}_{\mathbb{C}}, \mathfrak{t}_{\mathbb{C}}\right)$.

Proof. Since

$$
(\mathrm{G} / \mathrm{T})^{\mathrm{T}}=\mathrm{N}_{\mathrm{G}}(\mathrm{T}) / \mathrm{T}
$$

it is enough to show that

$$
N_{G}(T)=N_{K}(T)
$$


To prove this, let $\mathfrak{g}=\mathfrak{p} \oplus \mathfrak{k}$ be the Cartan decomposition of $\mathfrak{g}$. Suppose $X \in \mathfrak{p}$, such that $\exp (t X) \in N_{G}(T)$ for all $t \in \mathbb{R}$. Then for all $H \in t$,

$$
\exp (\mathrm{tX}) \exp (\mathrm{H}) \exp (-\mathrm{tX})=\exp (\operatorname{Ad}(\exp (\mathrm{tX})) \mathrm{H}) \in \mathrm{T} .
$$

So $[X, H] \in \mathfrak{t}$. Because $X \in \mathfrak{p}$ and $H \in \mathfrak{t} \subset \mathfrak{k}$, we have $[X, H] \in \mathfrak{p}$. Hence $[X, H]=0$. Since $t$ is maximal commutative, we find that $X \in \mathfrak{t}$, so that $X=0$. Therefore, an element $Y \in \mathfrak{g}$ such that $\exp (\mathrm{t} Y) \in \mathrm{N}_{\mathrm{G}}(\mathrm{T})$ for all $t \in \mathbb{R}$ must lie in $\mathfrak{k}$. Since $\mathrm{G}$ is connected, the claim follows.

Example 6.8. If $G=S L(2, \mathbb{R})$, then a strongly elliptic coadjoint orbit of $G$ is equivariantly diffeomorphic to G/T. This is now a hyperbolic plane, on which $\mathrm{T}$ acts by rotations. This action has one fixed point, corresponding to the trivial Weyl group of $\mathrm{K}=\mathrm{T}$.

Let $\lambda \in i t^{*}$ be regular (in the sense that $(\alpha, \lambda) \neq 0$ for all roots $\alpha$, for a Weyl group invariant inner product). Fix a set $R^{+}$of positive roots for $\left(\mathfrak{g}_{\mathbb{C}}, \mathfrak{t}_{\mathbb{C}}\right)$ by defining a root $\alpha$ to be positive if $(\alpha, \lambda)>0$. Let $\rho$ be half the sum of the positive roots. The choice of positive roots determines a G-invariant complex structure on the manifold $\mathrm{G} / \mathrm{T}$, defined by

$$
\mathrm{T}_{e}^{\mathrm{O}, 1}(\mathrm{G} / \mathrm{T})=(\mathfrak{g} / \mathfrak{t})^{0,1}:=\bigoplus_{\alpha \in \mathrm{R}^{+}}\left(\mathfrak{g}_{\mathbb{C}}\right)_{-\alpha} .
$$

Suppose $\lambda+\rho$ is an integral weight. Then $\lambda-\rho$ is integral as well, and we have the holomorphic line bundle

$$
\mathrm{L}_{\lambda-\rho}:=\mathrm{G} \times_{\mathrm{T}} \mathbb{C}_{\lambda-\rho} \rightarrow \mathrm{G} / \mathrm{T},
$$

where $T$ acts on $\mathbb{C}_{\lambda-\rho}:=\mathbb{C}$ via the weight $e^{\lambda-\rho}$. Let

$$
\bar{\partial}_{L_{\lambda-\rho}}+\bar{\partial}_{L_{\lambda-\rho}}^{*}
$$

be the Dolbeault-Dirac operator on $G / T$, coupled to $L_{\lambda-\rho}$. Let $g \in T$ be such that the powers of $g$ are dense in $T$. (Then in particular, $g$ is a regular element.)

Let $\Theta_{\lambda}$ be the distributional character of the discrete series representation of $\mathrm{G}$ with infinitesimal character $\lambda$.

Proposition 6.9. One has

$$
\operatorname{index}_{g}\left(\bar{\partial}_{L_{\lambda-\rho}}+\bar{\partial}_{L_{\lambda-\rho}}^{*}\right)=(-1)^{\frac{\operatorname{dim} G / K}{2}} \Theta_{\lambda}(g) .
$$

Proof. The proof is analogous to Atiyah and Bott's derivation of the Weyl character formula from their fixed point theorem in [2. Section 5]. By Corollary 6.3 and Lemma 6.7 we have

$$
\operatorname{index}_{g}\left(\bar{\partial}_{L_{\lambda-\rho}}+\bar{\partial}_{L_{\lambda-\rho}}^{*}\right)=\sum_{a T \in N_{K}(T) / T} \frac{e^{\lambda-\rho}\left(a^{-1} g a\right)}{\operatorname{det}\left(1-\mathrm{Ad}_{\mathfrak{g} / \mathfrak{t}}^{0,1}\left(a^{-1} g a\right)\right)} .
$$


Here $\operatorname{Ad}_{\mathfrak{g} / \mathfrak{t}}^{0,1}: T \rightarrow G L\left((\mathfrak{g} / \mathfrak{t})^{0,1}\right)$ is induced by the adjoint representation. Because of (6.9), we have

$$
\operatorname{det}\left(1-\operatorname{Ad}_{\mathfrak{g} / \mathfrak{t}}^{0,1}\left(a^{-1} g a\right)\right)=\prod_{\alpha \in R^{+}}\left(1-e^{-\alpha}\left(a^{-1} g a\right)\right) .
$$

Since in the identification $N_{K}(T) / T=W_{c}$, the normaliser $N_{K}(T)$ acts on $i^{*}$ via the coadjoint action, we find that (6.10) equals

$$
\sum_{w \in W_{c}} \frac{e^{w \cdot(\lambda-\rho)}}{\prod_{\alpha \in R^{+}}\left(1-e^{-w \cdot \alpha}\right)}(g) .
$$

Consider the Weyl denominator

$$
\Delta:=e^{\rho} \prod_{\alpha \in R^{+}}\left(1-e^{-\alpha}\right) .
$$

One has for all $w \in W_{c}$

$$
w \cdot \Delta:=e^{w \cdot \rho} \prod_{\alpha \in R^{+}}\left(1-e^{-w \cdot \alpha}\right)=\varepsilon(w) \Delta,
$$

where $\varepsilon(w)=\operatorname{det} w$ is the sign of $w$. Hence we find that (6.11) equals

$$
\frac{\sum_{w \in W_{c}} \varepsilon(w) e^{w \cdot \lambda}}{\Delta}(\mathrm{g}) .
$$

(This expression still makes sense if $\rho$ is not an integral weight.) By HarishChandra's character formula for the discrete series (see [22, Theorem 16] or [27. Theorem 12.7]), this equals $(-1)^{\frac{\operatorname{dim} G / K}{2}} \Theta_{\lambda}(g)$.

Note that Proposition 6.9 only relates the value of the character $\Theta_{\lambda}$ at $g$ to the $g$-index of $\bar{\partial}_{L_{\lambda-\rho}}+\bar{\partial}_{L_{\lambda-\rho}}^{*}$ if $g$ is a regular element of a maximal torus. Such elements form an open subset of $\mathrm{G}$, and characters are not determined by their restrictions to this set. However, we can still use Proposition 6.9 to give a description of the g-index in terms of the kernel of $\left(\bar{\partial}_{L_{\lambda-\rho}}+\bar{\partial}_{L_{\lambda-\rho}}^{*}\right)$.

Proposition 6.10. Suppose $\mathrm{G}$ is a linear group. Then the representation of $\mathrm{G}$ in the $\mathrm{L}^{2}-$ kernel of $\left(\bar{\partial}_{\mathrm{L}_{\lambda-\rho}}+\bar{\partial}_{\mathrm{L}_{\lambda-\rho}}^{*}\right)^{ \pm}$has a distributional character $\Theta^{ \pm}$that can be evaluated at $\mathrm{g}$, and one has

$$
\operatorname{index}_{g}\left(\bar{\partial}_{L_{\lambda-\rho}}+\bar{\partial}_{L_{\lambda-\rho}^{*}}^{*}\right)=\Theta^{+}(g)-\Theta^{-}(g) .
$$

Proof. This follows from Proposition 6.9 and Schmid's realisation of the discrete series in the $L^{2}$-Dolbeault cohomology of $G / T$ with values in $L_{\lambda-\rho}$, in [33. Theorem 1.5]. Schmid's result implies that space

$$
\operatorname{ker}_{\mathrm{L}^{2}}\left(\bar{\partial}_{\mathrm{L}_{\lambda-\rho}}+\bar{\partial}_{\mathrm{L}_{\lambda-\rho}}^{*}\right)^{ \pm}
$$

equals zero if $\pm=-(-1)^{\frac{\mathrm{dim} G / K}{2}}$, and the representation of $\mathrm{G}$ in this space is the discrete series representation with infinitesimal character $\lambda$ if $\pm=(-1)^{\frac{\operatorname{dim} \mathrm{G} / \mathrm{K}}{2}}$. 
(The integer $k$ in Schmid's result now equals $\operatorname{dim}(G / K) / 2$, and his $\lambda$ is our $\lambda-\rho$.) Hence

$$
\Theta^{+}-\Theta^{-}=(-1)^{\frac{\operatorname{dim} G / K}{2}} \Theta_{\lambda} .
$$

So the claim follows from Proposition 6.9.

\subsection{Fredholm operators}

For Fredholm operators, it is a natural question how the g-index of such an operator is related to the traces of $\mathrm{g}$ acting on even and odd parts of its kernel. This depends on the behaviour of the operator 'towards infinity'. To make this more explicit, let $\mathrm{M}^{+}$be the one-point compactification of $M$. The point at infinity is fixed by $\mathrm{g}$. Let $\mathrm{U}, \mathrm{V} \subset \mathrm{M}$ be as in Subsection 2.2 Let $\mathrm{U}^{\prime}, \mathrm{V}^{\prime} \subset$ $\mathrm{M}^{+}$be g-invariant neighbourhoods of the point at infinity, such that $\overline{\mathrm{U}^{\prime}} \subset \mathrm{V}^{\prime}$, and $\mathrm{V} \cap \mathrm{V}^{\prime}=\emptyset$. Then $\mathrm{U} \sqcup \mathrm{U}^{\prime}$ and $\mathrm{V} \sqcup \mathrm{V}^{\prime}$ are neighbourhoods of $\left(\mathrm{M}^{+}\right)^{g}$ as in (2.2). Lemma 2.4 therefore implies that for any $\sigma$-unital G-C* algebra $A$, the following diagram commutes:

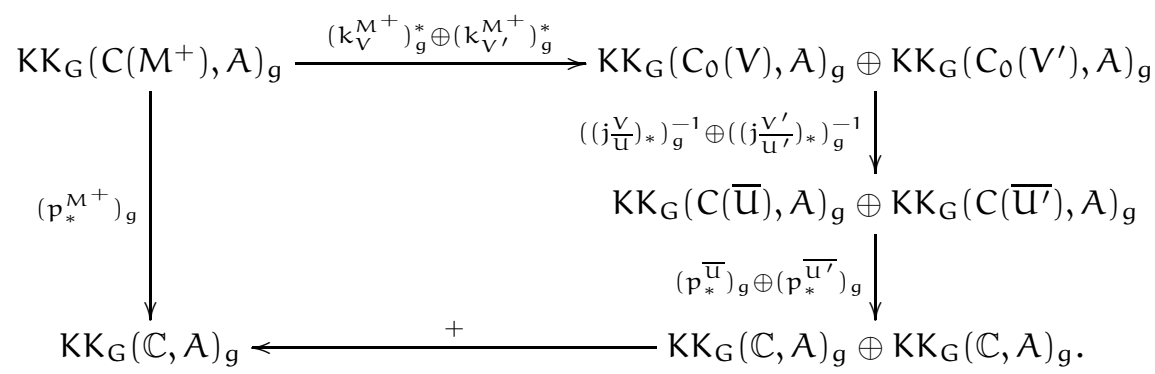

Indeed, since $M^{+}$is compact, one can apply Lemma 2.4 to the pairs of neighbourhoods $\mathrm{U} \sqcup \mathrm{U}^{\prime} \subset \mathrm{V} \sqcup \mathrm{V}^{\prime}$ and $\mathrm{M}^{+} \subset \mathrm{M}^{+}$of $\left(\mathrm{M}^{+}\right)^{\mathrm{g}}$.

Now suppose that $\left(D^{2}+1\right)^{-1}$ is a compact operator. Then $F:=\frac{D}{\sqrt{D^{2}+1}}$ is Fredholm, so $\operatorname{ker}_{\mathrm{L}^{2}}(\mathrm{D})$ is finite-dimensional. Let the representation $\pi_{\mathrm{M}^{+}}: \mathrm{C}\left(\mathrm{M}^{+}\right) \rightarrow$ $\mathcal{B}\left(\mathrm{L}^{2}(\mathrm{E})\right)$ be defined by

$$
\pi_{M^{+}}(f+z)=\pi_{M}(f)+z,
$$

for $f \in C_{0}(M)$ and $z \in \mathbb{C}$. Then the triple $\left(L^{2}(E), F, \pi_{M^{+}}\right)$is a Kasparov $\left(\mathrm{C}\left(\mathrm{M}^{+}\right), \mathbb{C}\right)$-module. Let

$$
\mathrm{M}^{+}[\mathrm{D}] \in \mathrm{KK}_{\mathrm{G}}\left(\mathrm{M}^{+}, \mathrm{pt}\right)
$$

be its class. In this case, we will write index ${ }_{g}^{\infty}(D)$ for a version of the $g$-index of $D$ that captures the behaviour of $D$ at infinity:

$$
\operatorname{index}_{g}^{\infty}(D):=\left(\operatorname{ev}_{g}\right) \circ\left(p_{*}^{\overline{U^{\prime}}}\right)_{g} \circ\left(\left(j \frac{V^{\prime}}{\bar{U}^{\prime}}\right)_{*}\right)_{g}^{-1} \circ\left(k_{V^{\prime}}^{M^{+}}\right)_{g}^{*}\left(M^{+}[D]_{g}\right) .
$$

Proposition 6.11. If $\left(\mathrm{D}^{2}+1\right)^{-1}$ is compact, then

$$
\operatorname{Tr}\left(g \text { on } \operatorname{ker}_{\mathrm{L}^{2}}\left(\mathrm{D}^{+}\right)\right)-\operatorname{Tr}\left(\mathrm{g} \text { on } \operatorname{ker}_{\mathrm{L}^{2}}\left(\mathrm{D}^{-}\right)\right)=\operatorname{index}_{\mathbf{g}}(\mathrm{D})+\operatorname{index}_{\mathbf{g}}^{\infty}(\mathrm{D}) \text {. }
$$


Proof. By commutativity of (6.12), with $A=\mathbb{C}$, we have

$$
\left(\mathrm{ev}_{\mathrm{g}}\right)_{\mathrm{g}} \circ\left(\mathrm{p}_{*}^{\mathrm{M}^{+}}\right)_{\mathrm{g}}[\mathrm{D}]=\operatorname{index}_{\mathrm{g}}(\mathrm{D})+\operatorname{index}_{\mathrm{g}}^{\infty}(\mathrm{D}) .
$$

Now

$$
\mathrm{p}_{*}^{\mathrm{M}^{+}}[\mathrm{D}]=\left[\mathrm{L}^{2}(\mathrm{E}), \mathrm{F}\right]=[\operatorname{ker} \mathrm{F}, 0] \quad \in \mathrm{KK}_{\mathrm{G}}(\mathrm{pt}, \mathrm{pt}),
$$

so the left hand sides of (6.16) and (6.17) are equal.

In concrete situations, knowledge of index $\operatorname{g}_{\mathrm{g}}^{\infty}(\mathrm{D})$ then allows one to use the fixed point formula in Theorem 2.9 to compute the left hand side of (6.16).

This can be made more explicit in a situation relevant to the treatment of Callias-type deformations of Dirac operators in the context of KK-theory in [15, 28]. Suppose that $\Phi \in \operatorname{End}(E)^{G}$ is an odd, self-adjoint vector bundle endomorphism. Suppose that $\Phi^{2}-1_{\mathrm{E}}$ tends to zero at infinity, so that it is a compact operator on $\Gamma_{0}(E)$. Then $\left(\Gamma_{0}(E), \Phi, \pi_{M^{+}}\right)$is an equivariant Kasparov $\left(\mathrm{C}\left(\mathrm{M}^{+}\right), \mathrm{C}_{0}(\mathrm{M})\right)$-cycle. Let $[\Phi] \in \mathrm{KK}_{\mathrm{G}}\left(\mathrm{M}^{+}, M\right)$ be its class. Now we do not assume that $\left(D^{2}+1\right)^{-1}$ itself is compact, but that

$$
\left[\mathrm{D}_{\Phi}\right]:=[\Phi] \otimes_{\mathrm{M}}[\mathrm{D}] \quad \in \mathrm{KK}_{\mathrm{G}}\left(\mathrm{M}^{+}, \mathrm{pt}\right)
$$

is the class of an elliptic operator $D_{\Phi}$ as in (6.14). Then $\left(D_{\Phi}^{2}+1\right)^{-1}$ is compact. (The idea is that $\mathrm{D}_{\Phi}=\mathrm{D}+\Phi$ if $\mathrm{D} \Phi+\Phi \mathrm{D}$ is sufficiently small; Compare this with [15, Proposition 2.18].) By functoriality of the Kasparov product, we have for $\mathrm{U}^{\prime}, \mathrm{V}^{\prime} \subset \mathrm{M}$ as above,

$$
\operatorname{index}_{g}^{\infty}\left(D_{\Phi}\right)=\left(e_{g}\right)_{g}\left(\left(\left(p_{*}^{\overline{u^{\prime}}}\right)_{g} \circ\left(\left(j \frac{V^{\prime}}{U^{\prime}}\right)_{*}\right)_{g}^{-1} \circ\left(k_{V^{\prime}}^{M^{+}}\right)_{g}^{*}[\Phi]_{g}\right) \otimes_{M}[D]_{g}\right) \text {. }
$$

This expression has the advantage that $\Phi$ is a vector bundle endomorphism, which makes (6.18) easier to evaluate than (6.15). In particular, if $\Phi^{2}=1_{\mathrm{E}}$ on $\mathrm{V}^{\prime} \cap \mathrm{M}$, then $\left(\mathrm{k}_{V^{\prime}}^{\mathrm{M}^{+}}\right)^{*}[\Phi]=0$. In that case, Theorem 2.9 and Proposition 6.11 imply that

$\operatorname{Tr}\left(g\right.$ on $\left.\operatorname{ker}_{\mathrm{L}^{2}}\left(\mathrm{D}_{\Phi}^{+}\right)\right)-\operatorname{Tr}\left(\mathrm{g}\right.$ on $\left.\operatorname{ker}_{\mathrm{L}^{2}}\left(\mathrm{D}_{\Phi}^{-}\right)\right)=\int_{\mathrm{TM}^{g}} \frac{\operatorname{ch}\left(\left[\left.\sigma_{\mathrm{D}_{\Phi}}\right|_{\mathrm{TM}^{g}}\right](\mathrm{g})\right) \operatorname{Todd}\left(\mathrm{TM}^{\mathrm{g}} \otimes \mathbb{C}\right)}{\operatorname{ch}\left(\left[\bigwedge \mathrm{N}_{\mathbb{C}}\right](\mathrm{g})\right)}$.

Example 6.12. Let $M=\mathbb{C}^{n}$, and let $\mathrm{g}$ be the diagonal action by $n$ nontrivial elements of $U(1)$. Then $M^{g}=\{0\}$, and $N=\mathbb{C}^{n}$. Let $\beta_{\mathbb{C}^{n}} \in K K_{G}\left(p t, \mathbb{C}^{2 n}\right)$ be the Bott element as in Definition 4.6. Now the class $\left[D_{1}\right] \in K_{\mathrm{G}}\left(\mathbb{C}^{2 n}, \mathrm{pt}\right)$ as in (4.8) is the Dolbeault class of $\mathbb{C}^{2 n}$. The Kasparov product

$$
\beta_{\mathbb{C}^{n}} \otimes_{\mathbb{C}^{2 n}}\left[D_{1}\right] \quad \in K K_{G}(p t, p t)
$$

is represented by the elliptic operator $D_{B}:=B \otimes 1+1 \otimes D_{1}$ as in (4.12). Hence $\left(D_{B}^{2}+1\right)^{-1}$ is a compact operator. In the proof of Lemma 4.9, we saw that the $\mathrm{L}^{2}$-kernel of $\mathrm{D}_{\mathrm{B}}$ is spanned by the $\mathrm{g}$-invariant function (4.14). So

$$
\operatorname{Tr}\left(\text { g on } \operatorname{ker}_{\mathrm{L}^{2}}\left(\mathrm{D}_{\mathrm{B}}^{+}\right)\right)-\operatorname{Tr}\left(\mathrm{g} \text { on } \operatorname{ker}_{\mathrm{L}^{2}}\left(\mathrm{D}_{\mathrm{B}}^{-}\right)\right)=1 .
$$


On the other hand, let $b \in C^{\infty}(\mathbb{R})$ be an odd function, with values in $[-1,1]$, such that $b(x)=1$ for all $x \geq 1$. If we replace $B\left(1+B^{2}\right)^{-1 / 2}$ by $b(B)$ in (4.6), then the resulting class in $\mathrm{KK}_{\mathrm{G}}\left(\mathrm{pt}, \mathbb{C}^{2 \mathrm{n}}\right)$ is the same class $\beta_{\mathbb{C}^{n}}$. But with this normalisation function, we have $b(B)^{2}=1$ outside the unit ball in $\mathbb{C}^{n}$. So

$$
\operatorname{index}_{g}^{\infty}\left(D_{B}\right)=0 \text {. }
$$

Finally, by Corollary 6.3, with $F=\wedge \widetilde{N}_{\mathbb{C}}=\wedge \mathbb{C}^{2 n}$, we have

$$
\operatorname{index}_{\mathrm{g}}\left(\mathrm{D}_{\mathrm{B}}\right)=1 \text {. }
$$

The equalities (6.20), (6.21) and (6.22) illustrate Proposition 6.11) in this case.

Example 6.13. In the setting of Theorem 2.10, the index pairing $[F] \otimes_{M}[D] \in$ $\mathrm{KK}_{\mathrm{G}}(\mathrm{pt}, \mathrm{pt})$ is represented by a Fredholm operator $\mathrm{D}_{\mathrm{F}}$. Analogously to (6.18), we have index ${ }_{g}^{\infty}\left(D_{F}\right)=0$, so that Proposition 6.11 and Theorem 2.9 yield an expression for $\operatorname{Tr}\left(g\right.$ on $\left.\operatorname{ker}_{\mathrm{L}^{2}}\left(\mathrm{D}_{\mathrm{F}}^{+}\right)\right)-\operatorname{Tr}\left(\mathrm{g}\right.$ on $\left.\operatorname{ker}_{\mathrm{L}^{2}}\left(\mathrm{D}_{\mathrm{F}}^{-}\right)\right)$. But in this setting, the same expression follows directly from Theorem 2.10

See Remark 7.6 for the construction of a Fredholm operator $D_{f v}$ as a deformation of any elliptic operator D, with index $\operatorname{s}_{g}^{\infty}\left(D_{f v}\right)=0$.

\subsection{Braverman's index}

Suppose $X \in \mathfrak{g}$ such that $g=\exp X$. Let $X^{M}$ be the vector field on $M$ defined by X. Suppose D is a Dirac-type operator, and consider the deformed operator

$$
\mathrm{D}_{\mathrm{X}}^{\mathrm{f}}:=\mathrm{D}+\sqrt{-1} \mathrm{fc}\left(\mathrm{X}^{\mathrm{M}}\right) \text {. }
$$

Here $f \in C^{\infty}(M)^{G}$, and $c: T M \rightarrow \operatorname{End}(E)$ is a given Clifford action, used to define the Dirac operator D. Braverman obtained a fixed point theorem for such operators, in [12, Theorem 7.5]. This implies that the g-index equals Braverman's index in this case.

Corollary 6.14. If $\mathrm{f}$ is admissible (Definition 2.6 in [12]), then the representation of $\mathrm{G}$ in $\operatorname{ker}_{\mathrm{L}^{2}}\left(\mathrm{D}_{\mathrm{X}}^{\mathrm{f}}\right)^{ \pm}$has a character $\chi^{ \pm}$that can be evaluated at $\mathrm{g}$, and one has

$$
\operatorname{index}_{g}(D)=\chi^{+}(g)-\chi^{-}(g) .
$$

Proof. The fixed point formula for index ${ }_{g}(\mathrm{D})$ in Theorem 2.9 is precisely the evaluation at $g$ of the right hand side of the formula in [12, Theorem 7.5]. (This equality also shows that $\operatorname{ker}_{\mathrm{L}^{2}}\left(\mathrm{D}_{\mathrm{X}}^{\mathrm{f}}\right)$ has a character that can be evaluated at $\mathrm{g}$.)

Remark 6.15. In the above construction, the element $X \in \mathfrak{g}$, which represents the taming map used in [12], depends on the group element $\mathrm{g}$. So the $\mathrm{g}$-index of $\mathrm{D}$ is not the character of the Braverman index of $\mathrm{D}$ deformed by a single taming map, but the taming map depends on $\mathrm{g}$. 


\subsection{A relative index theorem}

In Theorem 4.18 of [20], Gromov and Lawson obtain a relative index formula for pairs of elliptic operators that coincide outside compact sets. (See Theorem 2.18 in [14] for a version for Callias-type operators.) There is an analogue of this result for the $g$-index.

For $j=0,1$, let $M_{j}$ be a manifold with the same structure and properties as $M$. Let $E_{j} \rightarrow M_{j}$ be a vector bundle like $E \rightarrow M$, and let $D_{j}$ be an operator on $E_{j}$ like $D$ on $E$. Suppose there are relatively compact neighbourhoods $U_{j}$ of $M_{j}^{g}$ outside of which $M_{j}, E_{j}$ and $D_{j}$ can be identified. As on page 38 of [20], we compactify $M_{j}$ to a manifold $\tilde{M}_{j}$, by taking a neighbourhood $V_{j}$ of $\mathrm{U}_{j}$, and attaching a compact manifold $X$ to it. Since $M_{0} \backslash V_{0}=M_{1} \backslash V_{1}$, the same manifold $X$ can be used for $j=0,1$. Extend the vector bundles $E_{j}$ and the operators $D_{j}$ to vector bundles $\tilde{E}_{j} \rightarrow \tilde{M}_{j}$ and elliptic operators $\tilde{D}_{j}$ on $\tilde{E}_{j}$. Suppose the map g extends to $\tilde{M}_{j}$ and $\tilde{E}_{j}$, commuting with $\tilde{D}_{j}$.

Proposition 6.16 (Relative index theorem). We have

$$
\operatorname{index}_{g} D_{1}-\operatorname{index}_{g} D_{0}=\operatorname{index}_{G}\left(\tilde{D}_{1}\right)(g)-\operatorname{index}_{G}\left(\tilde{D}_{0}\right)(g) \text {. }
$$

Since the manifolds $\tilde{M}_{j}$ are compact, the indices on the right hand side of this equality are given by the usual equivariant index.

Proof. By the Atiyah-Segal-Singer fixed point theorem (or Theorem 2.9), we have for $j=0,1$,

$$
\begin{aligned}
\operatorname{index}_{G}\left(\tilde{D}_{j}\right)(g)=\int_{T^{\prime}} \frac{\operatorname{ch}\left(\left[\left.\sigma_{\tilde{D}_{j}}\right|_{T_{M}} ^{g}\right](g)\right) \operatorname{Todd}\left(\operatorname{TM}_{j}^{g} \otimes \mathbb{C}\right)}{\operatorname{ch}\left(\left[\bigwedge\left(N_{j}\right)_{\mathbb{C}}\right](g)\right)} \\
\quad+\int_{T^{\prime g}} \frac{\operatorname{ch}\left(\left[\left.\sigma_{\tilde{D}_{j}}\right|_{T X g}\right](g)\right) \operatorname{Todd}\left(\operatorname{TX}^{g} \otimes \mathbb{C}\right)}{\operatorname{ch}\left(\left[\bigwedge\left(N_{X}\right)_{\mathbb{C}}\right](g)\right)} .
\end{aligned}
$$

Here $N_{j} \rightarrow M_{j}^{g}$ and $N_{X} \rightarrow X^{g}$ are normal bundles to fixed point sets. Since $\left.\sigma_{\tilde{D}_{1}}\right|_{T X g}=\left.\sigma_{\tilde{D}_{0}}\right|_{T X g}$, Theorem 2.9 implies the claim.

\subsection{Some geometric consequences}

The vanishing or non-vanishing of the g-index has some geometric consequences in the cases of Hodge-de Rham and Spin-Dirac operators.

Let $D=d+d^{*}: \Omega_{\mathbb{C}}^{\text {even }}(M) \rightarrow \Omega_{\mathbb{C}}^{\text {odd }}(M)$ be the Hodge-de Rham operator on $M$, acting on complex differential forms. The symbol class of this operator is $\left[\tau_{M}^{*} \wedge \mathrm{TM}_{\mathbb{C}}\right]$, whose restriction to $\mathrm{TM}^{\mathrm{g}}$ equals

$$
\left[\left.\sigma_{\mathrm{D}}\right|_{\mathrm{TM}^{\mathrm{g}}}\right]=\left[\tau_{\mathrm{M}^{g}}^{*} \wedge \mathrm{N}_{\mathbb{C}}\right] \otimes\left[\tau_{M^{g}}^{*} \wedge \mathrm{TM}_{\mathbb{C}}^{\mathrm{g}}\right]
$$

Let $\mathrm{D}_{M \mathrm{~g}}$ be the component-wise defined Hodge-de Rham operator on $\mathrm{M}^{\mathrm{g}}$. Then Theorem 2.9 and (6.23) imply that

$$
\operatorname{index}_{g}\left(d+d^{*}\right)=\int_{T_{M} g} \operatorname{ch}\left(\sigma_{D_{M g}}\right) \operatorname{Todd}\left(T^{g} \otimes \mathbb{C}\right)=\operatorname{index}\left(D_{M^{g}}\right)=\chi\left(M^{g}\right),
$$

the Euler characteristic of $M^{9}$. (See also [29, p. 262].) 
Corollary 6.17. If index $\left(\mathrm{d}+\mathrm{d}^{*}\right) \neq 0$, then every g-invariant vector field on $\mathrm{M}$ has a zero on $\mathrm{M}^{\mathrm{g}}$.

Proof. A g-invariant vector field on $M$ restricts to a vector field on $\mathrm{M}^{\mathrm{g}}$. If it does not vanish there, then $\chi\left(M^{9}\right)=0$. So the claim follows from (6.24).

Next, suppose that $M$ is a Spin manifold, and that $g$ lifts to the spinor bundle. Let $\mathrm{D}$ be the Spin-Dirac operator.

Corollary 6.18. If $\mathrm{G}$ is connected, $\mathrm{M}$ is noncompact, and $\operatorname{index}_{\mathrm{g}}(\mathrm{D})=0$, then the one-point compactification $\mathrm{M}^{+}$of $\mathrm{M}$ is not a G-equivariant Spin manifold

Proof. If $\mathrm{M}^{+}$is a G-equivariant Spin manifold, with Dirac operator $\mathrm{D}_{\mathrm{M}^{+}}$, then the vanishing result of Atiyah and Hirzebruch [3] implies that

$$
0=\operatorname{index}_{g}\left(D_{M^{+}}\right)=\operatorname{index}_{g}(D)+a_{\infty} .
$$

Here $a_{\infty}$ is the contribution of the fixed point at infinity, which is nonzero by [2. Theorem 8.35].

\section{A non-localised index formula}

In the compact case, the Kirillov formula is a non-localised expression for the equivariant index of an elliptic operator; see [8, Theorem 8.2]. This can be deduced from the fixed point formula in the compact case. In the case of noncompact manifolds, there is also a non-localised expression for the g-index, Proposition 7.5 below. This follows from Kasparov's index theorem and the properties of the $\mathrm{g}$-symbol class introduced in Subsection 5.4, rather than from Theorem 2.9 .

A potentially interesting feature of this non-localised formula is that it involves the same kind of deformed symbols as the ones used for Dirac operators on symplectic manifolds in [32]. Those deformed symbols are transversally elliptic rather than elliptic. Berline and Vergne obtained a generalisation of the Atiyah-Segal-Singer fixed point formula to transversally elliptic operators or symbols, see [9. Main Theorem 1] and [10, Theorem 20]. This formula involves a distribution on the group. It was pointed out to the authors by Vergne that this formula implies that for the deformed symbols we will consider, at points $\mathrm{g}$ where this distribution is given by a function, it is given by the $\mathrm{g}$-index.

The index of such a deformed symbol was shown to equal the index of a deformed Dirac operator in Theorem 5.5 in [12]. In Theorem 1.5 in [30], this index is proved to be equal to another index of deformed Dirac operators, defined using the Atiyah-Patodi-Singer index on manifolds with boundary. In contrast to [12, 30, 32], the expression for the $g$-index in terms of deformed symbols is independent of the choices made in this deformation. Furthermore, it applies to more general elliptic operators than Dirac operators.

We shall describe the g-symbol class $\sigma_{g}^{\mathrm{D}}$ of Definition [5.5 more explicitly, using a deformed symbol. Let $v$ be a G-invariant vector field on $M$ that does not vanish outside $V$. 
Example 7.1. If $X \in \mathfrak{g}$ such that $g=\exp (X)$, one can take the vector field $v$ induced by $X$. This vector field obviously depends on $g$.

Example 7.2. If $H$ is a compact Lie group acting on $M$, containing $G$, then it can be possible to choose a single vector field $v$ that works for all elements of $H$. Indeed, suppose there is an H-equivariant map $\psi: M \rightarrow \mathfrak{h}$, and consider the Kirwan vector field $v$, defined by

$$
v_{\mathrm{m}}:=\left.\frac{\mathrm{d}}{\mathrm{dt}}\right|_{\mathrm{t}=0} \exp (\mathrm{t} \psi(\mathrm{m})) \cdot \mathrm{m},
$$

for $m \in M$. Suppose this vector field is nonzero outside a compact set. Then $\psi$ is a taming map as in Definition 2.4 in [12]. In this case, the vector field $v$ can be used for any element of $\mathrm{H}$.

Let $\mathrm{f}: \mathrm{V} \rightarrow \mathbb{R}_{\geq 0}$ be a G-invariant continuous function, such that $\mathrm{f}(\mathrm{m})=0$ when $\mathrm{m} \in \mathrm{U}$ and $\lim _{\mathrm{m} \rightarrow \mathrm{m}^{\prime}} \mathrm{f}(\mathrm{m})=\infty$ if $\mathrm{m}^{\prime} \in \partial \mathrm{V}$. Consider the deformed symbol $\sigma_{D, f v} \in \operatorname{End}\left(\tau_{V}^{*}\left(\left.E\right|_{V}\right)\right)$, given by

$$
\sigma_{D, f v}(u):=\sigma_{D}\left(u+f(m) v_{m}\right)
$$

for $m \in V$ and $u \in T_{m} M$. Set

$$
\tilde{\sigma}_{D, f v}:=\frac{\sigma_{D, f v}}{\sqrt{\sigma_{D, f v}^{2}+1}} .
$$

This defines an odd, self-adjoint, bounded operator on the Hilbert $C_{0}(T V)$ module $\Gamma_{0}\left(\tau_{V}^{*}\left(\left.E\right|_{V}\right)\right)$. Furthermore, we have for every vector field $u$ on $M$, and every $m^{\prime} \in \partial V$,

$$
\lim _{m \rightarrow m^{\prime}} \tilde{\sigma}_{D, f v}\left(u_{m}\right)=\operatorname{sgn}\left(\sigma_{D}\left(v_{m^{\prime}}\right)\right) .
$$

We extend $\tilde{\sigma}_{D, f v}$ to a continuous vector bundle endomorphism of $\tau_{M}^{*} E$ by setting

$$
\tilde{\sigma}_{D, f v}(u):=\operatorname{sgn}\left(\sigma_{D}\left(v_{m}\right)\right)
$$

for all $u \in T_{m} M$, where $m \in M \backslash V$. (Since $v_{m} \neq 0$ if $m \in M \backslash V$, this operator is invertible outside $\mathrm{V}$.)

Note that

$$
\tilde{\sigma}_{D, f v}(u)^{2}-1 \rightarrow 0
$$

as $u \rightarrow \infty$ in TM. Indeed, let $m \in M$ and $u \in T_{m} M$ be given. If $m \notin V$, then $v_{m} \neq 0$ and $\tilde{\sigma}_{D, f v}(u)^{2}=1$. And if $m \in V$, then

$$
\tilde{\sigma}_{D, f v}(u)^{2}-1=\left(\sigma_{D}\left(u+f(m) v_{m}\right)^{2}+1\right)^{-1} .
$$

Since D is elliptic and has first order, this goes to zero as $u \rightarrow \infty$ in TV. We therefore find that $\left(\Gamma_{0}\left(\tau_{M}^{*} E\right), \tilde{\sigma}_{D, f v}\right)$ is a $\operatorname{Kasparov}\left(\mathbb{C}, C_{0}(T M)\right)$-cycle. Let

$$
\mathrm{pt}\left[\sigma_{\mathrm{D}, \mathrm{fv}}\right] \in \mathrm{KK}_{\mathrm{G}}(\mathrm{pt}, \mathrm{TM})
$$

be its class, which will be called the deformed symbol class. 
Lemma 7.3. The localisation of the deformed symbol class at $\mathrm{g}$ is $\sigma_{g}^{\mathrm{D}}$, i.e.,

$$
{ }_{\mathrm{pt}}\left[\sigma_{\mathrm{D}, \mathrm{fv}}\right]_{\mathrm{g}}=\sigma_{\mathrm{g}}^{\mathrm{D}} \in \mathrm{KK} K_{\mathrm{G}}(\mathrm{pt}, \mathrm{TM})_{\mathrm{g}} .
$$

Proof. As in Subsection 6.6, let $\mathrm{M}^{+}$be the one-point compactification of $M$. Let $\mathrm{U}, \mathrm{V}, \mathrm{U}^{\prime}, \mathrm{V}^{\prime} \subset \mathrm{M}^{+}$be as in that subsection. Consider the class

$$
M^{+}\left[\sigma_{D, f v}\right]:=\left[\Gamma_{0}\left(\tau_{M}^{*} E\right), \tilde{\sigma}_{D, f v}, \pi_{M^{+}}\right] \in K_{K}\left(M^{+}, T M\right),
$$

where $\pi_{M^{+}}$is as in (6.13). Then by commutativity of (6.12), for $A=C_{0}(T M)$, we have

$$
\begin{aligned}
\mathrm{pt}_{\mathrm{t}}\left[\sigma_{\mathrm{D}, \mathrm{fv}}\right]_{\mathrm{g}}= & \left(\mathrm{p}_{*}^{\mathrm{M}^{+}}\right)_{\mathrm{g}}\left(\mathrm{M}+\left[\sigma_{\mathrm{D}, \mathrm{fv}}\right]\right) \\
= & \left(\mathrm{p}_{*}^{\overline{\mathrm{u}}}\right)_{\mathrm{g}} \circ\left(\left(j \frac{\mathrm{V}}{\mathrm{U}}\right)_{*}\right)_{\mathrm{g}}^{-1} \circ\left(\mathrm{k}_{\mathrm{V}}^{\mathrm{M}^{+}}\right)_{\mathrm{g}}^{*}\left(\mathrm{M}^{+}\left[\sigma_{\mathrm{D}, \mathrm{fv}}\right]_{\mathrm{g}}\right) \\
& \quad+\left(\mathrm{p}_{*}^{\overline{\mathrm{U}^{\prime}}}\right)_{\mathrm{g}} \circ\left(\left(j \frac{\mathrm{V}^{\prime}}{\mathrm{U}^{\prime}}\right)_{*}\right)_{\mathrm{g}}^{-1} \circ\left(\mathrm{k}_{\mathrm{V}^{\prime}}^{\mathrm{M}^{+}}\right)_{\mathrm{g}}^{*}\left(\mathrm{M}^{+}\left[\sigma_{\mathrm{D}, f v}\right]_{\mathrm{g}}\right) .
\end{aligned}
$$

Now since $f=0$ on $V$, we have

$$
\left(k_{V}^{M^{+}}\right)^{*}\left(M^{+}\left[\sigma_{D, f v}\right]\right)=\left(k_{V}^{M}\right)^{*}\left[\sigma_{D}\right] .
$$

So the first term in (7.3) equals $\sigma_{g}^{\mathrm{D}}$. Furthermore,

$$
\left(k_{V^{\prime}}^{M^{+}}\right)^{*}\left(M^{+}\left[\sigma_{D, f v}\right]\right)=\left[\Gamma^{\infty}\left(\left.E\right|_{V^{\prime}}\right), \operatorname{sgn}\left(\sigma_{D}(v)\right), \pi_{V^{\prime}}\right]=0
$$

since this class is represented by a degenerate cycle.

Remark 7.4. Instead of (7.1), we could have used a more general deformed symbol of the form

$$
\sigma_{D, f \Phi}(u):=\sigma_{D}(u)+f(m) \Phi_{m},
$$

for $m \in M, u \in T_{m} M$ and a G-equivariant, fibrewise self-adjoint, odd vector bundle endomorphism $\Phi$ of $E$, which is invertible outside $V$. We have used the natural choice $\Phi=\sigma_{\mathrm{D}}(v)$.

The realisation of the g-symbol class in Lemma 7.3 leads to the following non-local formula for the $\mathrm{g}$-index.

Proposition 7.5 (Non-localised formula for the g-index). The g-index of $\mathrm{D}$ is calculated by

$$
\operatorname{index}_{g}(D)=\left({ }_{p t}\left[\sigma_{D, f v}\right] \otimes_{T M}\left[D_{T M}\right]\right)(g) .
$$

Proof. It follows from Definitions 2.3 and 5.5, and Theorem 4.3, that

$$
\operatorname{index}_{g}(D)=\left(\sigma_{g}^{D} \otimes_{T M}\left[D_{T M}\right]_{g}\right)(g)
$$

The claim therefore follows from Lemma 7.3 
Remark 7.6. Recall that when $M$ is noncompact, $\operatorname{index}_{g}(D)$ is defined using KK-functorial maps. In Proposition 7.5, the class

$$
{ }_{p t}\left[\sigma_{D, f v}\right] \otimes_{T M}\left[D_{T M}\right] \in K K_{G}(p t, p t)
$$

is represented by a Fredholm operator $D_{f v}$, defined in terms of the deformed symbol $\sigma_{\mathrm{D}, \mathrm{fv}}$ and the Dolbeault-Dirac operator D $\mathrm{D}_{\mathrm{TM}}$. Proposition 7.5 states that

$$
\operatorname{index}_{g}(D)=\operatorname{Tr}\left(g \text { on } \operatorname{ker}_{L^{2}}\left(D_{f v}^{+}\right)\right)-\operatorname{Tr}\left(g \text { on } \operatorname{ker}_{L^{2}}\left(D_{f v}^{-}\right)\right) \text {. }
$$

Theorem 2.9 then yields a cohomological expression for the right hand side of (7.5). (Note the analogy with (6.19); we now have index ${ }_{g}^{\infty}\left(D_{f v}\right)=0$.)

\section{References}

[1] N. Anghel, On the index of Callias-type operators, Geom. Funct. Anal. 3 (5) (1993), 431-438.

[2] M.F. Atiyah and R. Bott, A Lefschetz fixed point formula for elliptic complexes. II. Applications. Ann. Math. (2) 88 (1968), 451-491.

[3] M.F. Atiyah, F. Hirzebruch, Spin-manifolds and group actions, 1970 Essays on Topology and Related Topics (Mémoires dédiés Georges de Rham) Springer, New York, 18-28.

[4] M.F. Atiyah and G.B. Segal, The index of elliptic operators: II, Ann. Math. 87 (3) (1968), 531-545.

[5] M.F. Atiyah and I.M. Singer, The index of elliptic operators: I, Ann. Math. 87 (3) (1968), 484-530.

[6] M.F. Atiyah and I.M. Singer, The index of elliptic operators: III, Ann. Math. 87 (3) (1968), 546-604.

[7] P. Baum, A. Connes and N. Higson, Classifying space for proper actions and K-theory of group C*-algebras, Contemp. math. 167 (1994), 241-291.

[8] N. Berline, E. Getzler and M. Vergne, Heat kernels and Dirac operators, Grundlehren der math. Wissenschaften vol. 298, Springer (1992).

[9] N. Berline and M. Vergne, The Chern character of a transversally elliptic symbol and the equivariant index, Invent. Math. 124, (1-3) (1996), 11-49.

[10] N. Berline and M. Vergne, L'indice quivariant des oprateurs transversalement elliptiques, Invent. Math. 124, (1-3) (1996), 51-101.

[11] B. Blackadar, K-theory for operator algebras, second edition, Mathematical Sciences Research Institute Publications, vol. 5, Cambridge University Press (1998).

[12] M. Braverman, Index theorem for equivariant Dirac operators on non-compact manifolds, K-Theory, 27 (1) (2002), 61-101.

[13] M. Braverman, The index theory on non-compact manifolds with proper group action, J. Geom. Phys., 98 (2015), 275-284. 
[14] M. Braverman and P. Shi, Cobordism invariance of the index of Callias-type operators, arXiv:1512.03939

[15] U. Bunke, A K-theoretic relative index theorem and Callias-type Dirac operators, Math. Ann. 303 (2) (1995), 241-279

[16] C. Callias, Axial anomalies and index theorems on open spaces, Comm. Math. Phys. 62 (1978), 213-234.

[17] A.L. Carey, V. Gayral, A. Rennie and F.A. Sukochev, Index theory for locally compact noncommutative geometries, Mem. Amer. Math. Soc. 231 (1085) (2014), vi+130 pp.

[18] I. Dell'Ambrogio, H. Emerson and R. Meyer, An equivariant Lefschetz fixedpoint formula for correspondences, Doc. Math. 19 (2014), 141-194.

[19] H. Emerson, Duality, correspondences and the Lefschetz map in equivariant KK-theory: a survey, Perspectives on noncommutative geometry, Fields Inst. Commun., 61, Amer. Math. Soc., Providence, RI (2011), 4-78.

[20] M. Gromov and H.B. Lawson, Positive scalar curvature and the Dirac operator on complete Riemannian manifolds, Pub. Math. I.H.É.S. 58 (1983), 83-196.

[21] V. Guillemin, V. Ginzburg and Y. Karshon, Moment maps, cobordisms and Hamiltonian group actions, Mathematical surveys and monographs vol. 98, American Mathematical Society (2002).

[22] Harish-Chandra Discrete series of semisimple Lie groups II, explicit determination of the characters, Acta Math. 116 (1966), 1-111.

[23] N. Higson and J. Roe, Analytic K-homology, Oxford mathematical monographs, Oxford University Press (2000).

[24] P. Hochs and V. Mathai, Geometric quantization and families of inner products, Adv. Math. 282 (2015), 362-426.

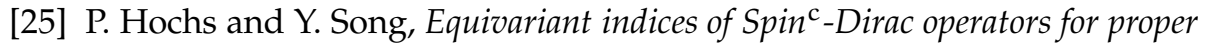
moment maps, arXiv:1503.00801

[26] G.G. Kasparov, K-theoretic index theorems for elliptic and transversally elliptic operators, J. Noncommut. Geom. (to appear), version November 2013.

[27] A. Knapp, Representation theory of semisimple groups, an overview based on examples, Princeton landmarks in math., Princeton Univ. Press (2001).

[28] D. Kucerovsky, A short proof of an index theorem, Proc. Amer. Math. Soc. 129 (12) (2001), 3729-3736.

[29] H.B. Lawson Jr. and M.-L. Michelsohn, Spin Geometry, Princeton University Press (1989).

[30] X. Ma and W. Zhang, Geometric quantization for proper moment maps: the Vergne conjecture, Acta Math., 212 (1) (2014), 11-57.

[31] R.S. Palais, On the existence of slices for actions of non-compact Lie groups, Ann. Math., vol. 73, (2) (1961), 295-323.

[32] P.-É. Paradan. Formal geometric quantization II, Pacific J. Math., 253 (1) (2011), 169-211. 
[33] W. Schmid, L²-cohomology and the discrete series, Ann. Math. 103 (3) (1976), 375-394.

[34] B.-L. Wang and H. Wang, Localized Index and $\mathrm{L}^{2}$-Lefschetz fixed point formula for orbifolds, J. Differential Geom. (to appear), arXiv:1307.2088 